\title{
REDUÇÃO DE ERRO NUMÉRICO NO FILTRO ESTENDIDO DE KALMAN APLICADO À TOMOGRAFIA POR IMPEDÂNCIA ELÉTRICA
}

\author{
Dissertação apresentada à Escola \\ Politécnica da Universidade de São \\ Paulo para obtenção do Título de \\ Mestre em Engenharia.
}

São Paulo 


\section{REDUÇÃO DE ERRO NUMÉRICO NO FILTRO ESTENDIDO DE KALMAN APLICADO À TOMOGRAFIA POR IMPEDÂNCIA ELÉTRICA}

Dissertação apresentada à Escola Politécnica da Universidade de São Paulo para obtenção do Título de Mestre em Engenharia

Área de concentração:

Engenharia Mecânica.

Orientador:

Prof. Dr. Raul González Lima 


\section{FICHA CATALOGRAFICA}

Vanegas Molina, Nelson Antonio

Redução de erro numérico no filtro estendido de Kalman aplicado à tomografia por impedância elétrica. - São Paulo, 2002.

$108 \mathrm{p}$.

Dissertação (Mestrado) - Escola Politécnica da Universidade de São Paulo. Departamento de Engenharia Mecânica.

1. Tomografia por Impedância elétrica 2. Filtro de Kalman 3. Imagens médicas I. Universidade de São Paulo. Escola Politécnica. Departamento de Engenharia Mecânica II.t. 
DE CORAZÓN

A MIS ANCESTROS,

A MI FAMILIA,

A LA VIVENCIA DEL AMOR: CLAUDIA ○ 


\section{INSTANTES}

Si pudiera vivir nuevamente mi vida.

En la próxima trataría de cometer más errores.

No intentaría ser tan perfecto, me relajaría más.

Sería más tonto de lo que he sido, de hecho

tomaría muy pocas cosas con seriedad.

Sería menos higiénico.

Correría más riesgos, haría más viajes, contemplaría más atardeceres, subiría más montañas, nadaría más rios.

Iría a más lugares a donde nunca he ido, comería

más helados y menos habas, tendría más problemas reales y menos imaginarios.

Yo fuí una de esas personas que vivió sensata y prolíficamente cada minuto de su vida; claro que tuve momentos de alegría.

Pero si pudiera volver atrás trataría de tener solamente buenos momentos.

Por si no lo saben, de eso está hecha la vida, sólo de momentos; no te pierdas el ahora.

Yo era uno de esos que nunca iban a ninguna parte sin un termómetro, una bolsa de agua caliente, un paraguas y un paracaídas;

Si pudiera volver a vivir, viajaría más liviano.

Si pudiera volver a vivir comenzaría a andar descalzo a principios de la primavera y seguiría así hasta concluir el otoño.

Daría más vueltas en calesita, contemplaría más atardeceres y jugaría con mas niños, si tuviera otra vez la vida por delante.

Pero ya tengo 85 años y sé que me estoy muriendo.

Jorge Luis Borges 


\section{AGRADECIMENTOS}

Nenhuma obra pode ser culminada sem a ajuda de uma mão amiga. Quero expressar os meus agradecimentos:

- Ao meu orientador, professor Dr. Raul González Lima pela sua acolhida, paciência e colaboração que me incentivaram sempre a continuar em frente.

- Ao Centro Nacional de Pesquisa (CNPq) pela ajuda financeira através da bolsa de estudos segundo o processo 132710/2000-5.

- Ao Dr. Marcelo B.P. Amato, pela constante colaboração.

- À professora Dra. Joyce Bevilacqua, e ao professor Dr. Marcos de S. Guerra Tsuzuki, pelas sugestões para melhorar o conteúdo deste trabalho.

- Às dependências, professores e funcionários do Departamento de Engenharia Mecânica da Escola Politécnica da Universidade de São Paulo que facilitaram a realização deste trabalho, especialmente aos professores Dr. Agenor T. Fleury e ao professor Dr. Linilson R. Padovese pela sua colaboração.

- À Claudia Patricia, que me acompanhou sempre, e me deu seu sorriso.

- Aos meus colegas de pós graduação que fizeram muito agradável minha estadia durante o tempo de desenvolvimento deste trabalho e dos quais aprendi sempre.

- À Iara muito obrigado pela ajuda, você é minha amiga.

- Aos meus amigos Antonio, Elvys, Julio, Gustavo, Mario, Modesto e Silmara, pelos valiosos aportes da vida.

- A todos aqueles que estiveram ao meu lado e me ajudaram para a boa execução deste trabalho. 


\section{SUMÁRIO}

Página

LISTA DE FIGURAS $\ldots \ldots \ldots \ldots \ldots \ldots \ldots \ldots \ldots \ldots$ iv

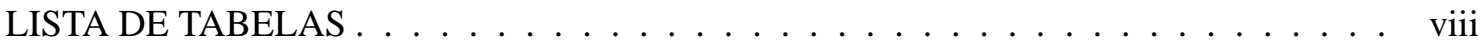

LISTA DE ABREVIATURAS E SÍMBOLOS . . . . . . . . . . . . . .

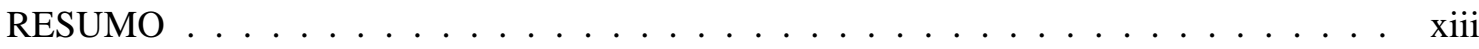

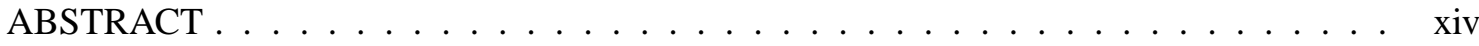

INTRODUÇÃO

1 OBJETIVO 3

2 REVISÃO BIBLIOGRÁFICA 4

2.1 Generalidades das Técnicas de Imagens Médicas . . . . . . . . . . . . . . . . . 4

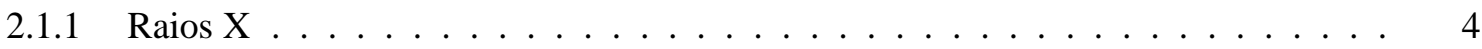

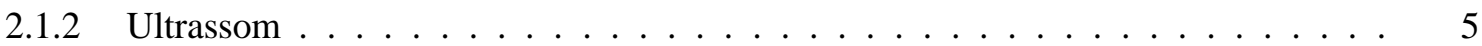

2.1 .3 Medicina Nuclear . . . . . . . . . . . . . . . . . . . 5

2.1.4 Tomografia Computadorizada $(\mathrm{CT}) \ldots \ldots \ldots \ldots$

2.1.5 Ressonância Magnética $(\mathrm{RM}) \quad \ldots \ldots \ldots$. . . . . . . . . . 7

2.1.6 Tomografia por Impedância Elétrica (TIE) . . . . . . . . . . . . . . . . . . 7

2.2 Estudos Experimentais da TIE . . . . . . . . . . . . . . . . 9

2.3 Métodos e Algoritmos . . . . . . . . . . . . . . . . . . . . . . 10

2.4 Filtro de Kalman . . . . . . . . . . . . . . . . . . . . . . . . . . 11

\section{BANCADA EXPERIMENTAL PARA TOMOGRAFIA}

POR IMPEDÂNCIA ELÉTRICA 13

3.1 Tomógrafo por Impedância Elétrica $\ldots \ldots \ldots$. . . . . . . . . . . . . . . . 13

3.1.1 Princípios de Operação do Mark I . . . . . . . . . . . . . . . . . . . . . . . . 13

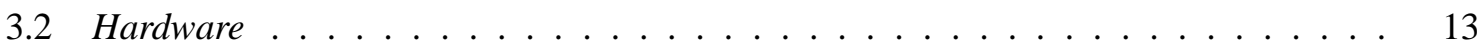

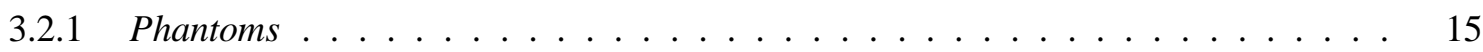

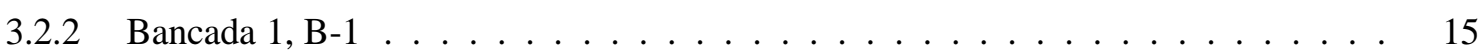

3.2 .3 Bancada $2, \mathrm{~B}-2 \ldots \ldots \ldots \ldots \ldots \ldots$

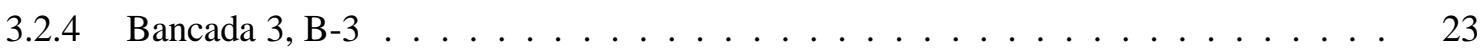


4.1 Metodologia Para a Solução do Problema Direto . . . . . . . . . . . . . . . . . . 27

4.1 .1 Discretização e Seleção do Tipo de Elemento . . . . . . . . . . . . . . . . . 27

4.1.2 Cálculo da Matriz de Condutividade Local . . . . . . . . . . . . . . . 28

4.1.3 Matriz de Condutividade Global . . . . . . . . . . . . . . . . 33

4.1 .4 Cálculo das Voltagens . . . . . . . . . . . . . . . . . 34

4.2 Metodologia Para a Solução do Problema Inverso . . . . . . . . . . . . . . . 35

4.2.1 Cálculo da Matriz de Condutividade Local . . . . . . . . . . . . . . 36

4.2.2 Matriz de Condutividade Global Simbólica . . . . . . . . . . . . . . . . 38

4.3 Metodologia Para a Solução do Problema Inverso,

Aplicando o Filtro de Kalman . . . . . . . . . . . . . . . . . . . 38

$4.3 .1 \quad$ Matriz de Sensibilidade $H \ldots \ldots \ldots \ldots \ldots$

4.3.2 Equações do Filtro de Kalman . . . . . . . . . . . . . . . . . . . . . 40

4.3 .3 Condição Inicial do Vetor $\hat{\sigma}_{o}^{(+)} \ldots \ldots \ldots \ldots \ldots \ldots \ldots$

4.3 .4 Condição Inicial da Matriz $P_{o}^{(+)} \ldots \ldots \ldots \ldots \ldots \ldots \ldots$

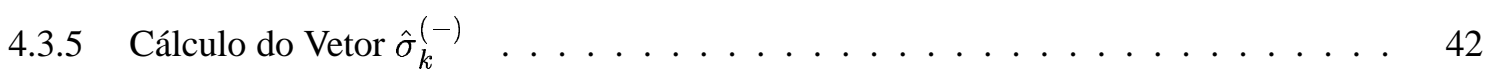

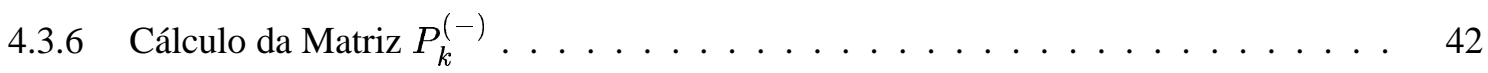

4.3 .7 Cálculo do Ganho de Kalman $G_{k} \ldots \ldots \ldots \ldots \ldots$

4.3 .8 Cálculo da Matriz $P_{k}^{(+)} \ldots \ldots \ldots \ldots \ldots \ldots \ldots \ldots$

4.3 .9 Cálculo do Vetor $\hat{\sigma}_{k}^{(+)} \ldots \ldots \ldots \ldots \ldots \ldots \ldots \ldots \ldots$

4.3 .10 Critério de Convergência . . . . . . . . . . . . . . 46

4.3 .11 Simulações . . . . . . . . . . . . . . . . . . . . 46

4.3.12 Simulações com novos parâmetros $P_{o}^{(+)}, Q$ e $R \ldots \ldots \ldots$. . . . . . . 51

\section{RENUMERAÇÃO DA MALHA E}

MATRIZ DE CONDUTIVIDADE DIAGONAL DE BANDA

5.1 Renumeração da Malha . . . . . . . . . . . . . . . . . . . . . 59

5.2 Matriz de Condutividade Diagonal de Banda . . . . . . . . . . . . . . . . 60

5.3 Cinco Casos de Estudo de Malhas Renumeradas . . . . . . . . . . . . . . . . . 62

6 REFINAMENTO ITERATIVO

6.1 Aplicação do Refinamento Iterativo . . . . . . . . . . . . . . . . . . 75 
7 RESULTADOS E DISCUSSÃO

7.1 Estimativa das Condutividades nos 32 Eletrodos . . . . . . . . . . . . . . . 80

7.2 Cinco Regiões de Interesse (5 ROIs) … . . . . . . . . . . . . . . . . . . 84

7.2.1 Estimativa de Impeditividade em 5 ROIs, Alg_Esparsa $\ldots \ldots$. . . . . . . . . 86

7.2.2 Estimativa de Impeditividade em 5 ROIs, Alg_RRI _ . . . . . . . . . . . . . . 86

7.3 Oito Regiões de Interesse $(8$ ROIs $) \ldots \ldots \ldots \ldots \ldots \ldots$

7.3.1 Estimativa de Impeditividade em 8 ROIs, Alg_Esparsa _ . . . . . . . . . . . . . . 89

7.3.2 Estimativa de Impeditividade em 8 ROIs, Alg_RRI . . . . . . . . . . . . . . . . . 89

7.4 Dezesseis Regiões de Interesse $(16$ ROIs $) \ldots \ldots \ldots$

7.4.1 Estimativa de Impeditividade em 16 ROIs, Alg_Esparsa . . . . . . . . . . . . . . . 94

7.4.2 $\quad$ Estimativa de Impeditividade em 16 ROIs, Alg_RRI . . . . . . . . . . . . . . . . . 94

7.5 Comparação dos Resultados para 5,8 e 16 ROIs . . . . . . . . . . . . . . . . . . . 96

7.6 Avaliação dos Resultados . . . . . . . . . . . . . . . . . . . . . . . . . . 98

8 CONCLUSÕES E SUGESTÕES PARA TRABALHOS FUTUROS 103

REFERÊNCIAS BIBLIOGRÁFICAS $\quad 105$

APÊNDICES I I

A FORMULAÇÃO DO FILTRO DE KALMAN I

B ALGORITMO PARA A APLICAÇÃO DO REFINAMENTO ITERATIVO VII

C ALGORITMO IMPLEMENTADO PARA TOMOGRAFIA POR IMPEDÂNCIA ELÉTRICA $\quad X$

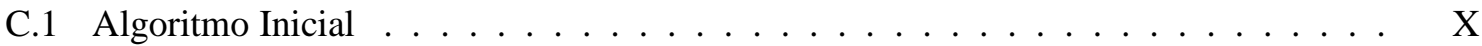

C.2 Algoritmo em Linguagem $\mathrm{C} \ldots \ldots \ldots \ldots \ldots \ldots$ XI

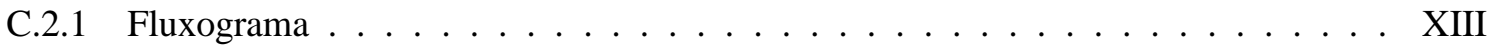

C.2.2 Algoritmo Principal Desenvolvido . . . . . . . . . . . . . . XVI 


\section{LISTA DE FIGURAS}

Página

2.1 Tomografia computadorizada do tórax $[9] \ldots \ldots \ldots \ldots \ldots$

3.1 Arranjo de eletrodos na tomografia por impedância elétrica. . . . . . . . . . . . . . . 14

3.2 Diagrama do princípio de operação do tomografo Mark I, Sheffield. . . . . . . . . . . 14

3.3 Circuito de injeção de corrente, amplificador referência : H65AB-LM12CLK. Com $\mathrm{R}=2.5 K \Omega$ e $\mathrm{P}=90 \Omega$, onde a corrente (I) é determinada pelo valor do potenciômetro P e a tensão de entrada. . . . . . . . . . . . . . . . . . . . . . 16

3.4 Esquema do circuito de amplificação da bancada B-1. Com $\mathrm{R}=100 K \Omega, \mathrm{R} 1=1 M \Omega$, $\mathrm{P}=10 K \Omega, \mathrm{C}=2.2 \mu F$ e $\mathrm{D}=\mathrm{BZX} 79 \mathrm{C} 16 \mathrm{PH} \ldots \ldots \ldots \ldots$

3.5 Montagem da primeira bancada B-1 de tomografia por impedância elétrica, com phan-

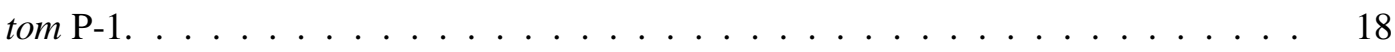

3.6 Sistema de excitação e sistema de medição da primeira bancada B-1 . . . . . . . . . 18

3.7 Filtro passa alta, utilizado na segunda bancada, B-2. Com $\mathrm{R}=1 M \Omega, \mathrm{C}=2.2 \mu F$. . . . 20

3.8 Circuito do multiplexador DG-506AKN, segundo especificações do fabricante Vishay Siliconix, com conector DB 25 . . . . . . . . . . . . . . . . 21

3.9 Montagem da segunda bancada B-2 de tomografia por impedância elétrica. . . . . . 22

3.10 Segunda bancada B-2 de tomografia por impedância elétrica com demodulação analógica, conversor de voltagem para corrente e multiplexadores. . . . . . . . . . . . . 22

3.11 Phantom P-3 com solução salina, de diâmetro $22 \mathrm{~cm}$, com 32 eletrodos e objeto cilíndrico de vidro de diâmetro $6.1 \mathrm{~cm}$, da terceira bancada B-3. . . . . . . . . . . 24

3.12 Montagem da terceira bancada B-3 de tomografia por impedância elétrica A. multímetro; B. fonte de alimentação; C. gerador de sinal e fonte de corrente; D. objeto cilíndrico de vidro; E. arranjo de eletrodos (32); F. recipiente com solução salina. . . . . . . . . 25

4.1 Malha de 4 elementos, 5 nós e 4 eletrodos. . . . . . . . . . . . . . . . . 28

4.2 Elemento triangular 1 da malha de elementos finitos. . . . . . . . . . . . . . 29

4.3 Elemento triangular 2 da malha de elementos finitos. . . . . . . . . . . . . 31

4.4 Elemento triangular 3 da malha de elementos finitos. . . . . . . . . . . . . 32

4.5 Elemento triangular 4 da malha de elementos finitos. . . . . . . . . . . 33

4.6 Cálculo das voltagens dos nós no problema direto. . . . . . . . . . . . . 35 
4.7 Simulação 1. Variação das condutividades dos 4 elementos, segundo os parâmetros da Tabela 4.1, sem adicionar ruído ao vetor $v_{k}^{(n f)}$ após 90 iterações. Distribuição de condutividade homogênea esperada de $0.1(\Omega m)^{-1} \ldots \ldots \ldots$. . . . . . . . . . 48

4.8 Resíduo normalizado, $r_{v}$, da simulação 1 . Verifica-se $-8.49 \leq r_{v} \leq 8.49 \ldots \ldots$. . 48

4.9 Simulação 2. Variação das condutividades dos 4 elementos segundo parâmetros da Tabela 4.2, após 90 iterações, adicionando ruído branco Gaussiano de média nula ao vetor $v_{k}^{(n f)}$ com desvio padrão $5 \%$ da máxima voltagem calculada por meio do problema direto. Distribuição de condutividade homogênea esperada de $0.1(\Omega m)^{-1}$. $\quad 50$

4.10 Resíduo normalizado, $r_{v}$, da simulação 2 . Verifica-se $-8.49 \leq r_{v} \leq 8.49 \ldots \ldots$. . 50

4.11 Simulação 3. Variação das condutividades dos 4 elementos segundo parâmetros da Tabela 4.3, após 90 iterações, adicionando ruído branco Gaussiano de média nula ao vetor $v_{k}^{(n f)}$ com desvio padrão $7 \%$ da máxima voltagem calculada por meio do problema direto. Distribuição de condutividade esperada, não homogênea. . . . . . . . 52

4.12 Resíduo normalizado, $r_{v}$, da simulação 3. Verifica-se $-6 \leq r_{v} \leq 6 . \ldots \ldots 2$

4.13 Simulação 1 com novos parâmetros. Variação das condutividades dos 4 elementos, segundo parâmetros da Tabela 4.4, sem adicionar ruído ao vetor $v_{k}^{(n f)}$, após 90 iterações. Distribuição de condutividade homogênea esperada de $0.1(\Omega m)^{-1}$. . . . . . . . . . 54

4.14 Simulação 2 com novos parâmetros. Variação das condutividades dos 4 elementos segundo parâmetros da Tabela 4.5. Após 90 iterações, adicionando ruído branco Gaussiano de média nula ao vetor $v_{k}^{(n f)}$ com desvio padrão $5 \%$ da máxima voltagem calculada por meio do problema direto. Distribuição de condutividade homogênea esperada de $0.1(\Omega m)^{-1}$. . . . . . . . . . . . . . . . . .

4.15 Simulação 3 com novos parâmetros. Variação das condutividades dos 4 elementos segundo parâmetros da Tabela 4.6. Após 90 iterações, adicionando ruído branco Gaussiano de média nula ao vetor $v_{k}^{(n f)}$ com desvio padrão $7 \%$ da máxima voltagem calculada por meio do problema direto. Distribuição de condutividade esperada, não

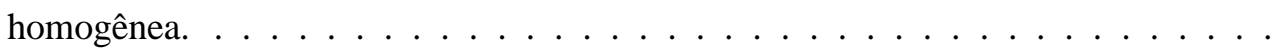

4.16 Comparação dos resultados das variações das condutividades dos 4 elementos. Parâmetros $P_{o}^{(+)}, Q$ e $R$ obtidos por experimentação numérica : (a) Simulação 1, (c) Simulação 2, (e) Simulação 3. E com novos parâmetros $P_{o}^{(+)}$e $Q$ obtidos a partir das considerações de Brogan : (b) Simulação 1, (d) Simulação 2, (f) Simulação 3. . . . . . . . . . . . . . . 
5.1 Malha de elementos finitos com 4 elementos e 5 nós, (a) malha inicial, (b) malha

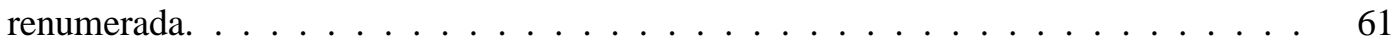

5.2 Caso 2. Malha inicial de elementos finitos com 32 elementos, 25 nós e 16 eletrodos. . 64

5.3 Caso 2. Malha renumerada de elementos finitos com 32 elementos, 25 nós e 16 eletrodos. 65

5.4 Caso 3. Malha inicial de elementos finitos com 96 elementos, 65 nós e 16 eletrodos. . 66

5.5 Caso 3. Malha renumerada de elementos finitos com 96 elementos, 65 nós e 16 eletrodos. 67

5.6 Caso 4. Malha inicial de elementos finitos com 118 elementos, 92 nós e 16 eletrodos. 68

5.7 Caso 4. Malha renumerada de elementos finitos com 118 elementos, 92 nós e 16

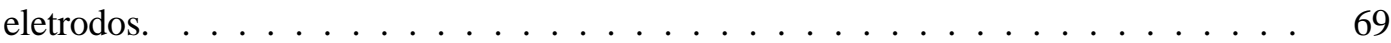

5.8 Caso 5. Malha inicial de elementos finitos com 182 elementos, 156 nós e 32 eletrodos. 70

5.9 Caso 5. Malha renumerada de elementos finitos com 182 elementos, 156 nós e 32 eletrodos. .............................. 71

5.10 Tamanho das colunas das matrizes $Y$ e $Y c$ para os cinco casos de estudo. . . . . . . 72

5.11 Tempo em segundos para o cálculo da inversa da matriz de condutividade. . . . . . . 73

7.1 Malha de elementos finitos do phantom com 182 elementos, 156 nós e 32 eletrodos. Phantom de diâmetro $22 \mathrm{~cm}$ com o objeto cilíndrico de vidro de diâmetro $6 \mathrm{~cm}$. . . . 78

7.2 Estimativas dos parâmetros no tempo nas 33 ROIs, onde 32 ROIs representam os 32 eletrodos. Para a malha com 182 elementos, 156 nós e 32 eletrodos. Gráfico de 3200 iterações. . . . . . . . . . . . . . . . . . . .

7.3 Agrupamento 1. 5 regiões de interesse para a malha de elementos finitos que representa o phantom em 182 elementos, 156 nós e 32 eletrodos. Na região número 2 encontra-se o objeto cilíndrico de vidro. . . . . . . . . . . . . .

7.4 Comparação dos resultados do Agrupamento 1, 5 ROIs. Estimativas de impeditividade no tempo para as 5 ROIs : (a) Alg_Esparsa, (c) Alg_RRI. Representação tridimensional do vetor de impeditividade das 5 ROIs na última iteração para : (b) Alg Esparsa. (d) Alg_RRI. Valores obtidos depois de 1920 iterações. Em todos os graficos a região com maior valor de impeditividade corresponde à região 2, na qual está o objeto. Malha de 182 elementos, 156 nós e 32 eletrodos. . . . . . . . . . . . . . . . . . . . . . . . 
7.5 Comparação dos resultados do Agrupamento 1, 5 ROIs. Estimativas de impeditividade no tempo para as 5 ROIs : (a) Alg_Esparsa, (c) Alg_RRI. Representação tridimensional do vetor de impeditividade das 5 ROIs na última iteração para : (b) Alg Esparsa. (d) Alg_RRI. Valores obtidos depois de 12800 iterações. Em todos os graficos, a região com maior valor de impeditividade corresponde à região 2, na qual está o objeto. Malha de 182 elementos, 156 nós e 32 eletrodos. . . . . . . . . . . . . . . .

7.6 Agrupamento 2. 8 regiões de interesse para a malha de 182 elementos, 156 nós e 32 eletrodos. Entre as regiões número 2, 3 e 4 encontra-se o objeto de vidro. . . . . . . . 90

7.7 Comparação dos resultados do Agrupamento 2, 8 ROIs. Estimativas de impeditividade no tempo para as 8 ROIs : (a) Alg_Esparsa, (c) Alg_RRI. Representação tridimensional do vetor de impeditividade das 8 ROIs na última iteração para : (b) Alg Esparsa. (d) Alg_RRI. Valores obtidos depois de 1920 iterações. Em todos os graficos as regiões com maior impeditividade correspondem às regiões 2,3 e 4 onde se encontra o objeto. Malha de 182 elementos, 156 nós e 32 eletrodos. . . . . . . . . . . . . . . . . . . .

7.8 Agrupamento 3. 16 regiões de interesse para a malha de 182 elementos, 156 nós e 32 eletrodos. Entre as regiões número 5, 9 e 12 encontra-se o objeto de vidro. . . . . . . .

7.9 Comparação dos resultados do Agrupamento 3, 16 ROIs. Estimativas de impeditividade no tempo para as 16 ROIs : (a) Alg_Esparsa, (c) Alg_RRI. Representação tridimensional do vetor de impeditividade das 16 ROIs na última iteração para : (b) Alg_Esparsa. (d) Alg_RRI. Valores obtidos depois de 1920 iterações. Em todos os gráficos as regiões com maior impeditividade correspondem às regiões 5, 9 e 12 onde se encontra o objeto. Malha de 182 elementos, 156 nós e 32 eletrodos. . . . . . . . . .

7.10 Comparação dos resultados da representação tridimensional do vetor de impeditividade para Alg_Esparsa de : (a) 5 ROIs, (c) 8 ROIs, (e) 16 ROIs; e Alg_RRI de : (b) 5 ROIs, (d) 8 ROIs, (f) 16 ROIs. Malha com 182 elementos, 156 nós e 32 eletrodos. . .

7.11 Comparação dos resultados do resíduo normalizado para Alg Esparsa de (a) 5 ROIs, (c) 8 ROIs, (e) 16 ROIs e Alg_RRI de (b) 5 ROIs, (d) 8 ROIs, (f) 16 ROIs. Malha com 182 elementos, 156 nós e 32 eletrodos. Consideração de convergência verificada para as duas versões.

A.1 Esquema do Filtro de Kalman $[8] . \ldots \ldots \ldots \ldots \ldots \ldots$ VI

C.1 Fluxograma, parte $1 . \ldots \ldots \ldots \ldots \ldots \ldots \ldots \ldots$ XIV

C.2 Continuação do Fluxograma. . . . . . . . . . . . . . . . . . . . . XV 


\section{LISTA DE TABELAS}

Página

4.1 Simulação 1. Parâmetros para o Filtro de Kalman aplicado ao exemplo da Figura 4.1 , sem adicionar ruído ao vetor $v_{k}^{(n f)}$. Distribuição de condutividades homogênea

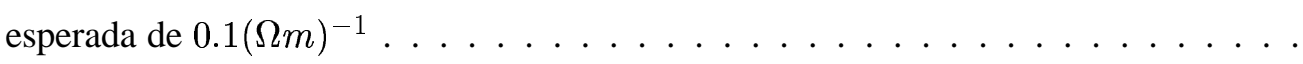

4.2 Simulação 2. Parâmetros para o Filtro de Kalman aplicado ao exemplo da Figura 4.1, adicionando ruído branco Gaussiano de média nula ao vetor $v_{k}^{(n f)}$ com desvio padrão $5 \%$ da máxima voltagem calculada por meio do problema direto. Distribuição de condutividades homogênea esperada de $0.1(\Omega m)^{-1} \ldots \ldots$. . . . . . . . .

4.3 Simulação 3. Parâmetros para o Filtro de Kalman aplicado ao exemplo da Figura 4.1, adicionando ruído branco Gaussiano de média nula ao vetor $v_{k}^{(n f)}$ com desvio padrão $7 \%$ da máxima voltagem calculada por meio do problema direto. Distribuição de condutividades esperada, não homogênea $\ldots \ldots \ldots \ldots \ldots$

4.4 Simulação 1. Novos parâmetros do Filtro de Kalman aplicado ao exemplo da Figura 4.1 , sem adicionar ruído ao vetor $v_{k}^{(n f)}$. Distribuição de condutividades homogênea esperada de $0.1(\Omega m)^{-1} \ldots \ldots \ldots \ldots \ldots \ldots \ldots \ldots \ldots \ldots \ldots$

4.5 Simulação 2. Novos parâmetros do Filtro de Kalman aplicado ao exemplo da Figura 4.1, adicionando ruído branco Gaussiano de média nula ao vetor $v_{k}^{(n f)}$ com desvio padrão $5 \%$ da máxima voltagem calculada por meio do problema direto. Distribuição de condutividade homogênea esperada de $0.1(\Omega m)^{-1} \ldots \ldots$. . . . . . . . . . . . 54

4.6 Simulação 3. Novos parâmetros do Filtro de Kalman aplicado ao exemplo da Figura 4.1, adicionando ruído branco Gaussiano de média nula ao vetor $v_{k}^{(n f)}$ com desvio padrão $7 \%$ da máxima voltagem calculada por meio do problema direto. Distribuição de condutividade esperada, não homogênea . . . . . . . . . . . . . . 56

5.1 Dimensões das matrizes de condutividade, nas formas esparsa e compacta para os cinco casos de estudo . . . . . . . . . . . . . . . . . . . . 63

5.2 Tempo em segundos para o cálculo da inversa da matriz de condutividade . . . . . 72

7.1 Resumo dos três agrupamentos estudados e parâmetros do Filtro Estendido de Kalman para 5, 8 ou 16 ROIs na malha de elementos finitos com 182 elementos, 156 nós e 32

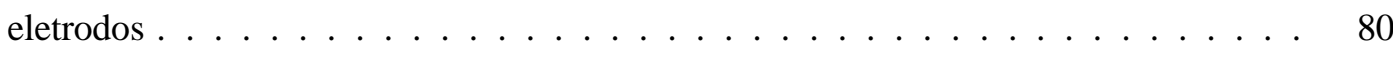

7.2 Parâmetros utilizados para obter uma estimativa da condutividade nos 32 eletrodos . 81 
7.3 Estimativas de parâmetros nos 32 eletrodos, obtidas com a malha agrupada em 33 ROIs a partir de dados experimentais . . . . . . . . . . . . . . . . 82

7.4 Elementos que constituem as 5 regiões de interesse para a malha de elementos finitos da Figura 7.3 e parâmetros para o Filtro de Kalman . . . . . . . . . . . . . . . . . . 84

7.5 Elementos que constituem as 8 ROIs . . . . . . . . . . . . . . . . . . . . . . 89

7.6 Elementos que constituem as 16 regiões de interesse para a malha de elementos finitos da Figura 7.8 e parâmetros para o Filtro de Kalman . . . . . . . . . . . . . . . . . . 92

7.7 Resultados de impeditividade nas ROIs obtidos pelas duas versões. Alg_Esparsa e Alg_RRI . . . . . . . . . . . . . . . . . . . . . . . 97

7.8 Limite superior na perturbação na inversa da matriz de condutividade . . . . . . . 102 


\title{
LISTA DE ABREVIATURAS E SÍMBOLOS
}

\author{
A - área de cada elemento triangular \\ Alg_Esparsa - algoritmo inicial, com matriz de condutividade esparsa \\ Alg_RRI - algoritmo com malha renumerada e refinamento iterativo \\ ATP - applied potencial tomography \\ $B$ \\ - matriz da geometria de cada elemento, com função de interpolação linear \\ B-1 - primeira bancada de tomografia por impedância elétrica \\ B-2 - segunda bancada de tomografia por impedância elétrica \\ B-3 - terceira bancada de tomografia por impedância elétrica \\ CT - tomografia computadorizada \\ D - matriz com as propriedades do material, condutividade homogênea para os \\ elementos \\ I - matriz identidade \\ ne - número de elementos planos triangulares \\ nf - número de eletrodos igualmente distribuídos \\ nm - número de escalares contidos na matriz de condutividade local, \\ dos elementos triangulares \\ nme - número de escalares contidos na matriz de condutividade local \\ dos elementos retangulares (eletrodos) \\ nn - total de nós na malha \\ $p \quad$ - número de padrões de corrente \\ P-1 - primeiro phantom \\ P-2 - segundo phantom \\ P-3 - terceiro phantom \\ RM - ressonância magnética \\ ROIS - regiões de interesse \\ $t \quad$ - espessura \\ TIE - tomografia por impedância elétrica \\ tpc - tipo de padrão de corrente \\ UTI - unidade de terapia intensiva \\ $Y \quad$ - matriz de condutividade global \\ $Y^{-1} \quad$ - inversa da matriz de condutividade global \\ $Y b \quad$ - matriz de condutividade diagonal de banda \\ Yc - matriz de condutividade diagonal de banda na forma compacta
}




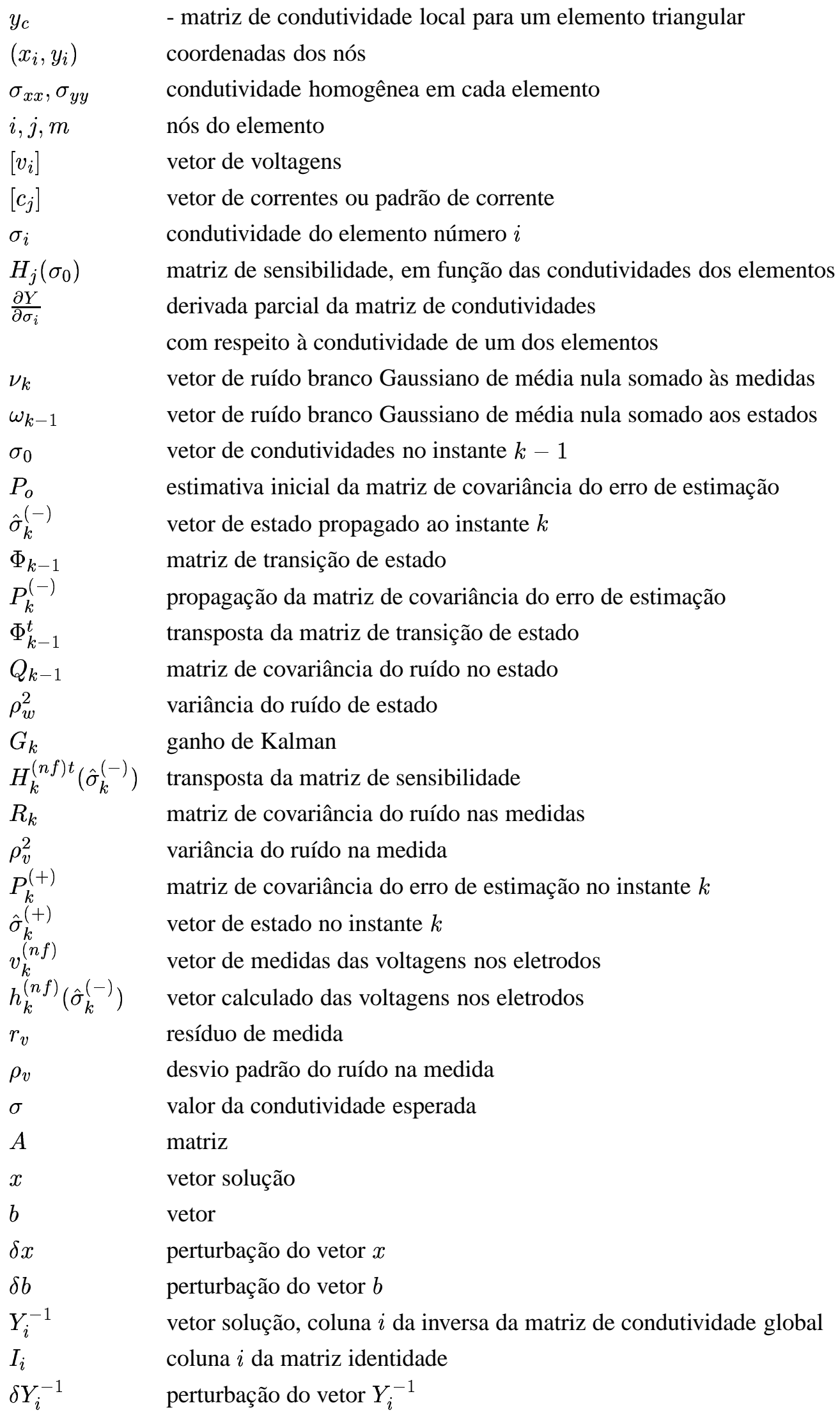


$\delta I_{i} \quad$ perturbação do vetor $I_{i}$

$\hat{\rho}_{(o)}^{(+)} \quad$ vetor de impedâncias para os elementos (ou ROIS) no instante $k-1$

$\hat{\rho}^{(+)} \quad$ estimativa do vetor de impedâncias para as regiões de interesse

$\delta A \quad$ perturbação da matriz $A$

$\|A\| \quad$ norma de $A$

$\|\delta A\| \quad$ norma de $\delta A$

$k(A) \quad$ número de condição da matriz $A$

$\delta Y^{-1} \quad$ perturbação da inversa da matriz de condutividade $Y$

$\left\|Y^{-1}\right\| \quad$ norma de $Y^{-1}$

$\left\|\delta Y^{-1}\right\| \quad$ norma de $\delta Y^{-1}$

$k\left(Y^{-1}\right) \quad$ número de condição de $Y^{-1}$

$\delta H \quad$ perturbação da matriz de sensibilidade

$\|H\| \quad$ norma da matriz $H$

$\|\delta H\| \quad$ norma de $\delta H$

$x_{k\left(n_{x} 1\right)} \quad$ vetor processo de estado no tempo $t_{k}$

$\Phi_{k\left(n_{x} n\right)} \quad$ matriz relacionando $x_{k}$ a $x_{k+1}$ na ausência de função forçante.

Se $x_{k}$ for uma amostra do processo contínuo, $\Phi_{k}$ será a matriz de

transição do estado

$w_{k\left(n_{x} 1\right)} \quad$ ruído branco, com estrutura de covariância conhecida

$z_{k\left(m_{x} 1\right)} \quad$ vetor da medida no tempo $t_{k}$

$H_{k\left(m_{x} n\right)} \quad$ matriz de sensibilidade no instante $k$

$v_{k\left(m_{x} 1\right)} \quad$ erro de medida, sequiência de ruído branco

com estrutura de covariância conhecida e não correlacionada com a seqüência $w_{k}$

$e_{k}^{(-)} \quad$ erro de estimação

$P_{k}^{(-)} \quad$ matriz de covariância do erro de estimação propagada ao instante $k$

$\hat{x}_{k}^{(-)} \quad$ estimativa inicial do vetor de estado

$K_{k} \quad$ ganho de Kalman

$\hat{x}_{k+1}^{(-)} \quad$ estado propagado ao instante $k+1$

$P_{k+1}^{(-)} \quad$ matriz de covariância de estado propagado ao instante $k+1$

$P_{k} \quad$ matriz de covariância no instante $k$

$\hat{x}_{k} \quad$ vetor de estado no instante $k$ 


\section{REDUÇÃO DE ERRO NUMÉRICO NO FILTRO ESTENDIDO DE KALMAN APLICADO À TOMOGRAFIA POR IMPEDÂNCIA ELÉTRICA}

autor: NELSON ANTONIO VANEGAS MOLINA orientador: Prof. Dr. RAUL GONZALEZ LIMA

\section{RESUMO}

A Tomografia por Impedância Elétrica (TIE) aplica-se no monitoramento contínuo e detecção de alterações pulmonares sérias. Principalmente no ambiente das unidades de terapia intensiva (UTI) para a avaliação das condições do paciente em estado crítico submetido à ventilação artificial sem que seja necessário retirar o paciente da UTI e dos diferentes instrumentos de assistência à vida. A técnica permite estimar alterações de impedância nos pulmões.

O objetivo deste trabalho é diminuir o erro numérico num algoritmo desenvolvido para TIE, utilizando o Filtro Estendido de Kalman. Especificamente, esse algoritmo aplica-se na a obtenção de imagens dos pulmões do corpo humano. Para realizar tal objetivo foram projetados phantoms compostos por um recipiente circular com solução salina, dentro do qual é colado um objeto cilíndrico de vidro e 32 eletrodos localizados no contorno do recipiente.

Foi desenvolvido um algoritmo em linguagem C, utilizando a técnica de Filtro Estendido de Kalman para estimação de parâmetros de um modelo de elementos finitos. Foram implementados o procedimento de renumeração da malha de elementos finitos, com o objetivo de obter uma matriz de condutividade de banda, e o procedimento de melhoramento iterativo da solução para diminuir o erro numérico de soluções de sistemas lineares.

Foram comparados dois algoritmos, um utilizando matriz de condutividade esparsa Alg Esparsa e outro com matriz de condutividade de banda limitada, obtida por renumeração da malha, e aplicando refinamento iterativo na solução de sistemas lineares, Alg_RRI. Obtiveram-se melhores estimativas de impedância e uma melhor estabilidade do algoritmo do Filtro de Kalman com o algoritmo Alg_RRI. O erro numérico na inversa da matriz de condutividade e o erro numérico na matriz de sensibilidade são significativamente menores quando se utiliza renumeração da malha e refinamento iterativo da solução de sistemas lineares. A redução de erro numérico nestas matrizes leva a melhores imagens. 


\section{NUMERICAL ERROR REDUCTION IN THE EXTENDED KALMAN FILTER APPLIED TO ELECTRICAL IMPEDANCE TOMOGRAPHY}

Author: NELSON ANTONIO VANEGAS MOLINA

Adviser: Prof. Dr. RAUL GONZALEZ LIMA

\section{ABSTRACT}

The Electrical Impedance Tomography (EIT) is applied for the continuing monitoring and detection of serious pulmonar change. It may be used in intensive care units for the evaluation of patient condition in critical state submitted to artificial ventilation. It is not necessary to leave the intensive care unit and disconnect life assist devices. This technique allow estimation of impedance distribution on a cross section of the thorax.

The main of this work is the reduction of numerical error in the Kalman Filter for EIT image estimation. Specifically, this algorithm may be applied for estimating lunge impedance distribution. To obtain this objective a phantom was developed. It is constituted by a cilindrical container with saline solution, a glass object is glued to the container, and 32 electrodes attached to the container wall.

An algorithm in C language, using the Extended Kalman Filter technique was developed, it is a parameter estimation procedure. Mesh renumbering, to obtain a band limited conductivity matrix and the iterative improvement of the solution of linear systems were implemented.

The estimation of impedance distribution was performed. Two different algorithms were considered. One algorithm uses a sparse conductivity matrix, Alg_sparse. Another algorithm uses a band limited conductivity matrix and iterative refinement of the solution of linear systems, Alg_RRI. Better impedance estimation and better stability of Kalman Filter algorithm was obtained using Alg_RRI. The numerical error on the inverse of the conductivity matrix and the numerical error on the sensitivity matrix were smaller on algorithm Alg_RRI. The numerical error reduction on the conductivity matrix and on the sensitivity matrix produced better images. 


\section{INTRODUÇÃO}

A tomografia por impedância elétrica é um método particular de obter imagens por meio da injeção de corrente elétrica de baixa potência no objeto a caracterizar. A imagem é obtida a partir dos potenciais elétricos ou correntes detectados na superfície do objeto. Na década de 80 a Universidade de Sheffield [7] desenvolveu o que hoje se conhece como Tomografia por Impedância Elétrica (TIE), a qual fornece imagens relativas.

Com esta técnica pretende-se representar a distribuição de condutividade da seção do corpo humano em cujo contorno são obtidas as medidas dos potenciais elétricos. Sendo este problema inverso não linear complica-se a sua solução. Para obter sua solução podem-se utilizar algoritmos iterativos, resolvendo em cada iteração o denominado problema direto. Este tipo de tomografia requer um conhecimento inicial da forma e do tamanho do objeto sob an álise, o tórax no presente estudo.

Destacam-se os termos de tomografia "absoluta" e "relativa". As denominadas tomografias "absolutas" permitem obter uma estimativa da impedância no interior do corpo humano. Na tomografia "relativa" estima-se a variação de condutividade entre dois instantes.

Se o parâmetro em função do qual se estuda a distribuição de condutividade é o tempo, obtêmse as denominadas imagens "dinâmicas". As imagens dinâmicas permitem estudar a evolução da impedância do coração durante o ciclo cardíaco, dos pulmões durante o ciclo respiratório ou dos pulmões durante o ciclo cardíaco.

Entre as aplicações médicas da TIE tem-se, o diagnóstico do pulmão e detecção de tumores. Seu estudo é importante, já que significa um novo procedimento de diagnóstico, para a detecção de alterações pulmonares sérias, no ambiente de unidades de terapia intensiva (UTI). Permite avaliar as condições do paciente em estado crítico submetido à ventilação artificial sem que seja necessário retirar o paciente da UTI ou desconectar equipamentos de assistência à vida.

Existe uma variedade de aplicações não clínicas da TIE. Estas incluem imagens de fluxo de fluidos multifase, a localização de depósitos minerais na terra, a distribuição de contaminantes na terra, a avaliação de ensaios não destrutivos de partes de máquinas e o controle de processos 
industriais tais como cura entre outros.

Já foram produzidos inúmeros trabalhos sobre TIE e, na atualidade, diferentes grupos de pesquisa do mundo vêm desenvolvendo a técnica. Entre eles destacam-se [3] :

- na Inglaterra, a Universidade de Sheffield desenvolveu um equipamento de tomografia por impedância elétrica baseado no algoritmo de backprojection;

- em Madison, a Universidade de Wisconsin desenvolveu um método de reconstrução de imagem utilizando o método de Newtom-Raphson;

- nos EUA encontra-se o grupo do Rensselear Polytechnic Institute que propôs um algoritmo rápido de reconstrução de imagens baseado no método de Newtom-Raphson, porém com apenas uma iteração, chamado FNOSER;

- na Finlândia, a Universidade de Kuopio desenvolveu um algoritmo baseado no Filtro de Kalman para estimar a distribuição de condutividades na TIE;

- e finalmente no Brasil, no Departamento de Engenharia Mecânica da EPUSP, vem sendo desenvolvido um algoritmo utilizando o Filtro Estendido de Kalman [32], entre outros. Este trabalho conta com a cooperação de dois grupos de pesquisa, um do Departamento de Pneumologia HCFMUSP liderado pelo Dr. Marcelo B. P. Amato, e outro do Departamento de Matemática Aplicada IME da Universidade de São Paulo, liderado pela professora Dra. Joyce Bevilacqua, conforme projeto temático Fapesp [3] Novas Estratégias em Ventilação Artificial: Diagnóstico e Prevenção do Barotrauma/Biotrauma através da Tomografia de Impedância Elétrica.

O propósito deste trabalho é a redução de erro numérico num algoritmo desenvolvido para tomografia por impedância elétrica, utilizando o Filtro Estendido de Kalman.

Para atingir os objetivos foram realizadas as seguintes tarefas :

- revisão bibliografica da TIE;

- construção de três bancadas experimentais, compostas por phantom, condicionadores de sinais, sistema de injeção de corrente e sistema de aquisição de potenciais nos eletrodos;

- desenvolvimento de algoritmos no software Octave, baseados no Filtro Estendido de Kalman;

- desenvolvimento de algoritmos em linguagem $\mathrm{C}$, implementando nele melhoras para atingir o objetivo da redução de erro numérico;

- desenvolvimento de algoritmos em linguagem C, agrupando os elementos da malha em 5, 8 ou 16 regiões de interesse. 


\section{Capítulo 1}

\section{OBJETIVO}

O objetivo deste trabalho é a redução de erro numérico num algoritmo desenvolvido para tomografia por impedância elétrica, utilizando o Filtro Estendido de Kalman.

A metodologia básica consiste em :

- projeto e desenvolvimento de bancada experimental para tomografia por impedância elétrica por meio da qual adquiriram-se dados experimentais para fornecer ao algoritmo. Procura-se identificar um objeto dentro do phantom e estimar a distribuição de impedância;

- implementação e utilização do algoritmo do Filtro Estendido de Kalman aplicado à TIE, Alg_Esparsa;

- implementação e utilização de um algoritmo do Filtro Estendido de Kalman aplicado à TIE com renumeração de malha e refinamento iterativo da solução de sistemas lineares, Alg_RRI;

- comparação dos resultados obtidos com os dois algoritmos utilizando 5, 8 ou 16 regiões de interesse. 


\section{Capítulo 2}

\section{REVISÃO BIBLIOGRÁFICA}

\subsection{Generalidades das Técnicas de Imagens Médicas}

O diagnóstico por imagem utiliza imagens das estruturas internas do corpo humano para diagnóstico médico. As suas técnicas incluem o uso de raios $\mathrm{X}$ que emite radiação e radio nucleotídios, e também radiação não ionizante como ultrassom e ressonância magnética.

Embora o tema deste trabalho seja a Tomografia por Impedância Elétrica, a seguir descrevemse as principais técnicas de diagnóstico por imagem, o que permite estabelecer as diferenças existentes entre cada uma destas e finalmente ressaltar a aplicação da TIE.

\subsubsection{Raios X}

O físico Wilhelm Conrand Röntgen, reitor da universidade Wurzburg na Alemanha, na tarde de 8 de novembro de 1895, pesquisava o tubo de raios catódicos inventado pelo inglês William Crookes e por um acaso ao ligar o tubo encontrava-se perto uma placa de um material fluorescente chamado platino cianeto de bário a qual brilhou. O brilho persistiu mesmo quando se colocou um livro e uma folha de alumínio entre o tubo e a placa. No dia 22 de dezembro, fez a radiação atravessar por 15 minutos a mão de sua esposa, atingindo, do outro lado, uma placa fotográfica. Revelada a chapa, viam-se nela as sombras dos ossos, essa foi a primeira radiografia da história [33, 34].

Os raios X são ondas eletromagnéticas de comprimento de onda muito curto, da ordem de $10^{-9} \mathrm{~m}$.

Atualmente, com modernos aparelhos de raios X, imagens são feitas em milésimos de segundos e a dose de raios $\mathrm{X}$ usada é menor. Também, as técnicas de raios $\mathrm{X}$ têm maior resolução espacial e detalhes de contraste. Isto melhorou a qualidade da imagem permitindo diagnósticos de 
maior número de patologias [12].

É usado principalmente para obter imagens de ossos em diagnóstico de fraturas.

\subsubsection{Ultrassom}

O início das técnicas do ultrassom vieram com a descoberta do efeito piezoelétrico de certos cristais, por Pierre Curie e Jacques Curie na França em 1880 [1].

Ultrassom ou Ultrassonografia em medicina, é o uso de ondas sonoras de alta frequiência para visualizar estruturas internas do corpo humano. O processo do ultrassom envolve um transdutor que é colocado no paciente na área de interesse, por exemplo o fígado, o transdutor transmite e recebe o som. As ondas de som produzidas penetram no interior do corpo e batem no orgão fazendo com que elas voltem e sejam detectadas pelo transdutor. Tecidos distintos refletem estas ondas diferentemente, causando um sinal típico o qual é medido e transformado em imagem.

O método permite o exame de órgãos abdominais, pélvicos e estruturas superficiais como mama, tireóide, globo ocular e sistema músculo esquelético [10].

\subsubsection{Medicina Nuclear}

A medicina nuclear está baseada no descobrimento dos raios gama, por Enrico Fermi (1935). Os primeiros estudos de medicina nuclear foram feitos em 1950 usando aparelhos especiais chamados "câmaras gama". A medicina nuclear requer a introdução oral ou intravenosa de químicos com substâncias de baixíssimo nível de radiação (chamados rádio nucleotídios, radiofarmacêuticos ou radiotraçadores) no interior do corpo. A câmara gama tem um grande detector de cristal (chamado cristal de cintilação). Estes cristais detectam o sinal da radiação emitida e o converte em luz, a qual é convertida em um sinal eletrônico utilizado para formar uma imagem no computador [9].

A medicina nuclear é uma área especializada da radiologia que usa pequenas quantidades de agentes radioativos para examinar a estrutura e a função de vários orgãos, como o coração. Permite a visualização de orgãos e regiões não vistos com os raios X convencionais. Lesões que ocupam espaço, especialmente tumores e vários tipos de câncer, são melhor visualizados nas imagens de medicina nuclear. Também é importante no diagnóstico e tratamento de doençãs cardíacas.

\subsubsection{Tomografia Computadorizada (CT)}

O inglês Godfrey Hounsfield e o americano Allan Cormack durante os anos 70, criaram a tomografia computadorizada (CT). Este é um procedimento de diagnóstico por imagem que combina 
o uso de raios $\mathrm{X}$ com técnicas de reconstrução de imagens para produzir cortes tomográficos em planos horizontais ou verticais do corpo [9].

$\mathrm{Na}$ CT o paciente fica no interior de um grande anel que gira em torno dele. O anel emite e capta radiação de muitos ângulos diferentes. O resultado equivale a cerca de 130000 radiografias.

Mostra com detalhes qualquer parte do corpo humano, incluindo ossos, músculos, gordura e órgãos. Este método mede a atenuação dos raios X entrando no corpo humano desde diferentes ângulos. Destas medidas um algoritmo forma imagens de cortes tomográficos. Esta técnica elimina o problema que existe no raio X convencional onde as sombras se superpõem. Além disso, permite que tecidos como o fígado e o rim possam ser claramente diferenciados um do outro quando reconstruídos pelo computador [10].

A CT é a principal máquina na maioria dos departamentos radiológicos e centros de diagnósticos. Desde sua invenção observam-se grandes avanços de tecnologia e desempenho clínico.

Na Figura 2.1 mostra-se uma tomografia computadorizada do tórax, onde podem ser observados os pulmões esquerdo e direito, o coração, ossos e gordura, que são regiões de interesse neste estudo.

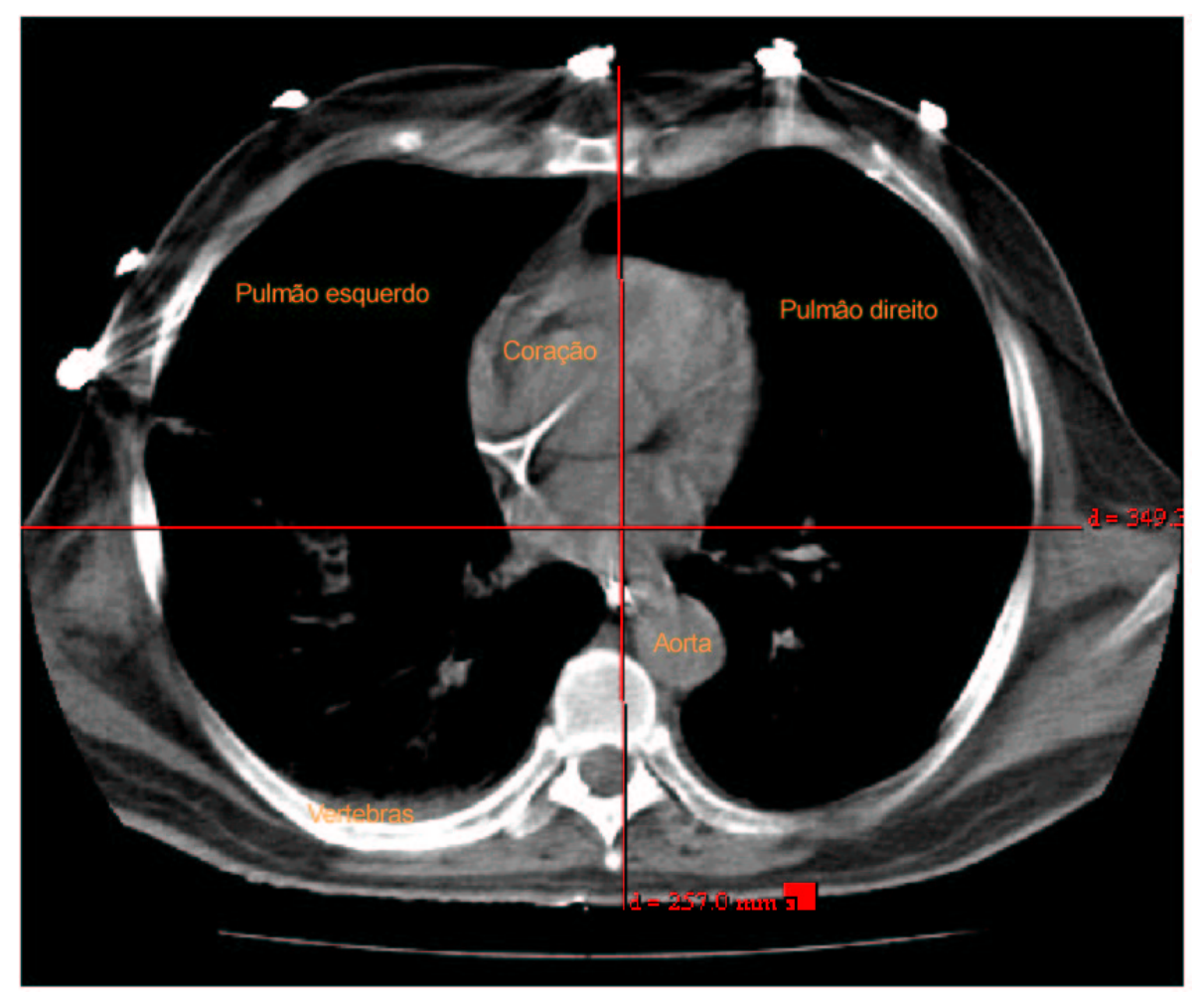

Figura 2.1: Tomografia computadorizada do tórax [9]. 


\subsubsection{Ressonância Magnética (RM)}

A ressonância magnética nuclear foi inicialmente estudada no início de 1970 e os primeiros protótipos foram testados clinicamente em pacientes em 1980. Foi inicialmente comercializado em 1984 e seu uso rapidamente se espalhou pelo mundo [9].

Na ressonância magnética nuclear, também chamada apenas de RM, o paciente é submetido a ondas de rádio sob um poderoso campo magnético. $\mathrm{RM}$ não usa raios $\mathrm{X}$ ou qualquer outro tipo de raio ionizante. Em lugar disso, é uma técnica que combina um campo magnético com freqüências de rádio. Átomos diferentes do corpo humano absorvem diferentes freqüências de ondas de rádio sob a influência de um campo magnético. A intensidade em que estes raios são absorvidos é medida e usada pelo computador para formar imagens internas do corpo humano.

Um dos benefícios da RM é que podem ser formadas imagens do corpo humano em quase todo tipo de orientação. São obtidas imagens bi-dimensionais que permitem fazer hologramas através de computação gráfica. Por exemplo, estes podem ser de grande auxílio para encontrar o local exato nas artérias coronárias que será submetido a angioplastia.

A RM tem sido utilizada no diagnóstico de cardiopatias, doenças vasculares, derrames cerebrais, no tratamento de lesões esportivas e diagnóstico de câncer de mama [11].

\subsubsection{Tomografia por Impedância Elétrica (TIE)}

Os antecedentes desta técnica podem ser encontrados nos trabalhos de Webster e Henderson [38] que tentaram reproduzir as técnicas de tomografia de raios $\mathrm{X}$ aplicando sinais elétricos de baixa frequiência. Foi no início da década de 80, que a Universidade de Sheffield [7] desenvolveu as bases do que hoje se entende como TIE.

As razões de interesse clínico da TIE são expostas pelo pesquisador Barber em Quantification in Impedance Imaging [5]. Ele descreve alguns exemplos de aplicações clínicas como estudos gástricos, funções pulmonares, funções cardíacas e mostra numa tabela a resistividade elétrica de vários tecidos, assim como futuros desenvolvimentos com esta técnica não invasiva.

Como sinais elétricos de baixa potência são usados como estíimulo, este método não causa nenhum tipo de aquecimento danoso aos tecidos e nem usa radiações ionizantes, sendo potencialmente útil para monitorar pacientes à beira do leito e na pediatria. A TIE tem algumas características muito atraentes na monitoração dos fluidos do pulmão, segundo Amato [2].

Na síndrome de angústia respiratória de adultos, a ventilação mecânica é usada para manter uma oxigenação adequada, mas pode causar danos ao pulmão. Diferentes procedimentos de ventilação tem sido desenvolvidas para reduzir o risco de trauma nos alvéolos durante a ventilação 
mecânica. O incremento da densidade do pulmão indica um aumento de alvéolos colapsados devido a edema pulmonar. Conhecer qual região do pulmão está colapsada ajuda no ajuste da ventilação mecânica e indica a severidade do edema pulmonar. A TIE registrando as variações da impedância do pulmão.

A condutividade e a permitividade elétrica interna permite determinar a localização de gás e sangue no interior do corpo humano. Estas propriedades elétricas são muito diferentes para o ar, os tecidos e o sangue; além disso, elas variam com frequências diferentes. Assim, um mapa da variação temporal das propriedades elétricas mostra regiões do pulmão que são ventiladas mas não irrigadas pelo sangue,por exemplo.

A seguir, apresenta-se um resumo das principais técnicas para obtenção de imagens médicas, descritas anteriormente.

Raios X [12, 33, 34] :

- descobertos em 1895 por Wilhelm Conrand Röntgen;

- imagens dos tecidos internos: ossos e órgãos;

- os raios X são ondas eletromagnéticas de comprimento de onda muito curto.

Ultrassom $[1,10]$ :

- efeito descoberto por Pierre e Jacques Curie, 1880;

- imagens de tecidos, orgãos abdominais, estruturas superficiais, sistema músculo esquelético;

- uso de ondas sonoras de alta frequiência para visualizar estruturas internas do corpo humano.

\section{Medicina Nuclear [9] :}

- inícios 1935 por Enrico Fermi, em 1950 primeiros estudos

em medicina nuclear;

- visualizar órgãos e regiões não visíveis através de raio X convencional. Tratameto de doençãs cardíacas, tumores e vários tipos de câncer;

- área especializada da radiologia que usa pequenas quantidades de agentes radioativos para visualizar a estrutura e a função de vários orgãos.

Tomografia Computadorizada (CT) $[9,10]$ :

- 1970, Godfrey Hounsfield e Allan Cormack;

- imagens de gordura, ossos e vasos sanguíneos;

- combina o uso de raios X com computação para produzir cortes tomográficos, em planos horizontais ou verticais do corpo.

Ressonância Magnética (RM) [9, 11] :

- início em 1970, protótipos 1980, comercializado 1984;

- imagens do corpo humano em quase todo tipo de orientação; 
- o paciente está sendo submetido a ondas de rádio sob um poderoso campo magnético, não usa raios $\mathrm{X}$ ou qualquer outro tipo de raio ionizante.

Tomografia por Impedância Elétrica (TIE) [2, 5, 7] :

- início 1978 Webster e Henderson, 1980 Universidade de Sheffield;

- aplicações : visualização do fluxo do sangue no tórax, na cabeça, ou no braço e ventilação pulmonar;

- obtenção de imagens da distribuição de impedância no interior do corpo.

Em um aparelho de raios $\mathrm{X}$ ou tomografia computadorizada, a radiação sai do tubo e passa através do corpo do paciente antes de ser detectado ou gravado num filme radiográfico ou computador. Nos aparelhos de medicina nuclear o material radioativo é introduzido no interior do paciente e logo detectado pela câmara gama. A radiação emitida do corpo são raios gama. Estes raios são semelhantes aos raios X mas têm um comprimento de onda menor. Os níveis de radiação envolvidos na medicina nuclear usualmente são mais baixos que os recebidos de um raios $\mathrm{X}$ convencional ou tomografia computadorizada.

Entre as técnicas, a CT é a única que tem habilidade de mostrar imagens de gordura, ossos e vasos sanguíneos. Por exemplo, o raios X convencional da cabeça mostrará apenas a calota craniana. Enquanto que a ressonância magnética é sensível a tecidos menos densos e vasos sanguíneos mas não fornece detalhes das estruturas ósseas.

A técnica RM, que usa ondas de rádio, é muito mais segura do que as técnicas que usam raios $\mathrm{X}$ ou raios gama, mas é mais cara.

Imagens médicas obtidas através de RM ou CT são usadas na avaliação do pulmão, mas é impossível sua utilização na UTI. E o deslocamento do paciente até equipamento de diagnóstico representa um risco muito grande para sua vida. Com o método de TIE é possível obter informação sobre a função do pulmão no leito de UTI.

\subsection{Estudos Experimentais da TIE}

Robert Guardo et al. (1991) em An Experimental Study in Electrical Impedance Tomography Using Backprojection Reconstrution [17], reportam um projeto experimental onde avaliam a importância das equipotenciais na técnica de backprojection, sendo esta a técnica que é utilizada nos atuais equipamentos e expõem os fundamentos matemáticos da técnica e a forma de implementar esta na TIE. O experimento é feito num recipiente com solução salina com dimensões similares ao tórax humano. O sistema do software é descrito. Consiste em três programas os quais são de protocolo, calibração e aquisição dos dados para finalmente calcular os valores de impedância. 
Robert W. M. Smith et al. (1995) em A Real- Time Electrical Impedance Tomography System for Clinical Use-Design and Preliminary Results [30], descrevem uma instrumentação que produz imagens da distribuição de impedâncias elétricas no corpo a uma taxa de 25 imagens por segundo, usado em ventilação do pulmão para observações em tempo real. O instrumento desenvolvido permite medições de impedância usando um arranjo de 16 eletrodos sobre a superfície do corpo e reconstrói imagens usando a técnica backprojection. O sistema de aquisição de dados e o processo de reconstrução de imagens é descrito. O sistema é baseado no trabalho desenvolvido por Brown e Barber [7]. A descrição é detalhada incluindo esquemas dos circuitos, especificações como o número de eletrodos, o número de canais, a fonte de corrente, a precisão das medições, o circuito de controle, o multiplexador da corrente, o procedimento de medição e da calibração do sistema.

Considerando a importância que têm os phantoms na tomografia por impedância elétrica, Luiz Felipe Fuks et al. (1991) em Detection and Imaging of Electric Conductivity and Permittivity at low Frequency [15], descrevem os resultados experimentais obtidos nos denominados phantoms. Estes objetos incluem tecidos vivos e materiais como cilindros de PVC, cilindros de alumínio e cilindros de cobre com óxido. Demonstra-se que o sistema tem a habilidade de distinguir os diferentes objetos e exibem-se as imagens da distribuição de resistividade.

Kevin Paulson et al. (1992) em A Hybrid Phantom for Electrical Impedance Tomography [27], argumentam que uma das considerações preliminares no estudo da tomografia é o projeto dos phantoms e que eles sejam modelos confiáveis e precisos. Descrevem um phantom com suas propriedades. O phantom para os diferentes testes de tomografia consiste de um tubo com diâmetro interior de $30 \mathrm{~cm}$ e $5 \mathrm{~cm}$ de altura. Igualmente espaçados ao seu redor, são instalados 32 lâminas em ouro-prata, que produzem uma impedância de contato uniforme e diminuem a corrosão.

\subsection{Métodos e Algoritmos}

David Isaacson et al. (1992) em Comments on Reconstruction Algorithms [21], descrevem vários algoritmos propostos para a estimação de condutividade elétrica no corpo através da medida de potencial elétrica na superfície do corpo e descreve e compara diversos algoritmos.

Normalmente a estimação de uma imagem de TIE depende de dois tipos de problemas segundo Yorkey et al. (1987), [39] : o problema direto e o inverso. A solução do problema direto permite o cálculo dos potenciais em qualquer ponto da superfície ou volume de estudo, dada uma aproximação da distribuição de resistividade e condições de contorno. O problema inverso trata de estimar a distribuição de resistividade através do conhecimento de medidas do potencial na fronteira, o que requer resolver iterativamente o problema direto, minimizando a diferença entre 
os potencias medidos e os calculados. A imagem de TIE é a estimativa da distribuição de resistividade ou de condutividade de uma secção transversal do volume estudado. Quando se trata do corpo humano, a seção transversal apresenta características não homogêneas, anisotrópicas e é composto por formas complexas e irregulares de órgãos e tecidos. Para a solução deste problema empregam-se duas famílias de algoritmos, entre outros : os baseados em backprojection e os baseados na matriz de sensibilidade [39]. O algoritmo backprojection consiste em construir imagens através de bandas homogêneas de corrente, estimadas por diferentes medidas ao longo da superfície externa do volume estudado. Já o algoritmo baseado na matriz de sensibilidade parte do princípio de que o produto dos valores de condutividade pela matriz de sensibilidade é equivalente a uma aproximação do problema direto.

Na pesquisa de Vauhkonen (1997-1998) [35, 36], estuda-se este problema através da formulação dinâmica para a TIE como um problema de estimação de estado, utilizando o filtro linearizado de Kalman e assim determinar a distribuição de impedância.

A dissertação de Trigo (2001) [32], propôs e desenvolveu um algoritmo para uma abordagem dinâmica alternativa utilizando o Filtro Estendido de Kalman para a estimação da distribuição de condutividades absolutas na TIE, tendo como base a abordagem dinâmica proposta por Vauhkonen. Além disso, por meio da formulação clássica, o problema de estimar variações de condutividades também foi abordado.

Neste trabalho foi desenvolvido um algoritmo empregando o Filtro Estendido de Kalman, implementando as técnicas de renumeração da malha de elementos finitos que discretiza o domínio, o refinamento iterativo para reduzir os erros numéricos para obter a distribuição de condutividades ou impedâncias num phantom a partir de dados experimentais.

\subsection{Filtro de Kalman}

Muitos trabalhos têm sido apresentados na literatura nos últimos anos utilizando a técnica de Filtro de Kalman, principalmente na engenharia de comunicação e navegação, e desempenha um papel importante na estimação da velocidade e destino de objetos que se movimentam com grande precisão.

Sorenson [31] escreve sobre o Filtro de Kalman :

"O Filtro de Kalman para alguns é somente uma técnica a ser utilizada no meio de um contexto global; para outros é uma resposta na procura de uma forma de resolver da melhor maneira seu problema específico; para outros ainda, representa um conjunto prático de procedimentos que podem ser usados para processar dados numéricos para obter estimativas de parâmetros e variáveis 
cujos valores são incertos".

Neste trabalho, o Filtro de Kalman é empregado para obter as estimativas da distribuição de condutividades ou de impedâncias ao formular o problema da TIE como um problema de estimação de estados. Em sua implementação, em primeiro lugar, o Filtro utiliza uma estimativa inicial dos estados e após a estimação de diversos coeficientes do modelo, esses coeficientes são utilizados de forma recursiva no modelo para estimar o estado presente do sistema, a solução é dada pelo vetor de estados que contém a distribuição de condutividades ou impedâncias.

O Filtro de Kalman é um algoritmo computacional recursivo ótimo [14] que tem como entrada medidas das voltagens disponíveis na TIE e como saídas as componentes do vetor de estado que representa a distribuição de condutividade. O Filtro é recursivo no sentido de que não há necessidade de se armazenar todas as informações recebidas, isto é, o algoritmo trabalha com as últimas estimativas de condutividades com as informações mais recentes. Sendo as novas medidas das voltagens que correspondem a um novo padrão de corrente.

Como razões para se utilizar o Filtro de Kalman, podem-se citar [26] :

- sua implementação exige pouco esforço computacional e pouca utilização de memória, já que o Filtro requer apenas uma álgebra matricial simples;

- o Filtro de Kalman fornece a estimativa de estado, como também o grau de confiança nesta estimativa;

- possui uma estrutura recursiva, o que permite o cálculo passo a passo dos estados estimados;

- embora seja utilizado em sistemas lineares pode ser adaptado para problemas não lineares, sendo então denominado Filtro de Kalman Estendido;

- devido ao rápido desenvolvimento dos computadores digitais, a técnica pode ser aplicada hoje em áreas onde não era econômica ou tecnicamente viáveis aplicá-la;

- pode-se aplicar o Filtro em problemas onde se tem uma modelagem sabidamente imperfeita ou simplificada.

Entretanto, algumas dificuldades podem aparecer na aplicação do método [26] :

- condicionamento numérico: o problema de TIE é numericamente mal condicionados (ou numericamente instáveis);

- sintonização : para o uso do método em toda sua potencialidade, alguns parâmetros devem ser ajustados, de acordo com cada aplicação particular.

Dadas as características do Filtro de Kalman, aplica-se esta técnica de estimação de parâmetros para obter as estimativas da distribuição de condutividade ou impedância. 


\section{Capítulo 3}

\section{BANCADA EXPERIMENTAL PARA TOMOGRAFIA POR IMPEDÂNCIA ELÉTRICA}

\subsection{Tomógrafo por Impedância Elétrica}

A configuração básica de um tomógrafo por impedância elétrica é constituída por eletrodos, sistema de aquisição de dados, sistema de injeção de corrente e os algoritmos para formar imagens de condutividade/resistividade.

\subsubsection{Princípios de Operação do Mark I}

O sistema de TIE desenvolvido pela Universidade de Sheffield, utiliza um arranjo de 16 eletrodos ao redor do tórax do paciente como se mostra na Figura 3.1. Uma corrente de $5 \mathrm{~mA} \mathrm{p} / \mathrm{p}$ com uma frequiência de $20 \mathrm{kHz}$ passa entre pares de eletrodos. A voltagem em cada eletrodo é medida, as medições são feitas seqüencialmente com um multiplexador. Uma vez realizadas as medições, a imagem é formada usando o backprojection.

Na Figura 3.2 observa-se um diagrama do princípio de operação da TIE que contém a configuração do tomógrafo Mark I.

\subsection{Hardware}

Nesta seção descreve-se a sucessão de phantoms, de sistemas de injeção e sistemas medida empregados. 


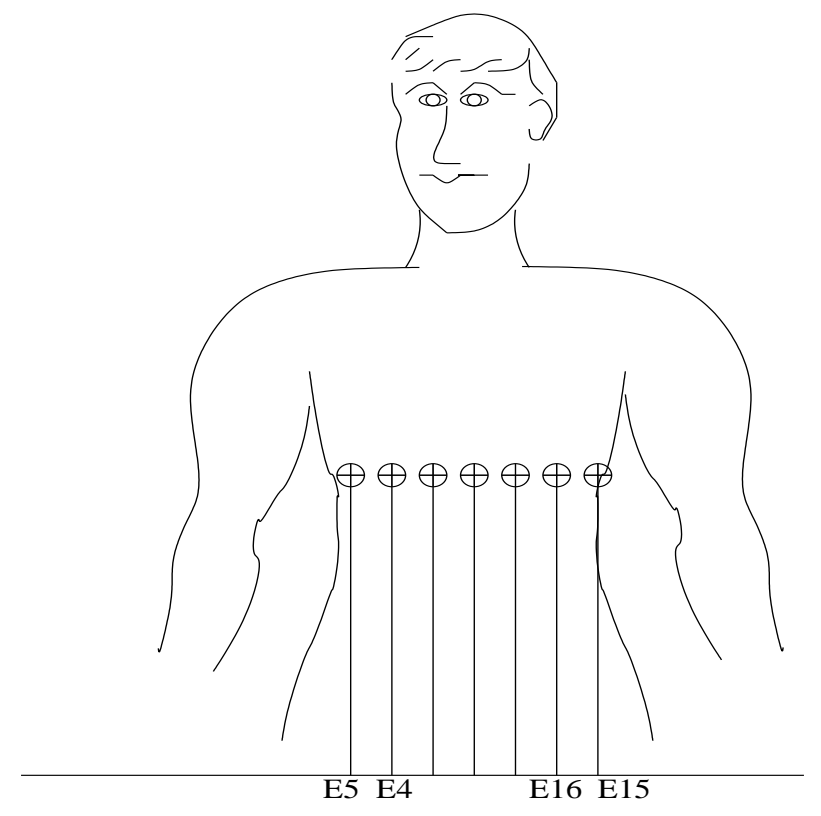

Figura 3.1: Arranjo de eletrodos na tomografia por impedância elétrica.

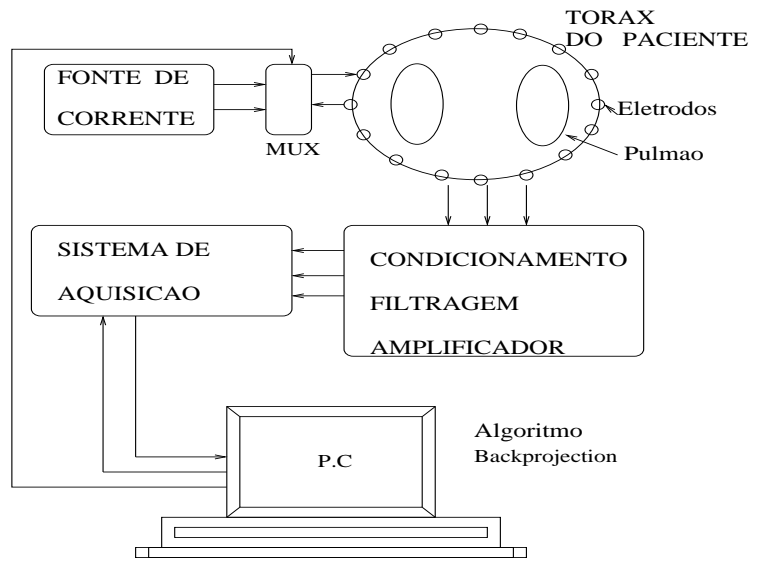

Figura 3.2: Diagrama do princípio de operação do tomografo Mark I, Sheffield. 
O sistema de completo, phantom, sistemas de injeção e sistemas medida será chamado de "bancada" neste trabalho.

\subsubsection{Phantoms}

O primeiro phantom, P-1, é formado por um recipiente circular de vidro, solução salina, cilindro de vidro imerso na solução salina e 16 eletrodos de bronze em forma cilindrica equi-espaçados no contorno interno do recipente de vidro.

O segundo phantom, P-2, é similar ao P-1, mas com 16 eletrodos de bronze em forma de lâminas.

O terceiro phantom, P-3, corrigiu uma série de problemas detectados nos phantoms $\mathrm{P}-1 \mathrm{e}$ P-2, os eletrodos foram espaçados com maior precisão, a largura dos eletrodos foi controlada com precisão $\perp_{-}^{+} 0.1 \mathrm{~mm}$ e utilizou-se um recipiente de parede lateral vertical. $\mathrm{O}$ phantom $\mathrm{P}-3$ foi construído a partir de um tubo de PVC de $22 \mathrm{~cm}$ de diâmetro interno sobre um círculo de vidro de $8 \mathrm{~mm}$ de espessura. Os 32 eletrodos foram posicionados através de parafusos.

O recipiente representa o tórax do paciente. Diferentes autores têm feito testes com recipientes circulares, para efeitos de simplificar o problema. Neste trabalho, para efeitos de comparação e avaliação, foram construídos recipientes de dimensões similares às estudadas [5, 15], mais isso não implica que não possam ser construídos outros tipos de recipientes com a geometria similar ao tórax.

\subsubsection{Bancada 1, B-1}

Para obter dados confiáveis precisou-se construir uma bancada de TIE na etapa inicial da pesquisa.

Nesta bancada os 16 eletrodos ficam dentro do recipiente. Nestes pontos metálicos injetaramse correntes com diferentes tipos de distribuições ou padrões e também foram feitas as medidas de voltagens necessárias pela aquisição de dados. Dentro do recipiente foi colado um objeto cilíndrico de dimensão menor e que representa o objeto cuja condutividade se quer estimar. $\mathrm{O}$ equivalente na TIE seria estimar a distribuição de condutividades nos pulmões e as variações de condutividade neles.

A bancada B-1 foi constituída pela seguinte instrumentação :

- 16 eletrodos cilíndricos com as seguintes dimensões : $1.5 \mathrm{~cm}$ de diâmetro e $3.0 \mathrm{~cm}$ de altura; construídos em bronze;

- recipiente de vidro (phantom) de dimensões : $26 \mathrm{~cm}$ de diâmetro na borda superior, $20 \mathrm{~cm}$ de diâmetro na base, $6 \mathrm{~cm}$ de altura; 
- solução salina : 1300 gramas de água destilada e 0.212 gramas de sal;

- objeto a estimar : cilíndrico, de vidro. Diâmetro $6.1 \mathrm{~cm}$ e $13.0 \mathrm{~cm}$ de altura;

- padrão de corrente aplicado : linearmente independente. Mantendo o mesmo eletrodo como referência (ou terra) e nos demais eletrodos aplicando-se a corrente em forma seqüencial. As voltagens medidas são as obtidas entre o eletrodo de referência e os demais eletrodos;

- Injeção de Corrente : No circuito abaixo (Figura 3.3), a tensão é convertida em corrente, mediante a utilização do amplificador operacional de potencia H65AB-LM12CLK como conversor de corrente. O circuito construído foi baseado em alguns exemplos de Horowitz [18].

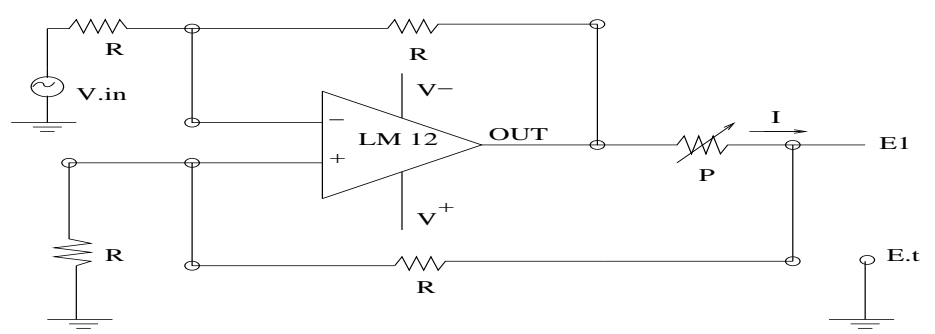

Figura 3.3: Circuito de injeção de corrente, amplificador referência : H65AB-LM12CLK. Com $\mathrm{R}=2.5 K \Omega$ e $\mathrm{P}=90 \Omega$, onde a corrente (I) é determinada pelo valor do potenciômetro $\mathrm{P}$ e a tensão de entrada.

- fonte de alimentação : marca Power Supply, modelo MPC-3003D;

- gerador do sinal : marca Dawer, modelo FG-200D;

- osciloscópio : marca Goldstar, modelo OS-9020A;

- circuito de amplificação : A estrutura inicial projetada é a que se mostra na Figura 3.4. Foi utilizado o amplificador operacional CA 3140. O $1^{\circ}$ amplificador operacional gera um sinal negativo constante para cancelar o off-set no sinal de voltagen do eletrodo. O $2^{\circ}$ amplificador operacional que está na configuração é somador inversor. O circuito conecta-se a um dos 16 canais de uma placa A/O com 10 bits de precisão. 


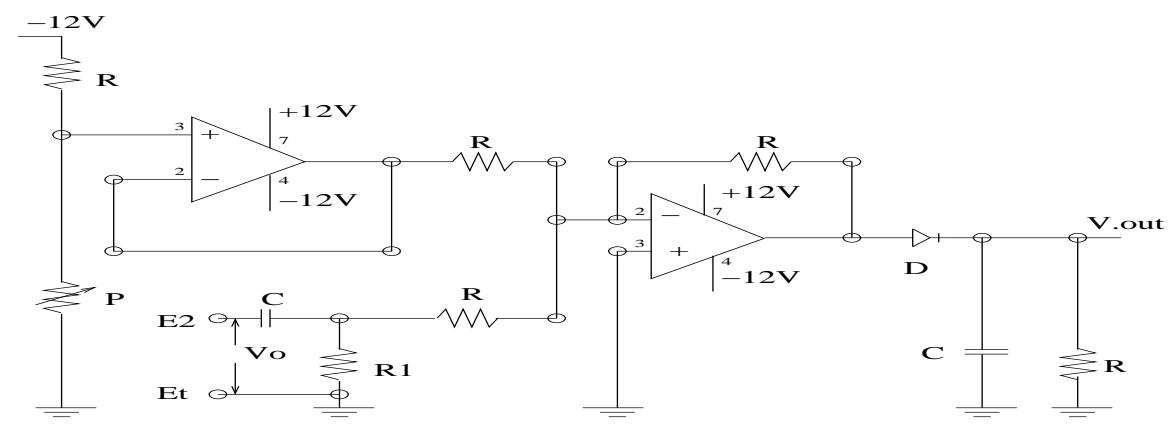

Figura 3.4: Esquema do circuito de amplificação da bancada B-1. Com $\mathrm{R}=100 K \Omega$, $\mathrm{R} 1=1 M \Omega, \mathrm{P}=10 K \Omega, \mathrm{C}=2.2 \mu F$ e $\mathrm{D}=\mathrm{BZX} 79 \mathrm{C} 16 \mathrm{PH}$.

- sistema multiplexador : foi montado o sistema com um multiplexador DG-506AKN, segundo especificações do fabricante Vishay Siliconix;

- sistema de aquisição : o sistema de aquisição se encarrega fundamentalmente de amostrar a tensão, sua conversão A/D e arquivamento do sinal digitalizado. Placa da marca Keithley MetraByte, modelo DAS- 1601, 16 canais;

- microcomputador.

Nas Figuras 3.5 e 3.6 mostra-se a primeira bancada, B-1, de TIE e o phantom P-1. 


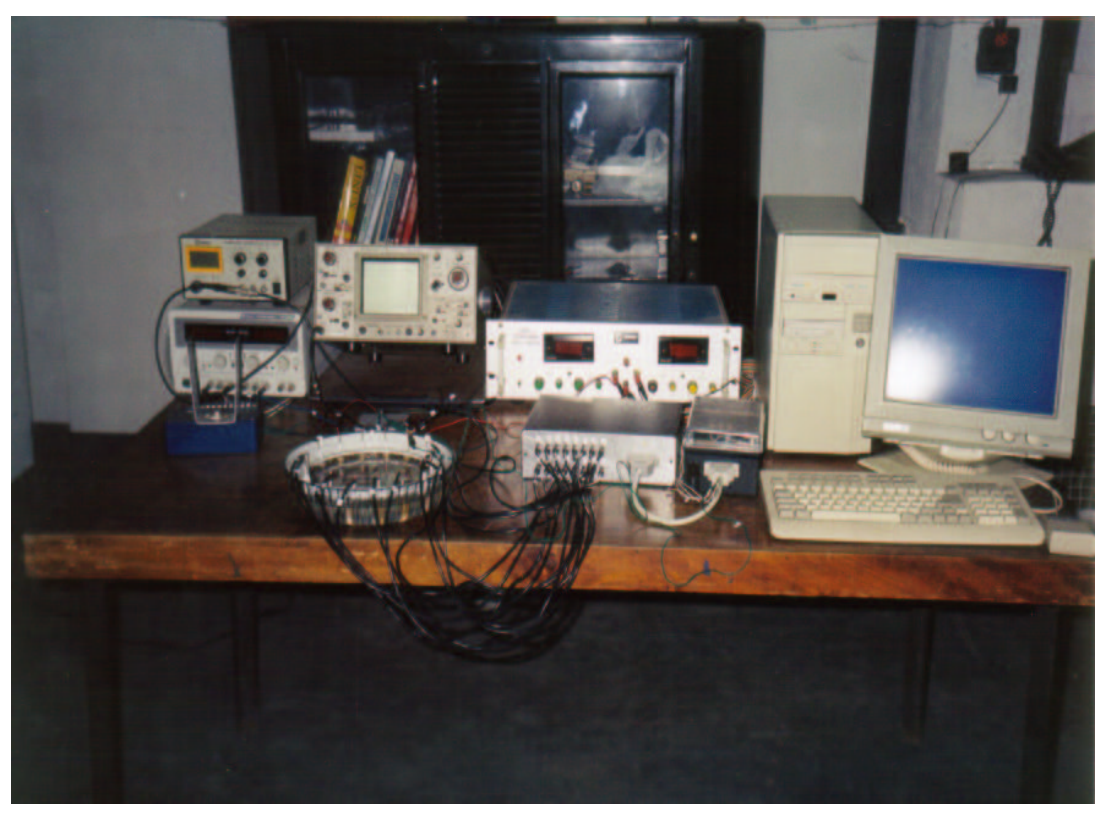

Figura 3.5: Montagem da primeira bancada B-1 de tomografia por impedância elétrica, com phantom $\mathrm{P}-1$.

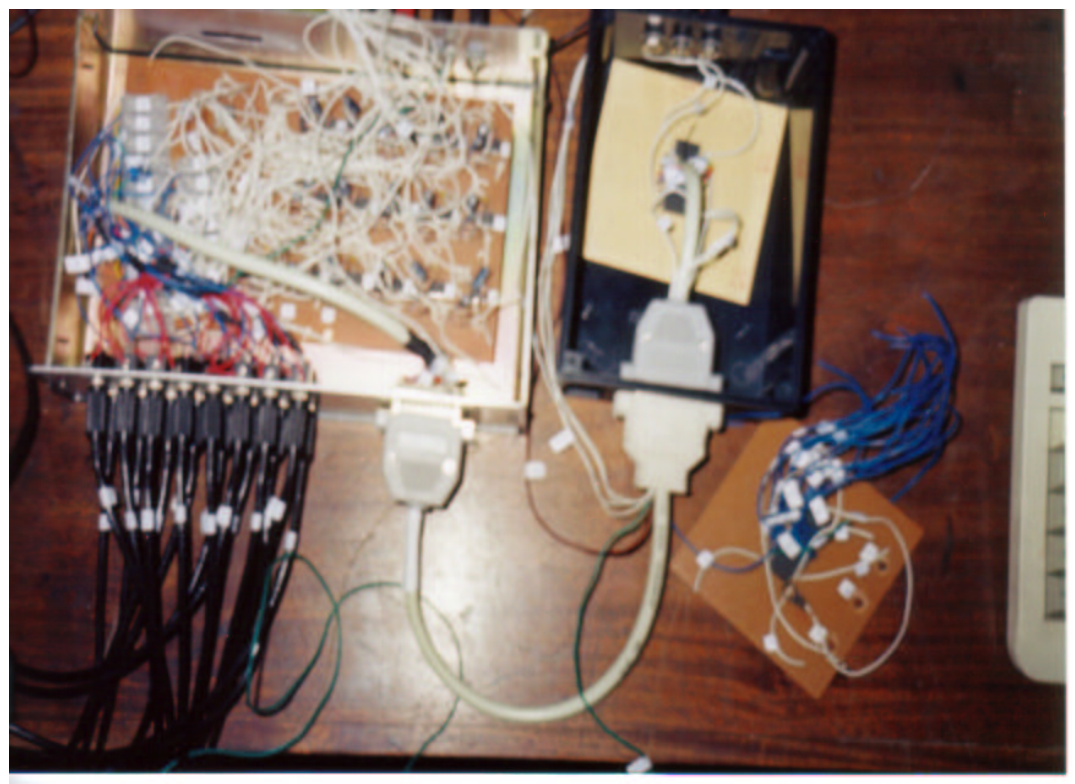

Figura 3.6: Sistema de excitação e sistema de medição da primeira bancada B-1. 


\subsubsection{Bancada 2, B-2}

Uma segunda bancada de TIE foi desenvolvido, usando um conjunto de 16 eletrodos compostos e dois multiplexadores, um multiplexador para definir qual eletrodo fica ligado ao terra e outro multiplexador para a injeção da corrente utilizando excitação diametral.

Nesta bancada foram adquiridas as medidas e as quais foram usadas na dissertação de Trigo [32].

O tipo dos eletrodos foi mudado neste phantom. Usaram-se chapas de bronze para minimizar erros identificados no primeiro phantom. Também foram feitos testes com um arranjo de 16 eletrodos no contorno do recipiente que representa o tórax. Além disso, foram mudadas as concentrações da solução para melhorar a condutividade e a resposta do sistema.

Esta bancada B-2 foi composta por :

- 16 eletrodos planos compostos de bronze, cada eletrodo é formado por um par de eletrodos: um eletrodo maior para a injeção de corrente e outro eletrodo pequeno para medir as voltagens, para minimizar a impedância de contato [19]. O eletrodo de injeção tem $6.5 \mathrm{~cm}$ de comprimento, $1.3 \mathrm{~cm}$ de largura e $0.1 \mathrm{~cm}$ de espessura, enquanto que o eletrodo para medida tem $6.5 \mathrm{~cm}$ de comprimento, $0.5 \mathrm{~cm}$ de largura e $0.1 \mathrm{~cm}$ de espessura;

- recipiente de vidro (phantom) : $26 \mathrm{~cm}$ de diâmetro na borda superior, $20 \mathrm{~cm}$ de diâmetro na base e $6 \mathrm{~cm}$ de altura;

- solução salina : 1300 gramas de água destilada com 0.300 gramas de $\mathrm{NaCl}$;

- dois objetos cilíndricos a estimar, ambos de vidro; o primeiro localizado próximo do contorno com diâmetro $6.1 \mathrm{~cm}$ e $13.0 \mathrm{~cm}$ de altura, e o segundo no centro, de diâmetro $4.7 \mathrm{~cm}$ e 10.0 $\mathrm{cm}$ de altura;

- padrão de corrente aplicado : diametral. No qual tem-se qualquer eletrodo como eletrodo de referência e aplica-se corrente no eletrodo diametralmente oposto e medem-se as voltagens entre o eletrodo de referência e cada um dos demais eletrodos, assim até ter todos os eletrodos como referência;

- Injeção de Corrente : o mesma configuração utilizada na primeira bancada, conforme a Figura 3.3;

- fonte de alimentação : marca Power Supply, modelo MPC-3003D;

- gerador do sinal : marca Dawer, modelo FG-200D;

- osciloscópio;

- sistema de Filtro : Filtro passa alta, utilizada na entrada de cada canal da placa de aquisição A/D, conforme a Figura 3.7, se tem 16 circuitos equivalentes que permitem obter as medidas de 
voltagens nos eletrodos.

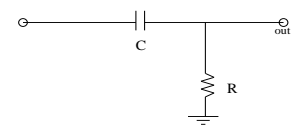

Figura 3.7: Filtro passa alta, utilizado na segunda bancada, $\mathrm{B}-2$. Com $\mathrm{R}=1 M \Omega, \mathrm{C}=2.2 \mu F$.

- sistema com dois multiplexadores : DG-506AKN, fabricante Vishay Siliconix, um para definir o eletrodo de referência (ou terra) e outro para definir o eletrodo de injeção de corrente, segundo mostra-se a Figura 3.8;

- interface de I/O : Keithley MetraByte, modelo DAS-1601[24], 16 canais single-ended;

- microcomputador.

As Figuras 3.9 e 3.10 mostram a segunda bancada B-2 de TIE. 


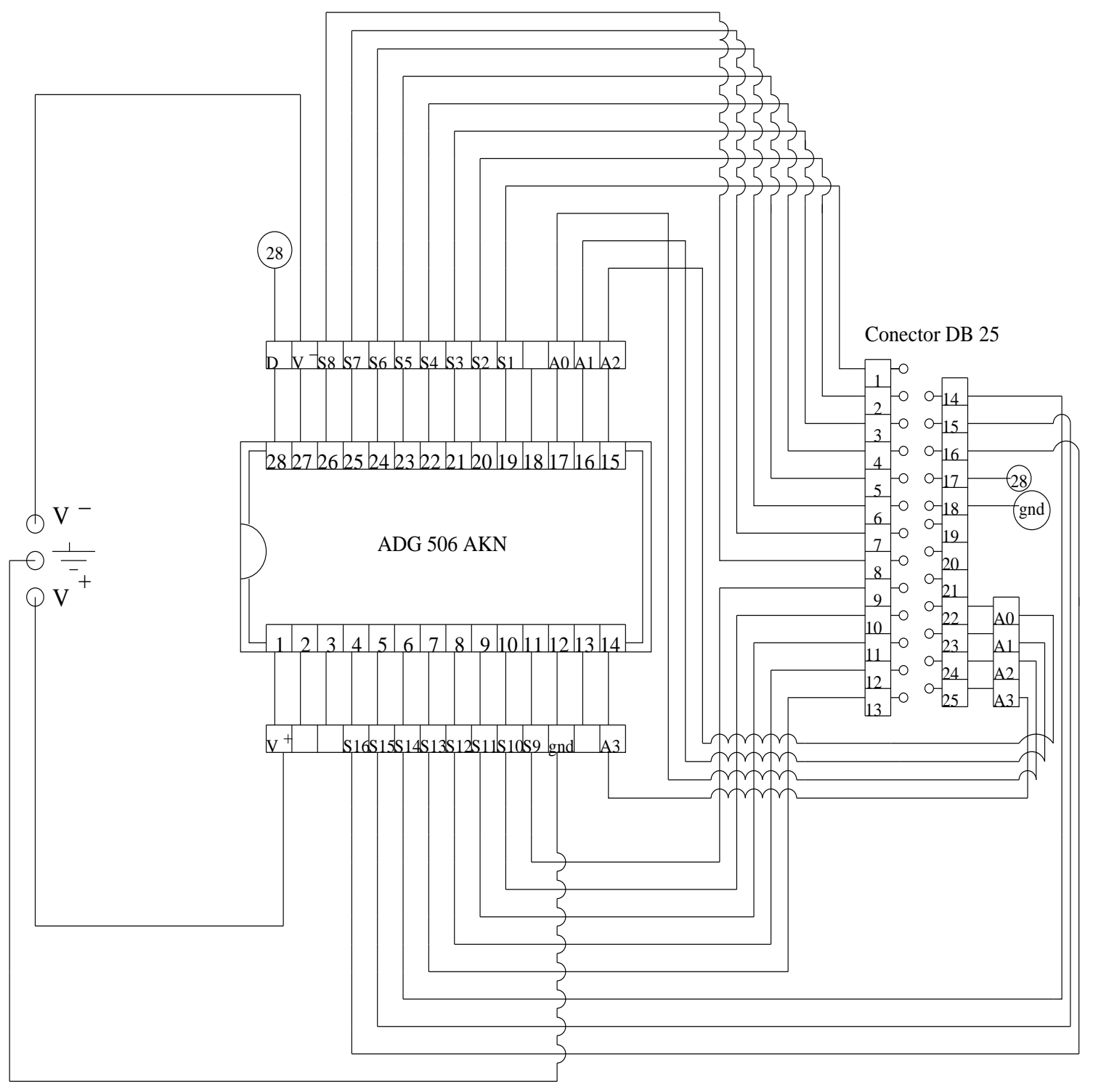

Figura 3.8: Circuito do multiplexador DG-506AKN, segundo especificações do fabricante Vishay Siliconix, com conector DB 25. 


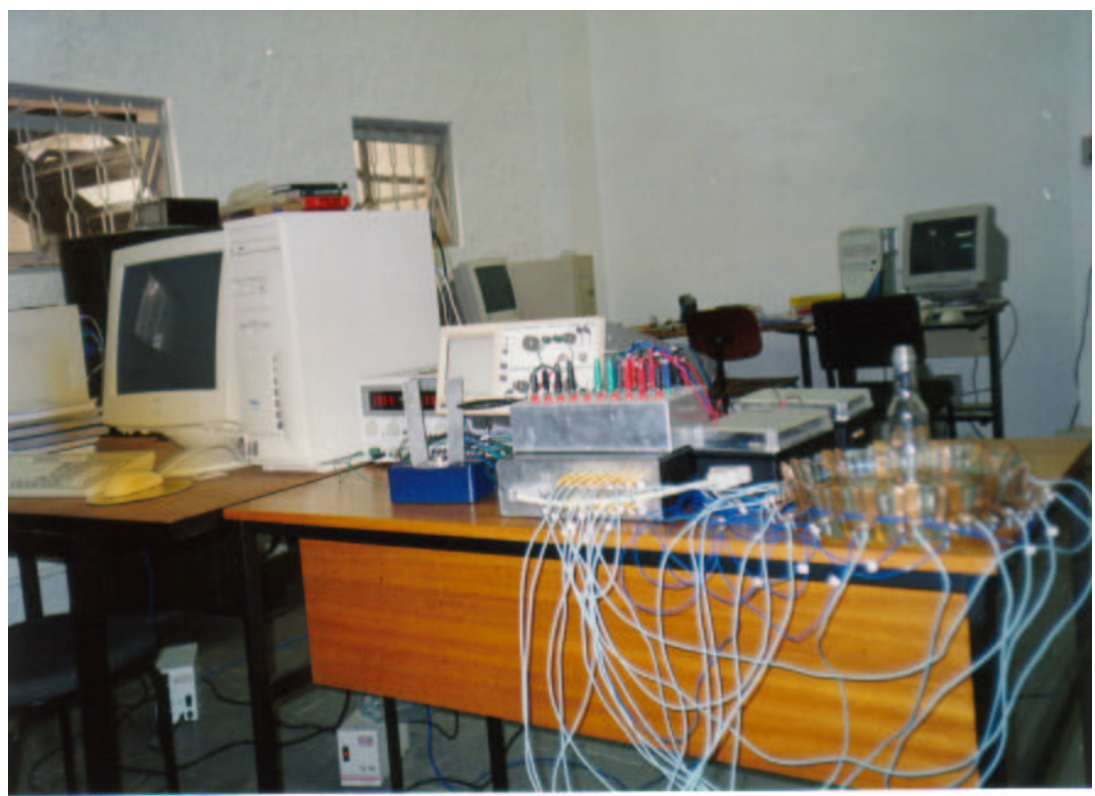

Figura 3.9: Montagem da segunda bancada B-2 de tomografia por impedância elétrica.

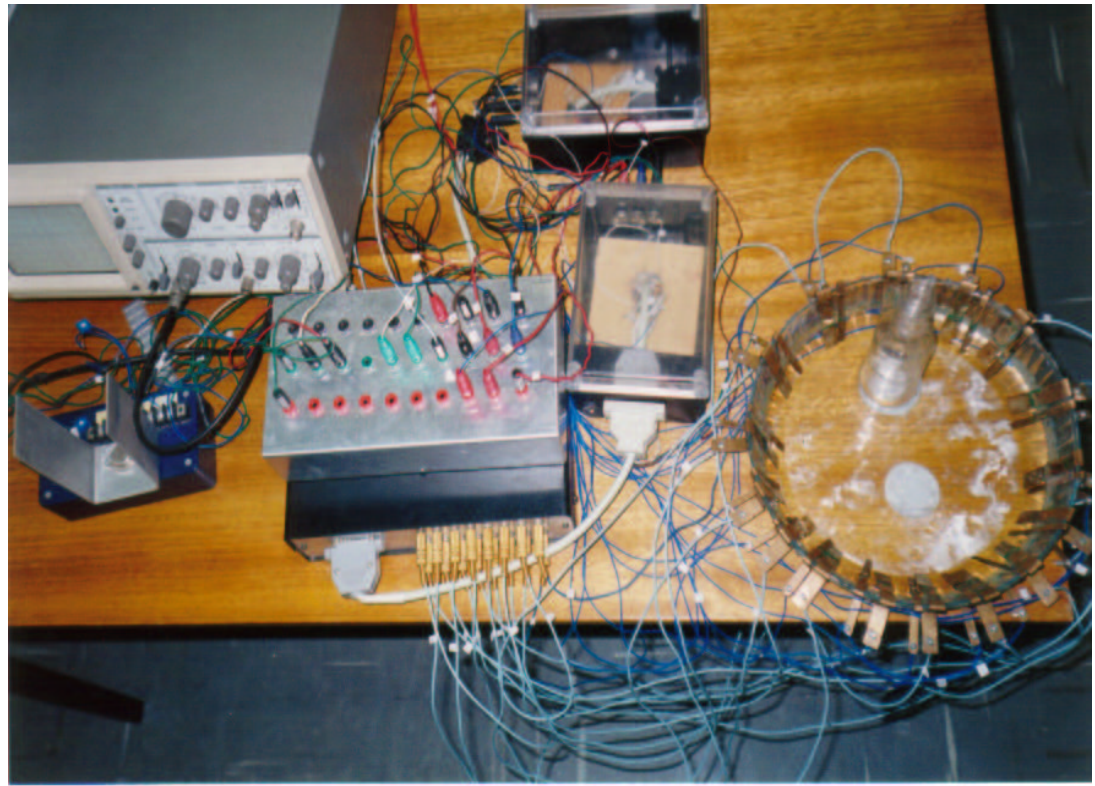

Figura 3.10: Segunda bancada B-2 de tomografia por impedância elétrica com demodulação analógica, conversor de voltagem para corrente e multiplexadores. 


\subsubsection{Bancada 3, B-3}

Uma terceira bancada de TIE foi desenvolvida, usando um conjunto de 32 eletrodos. O recipiente é simétrico, com as paredes verticais para fixar os eletrodos com parafusos, equi-espaçados ao redor do recipiente. Os novos eletrodos têm dimensões idênticas, condição que não foi imposta nos phantoms anteriores.

Esta bancada B-3 foi composta por :

- 32 eletrodos planos, de dimensões 6.0 x 0.9 x $0.1 \mathrm{~cm}$; construídos em chapa de bronze.

- recipiente de $22.0 \mathrm{~cm}$ de diâmetro, $5.5 \mathrm{~cm}$ de altura, fundo de vidro e paredes em PVC, como se mostra na Figura 3.11;

- solução salina com 1300 gramas de água destilada e 0.300 gramas de sal;

- objeto a estimar : cilíndrico, de vidro, com diâmetro de $6.1 \mathrm{~cm}$ e $13.0 \mathrm{~cm}$ de altura, colado com silicone no recipiente maior;

- padrão de corrente aplicado : diametral;

- fonte de corrente e gerador do sinal de fabricação alemã;

- fonte de alimentação : marca Power Supply, modelo MPC-3003D;

- multímetro de precisão, referência : HP34401 A, as medidas nesta bancada foram obtidas com a ajuda de um multímetro de precisão; a injeção de corrente e aquisição foram feitas manualmente;

- microcomputador, para arquivar os resultados. 


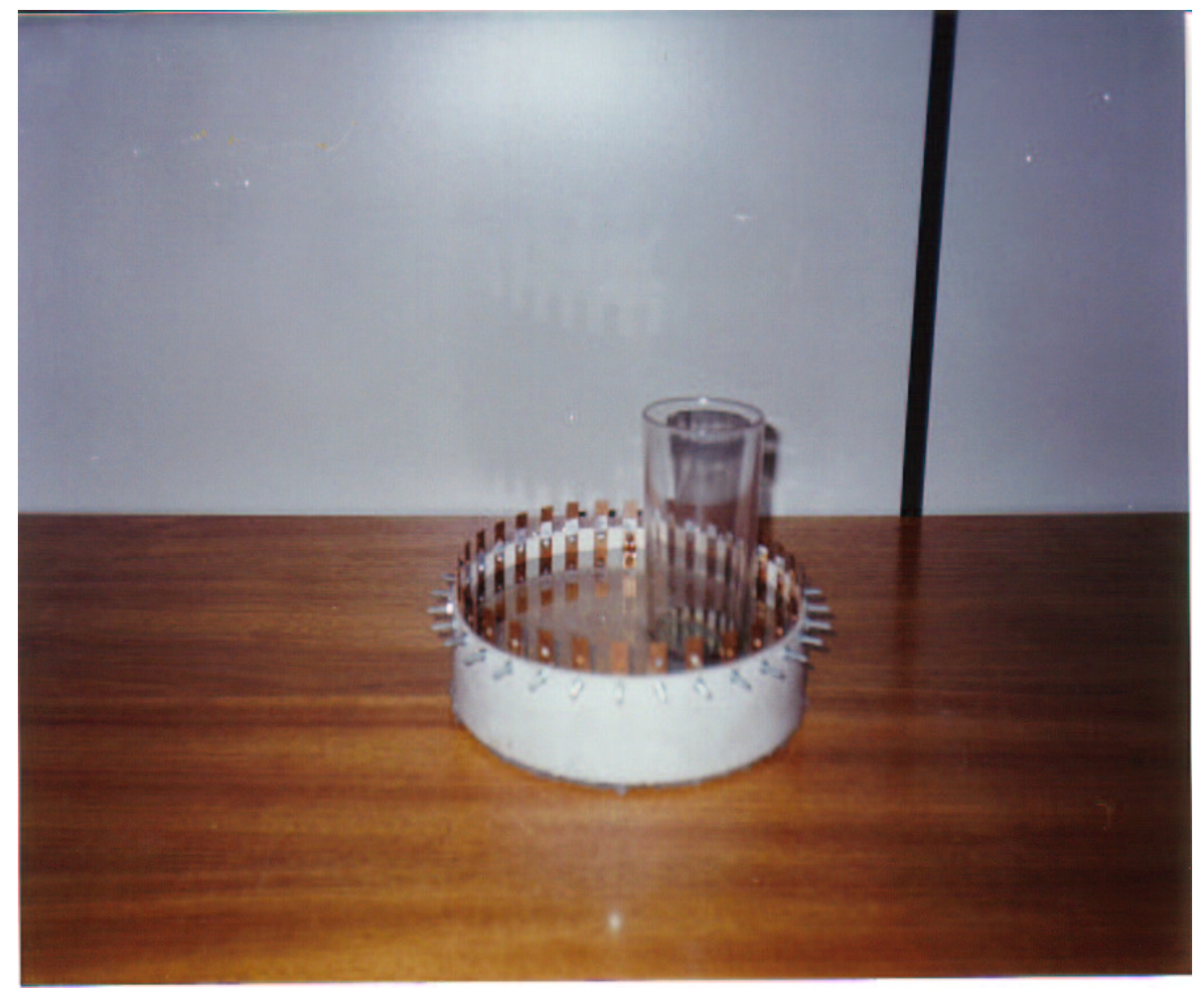

Figura 3.11: Phantom P-3 com solução salina, de diâmetro $22 \mathrm{~cm}$, com 32 eletrodos e objeto cilíndrico de vidro de diâmetro $6.1 \mathrm{~cm}$, da terceira bancada B-3. 


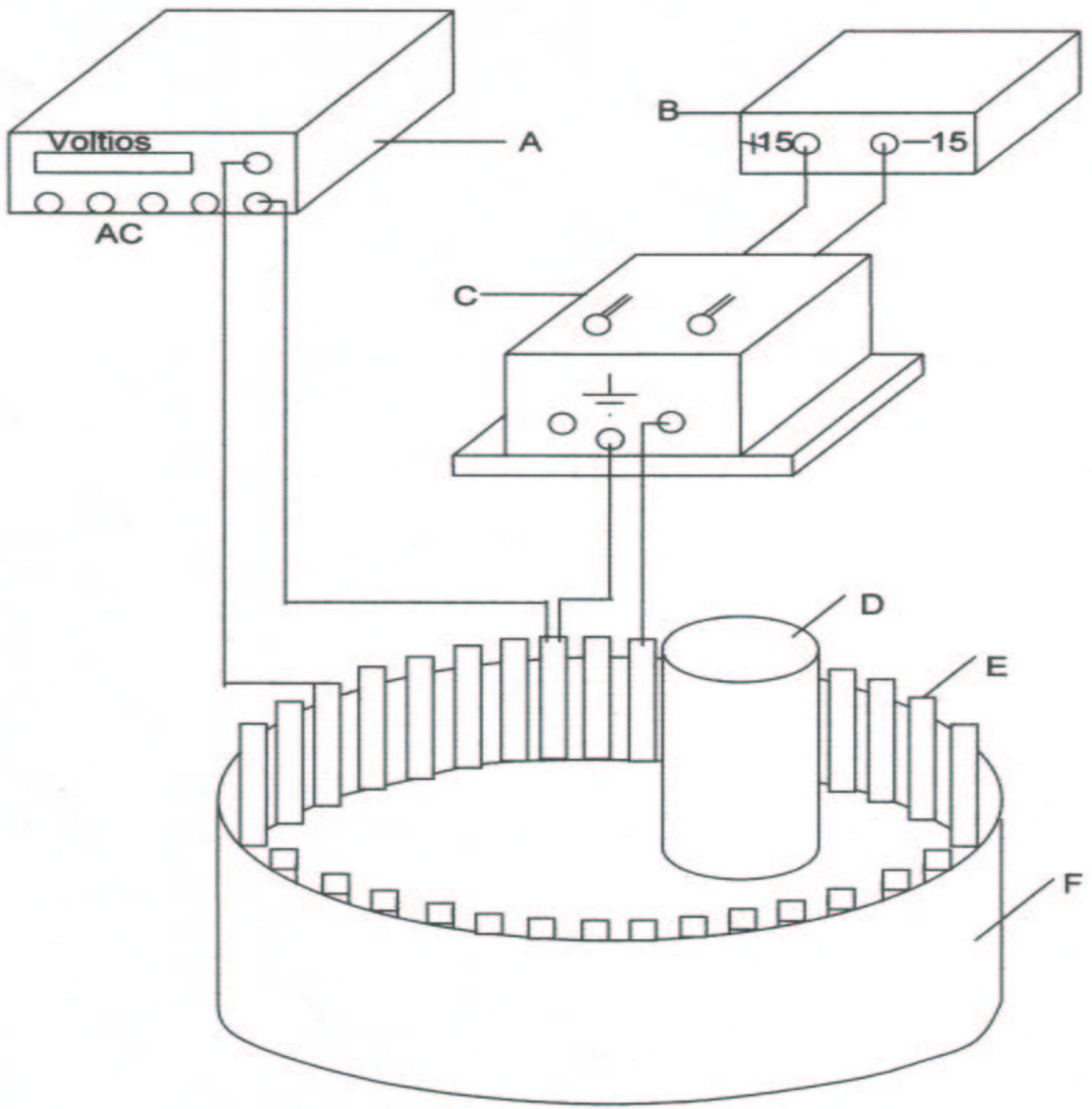

Figura 3.12: Montagem da terceira bancada B-3 de tomografia por impedância elétrica A. multímetro; B. fonte de alimentação; C. gerador de sinal e fonte de corrente; D. objeto cilíndrico de vidro; E. arranjo de eletrodos (32); F. recipiente com solução salina. 
Neste capítulo foram descritos as três bancadas nas quais foram sendo incluídas melhorias. Observou-se que a forma do recipiente, a geometria e a distribuição dos eletrodos nos phantoms tinham grande influência nos dados adquiridos.

Os dados experimentais utilizados neste trabalho foram obtidos através da bancada 3 . 


\section{Capítulo 4}

\section{METODOLOGIA PARA A SOLUÇÃO DO PROBLEMA DIRETO E INVERSO}

Neste capítulo, vamos definir o problema direto e o problema inverso em TIE. A solução do problema direto é utilizada iterativamente para resolver o problema inverso em alguns dos algoritmos de TIE.

No final do capítulo apresentam-se três exemplos e duas formas de solução para o problema inverso, numa malha de 4 elementos.

\subsection{Metodologia Para a Solução do Problema Direto}

O problema direto na TIE consiste em encontrar a distribuição do potencial elétrico na superfície de um objeto, conhecidas a distribuição de condutividades no domínio e a corrente no contorno.

Começa-se resolvendo um exemplo simples de duas dimensões, numa geometria circular conhecida com distribuição de condutividades homogêneas de $0.7(\Omega m)^{-1}$ e espessura de $0.027 \mathrm{~m}$, para o qual se quer conhecer as voltagens na superfície da geometria conforme a Figura 4.1.

Para a solução, utiliza-se a formulação de elementos finitos de duas dimensões, segundo Logan [25], o qual desenvolve as equações apropriadas.

\subsubsection{Discretização e Seleção do Tipo de Elemento}

Na solução de problemas de transferência de calor em duas dimensões, o elemento mais simples utilizado é o triangular com três nós [25]. Neste exemplo discretiza-se o domínio que representa a 


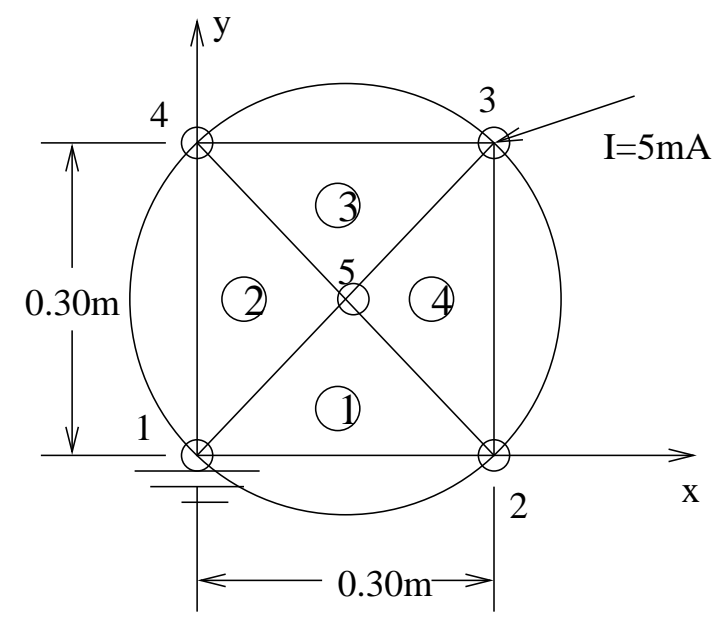

Figura 4.1: Malha de 4 elementos, 5 nós e 4 eletrodos.

seção do tórax em $n e=4$ elementos planos triangulares, $n n=5$ nós no total da malha e $n f=4$ eletrodos igualmente distribuídos, que são os 4 nós da fronteira do domínio. No nó 3 aplica-se uma corrente de $5 \mathrm{~mA}$, tendo como eletrodo de referência (ou terra) o eletrodo 1.

Para facilitar a discretização da geometria em elementos finitos triangulares, utiliza-se o módulo de geração automática de malhas Corduroy que faz parte do software FELt, o qual gera dois arquivos, um contendo a matriz das coordenadas $(x, y)$ dos nós, denominado $c$ d.dat e outro com a matriz das coordenadas dos três nós de cada um dos elementos da malha, denominado nodes.dat, assim :

$$
\begin{aligned}
\text { - o arquivo } c \text { d.dat contém a matriz }= & {\left[\begin{array}{ll}
x_{1} & y_{1} \\
x_{2} & y_{2} \\
x_{3} & y_{3} \\
x_{4} & y_{4} \\
x_{5} & y_{5}
\end{array}\right]=\left[\begin{array}{ll}
0 & 0 \\
0.30 & 0 \\
0.30 & 0.30 \\
0 & 0.30 \\
0.15 & 0.15
\end{array}\right] \text {; } } \\
\text { - o arquivo nodes.dat contém a matriz }= & {\left[\begin{array}{lll}
1 & 2 & 5 \\
1 & 5 & 4 \\
4 & 5 & 3 \\
2 & 3 & 5
\end{array}\right] . }
\end{aligned}
$$

\subsubsection{Cálculo da Matriz de Condutividade Local}

A matriz de condutividade local $y_{c}$, correspondente a cada elemento da malha, obtém-se a partir das seguintes equações do capítulo 13.6 de Logan [25] : 


$$
\left[y_{c}\right]=\iiint_{V} \frac{1}{4 \mathrm{~A}^{2}}[B]^{T}[D][B] d V=\frac{t \mathrm{~A}}{4 \mathrm{~A}^{2}}[B]^{T}[D][B]=\frac{t}{4 \mathrm{~A}}[B]^{T}[D][B]
$$

onde

$y_{c}$ : matriz de condutividade local para um elemento triangular;

$t$ : espessura igual a $0.027 \mathrm{~m}$;

$B$ : matriz da geometria de cada elemento, com função de interpolação linear;

$D$ : matriz com as propriedades do material, condutividade homogênea para os elementos;

$A$ : área de cada elemento triangular.

A matriz $[B]$ é dada por

$$
[B]=\left[\begin{array}{lll}
\beta_{i} & \beta_{j} & \beta_{m} \\
\gamma_{i} & \gamma_{j} & \gamma_{m}
\end{array}\right]
$$

com :

$$
\begin{aligned}
& \beta_{i}=y_{j}-y_{m}, \quad \beta_{j}=y_{m}-y_{i}, \quad \beta_{m}=y_{i}-y_{j} \\
& \gamma_{i}=x_{m}-x_{j}, \quad \gamma_{j}=x_{i}-x_{m}, \quad \gamma_{m}=x_{j}-x_{i}
\end{aligned}
$$

onde $x$ e $y$ são as coordenadas dos nós.

Agora, a matriz $[D]$ é dada por :

$$
[D]=\left[\begin{array}{cc}
\sigma_{x x} & 0 \\
0 & \sigma_{y y}
\end{array}\right]
$$

com : $\sigma_{x x}=\sigma_{y y}=0.7(\Omega m)^{-1}$, isto é, a condutividade é homogênea em cada elemento.

\section{Matriz de Condutividade Local do Elemento 1}

O cálculo da matriz de condutividade local do elemento 1 da malha de elementos finitos, começa com o uso da equação 4.3. A Figura 4.2 mostra as coordenadas $x$ e $y$ dos nós deste elemento.

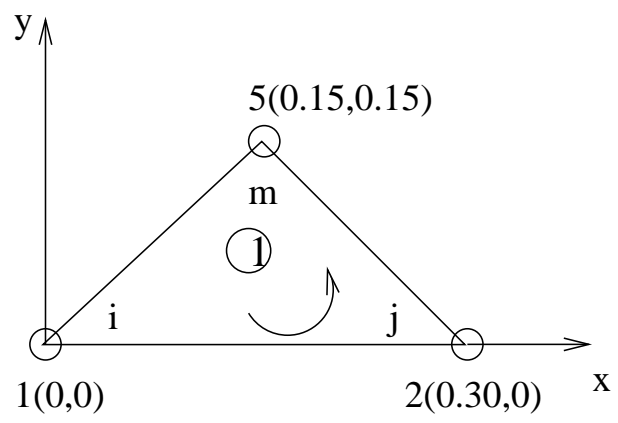

Figura 4.2: Elemento triangular 1 da malha de elementos finitos. 
Da equação 4.3 , resulta

$$
\begin{array}{ll}
\beta_{i}=0-0.15=-0.15, & \gamma_{i}=0.15-0.30=-0.15 \\
\beta_{j}=0.15-0=0.15, & \gamma_{j}=0-0.15=-0.15 \\
\beta_{m}=0-0=0, & \gamma_{m}=0.30-0=0.30
\end{array}
$$

Substituindo a equação 4.5 na equação 4.2 e o resultado obtido na equação 4.1, com a área do elemento 1 igual a $\mathrm{A}=\frac{0.30 * 0.15}{2}=0.0225$,

$$
\begin{aligned}
& \text { decorre } \\
& {\left[y_{c 1}\right]=\frac{0.027}{4 * 0.0225}\left[\begin{array}{rr}
-0.15 & -0.15 \\
0.15 & -0.15 \\
0 & 0.30
\end{array}\right]\left[\begin{array}{rr}
0.7 & 0 \\
0 & 0.7
\end{array}\right]\left[\begin{array}{rrr}
-0.15 & 0.15 & 0 \\
-0.15 & -0.15 & 0.30
\end{array}\right]} \\
& 125 \\
& {\left[y_{c 1}\right]=\left[\begin{array}{rrr}
0.00945 & 0 & -0.00945 \\
0 & 0.00945 & -0.00945 \\
-0.00945 & -0.00945 & 0.0189
\end{array}\right]}
\end{aligned}
$$

onde os valores 1,2 e 5 fazem referência aos nós $i, j$ e $m$ do elemento 1 , de acordo com a primeira linha da matriz nodes.dat.

\section{Matriz de Condutividade Local do Elemento 2}

De forma similar, o cálculo da matriz de condutividade local do elemento 2 começa com o uso da equação 4.3. A Figura 4.3 mostra as coordenadas $x$ e $y$ dos nós deste elemento.

Da equação 4.3 , resulta

$$
\begin{array}{ll}
\beta_{i}=0.15-0.30=-0.15, & \gamma_{i}=0-0.15=-0.15 \\
\beta_{j}=0.30-0=0.30, & \gamma_{j}=0-0=0 \\
\beta_{m}=0-0.15=-0.15, & \gamma_{m}=0.15-0=0.15
\end{array}
$$

Substituindo a equação 4.7 na equação 4.2 e o resultado obtido na equação 4.1 , com a área do elemento 2 dada por : $\mathrm{A}=\frac{0.30 * 0.15}{2}=0.0225$,

decorre

$$
\left[y_{c 2}\right]=\frac{0.027}{4 * 0.0225}\left[\begin{array}{rr}
-0.15 & -0.15 \\
0.30 & 0 \\
-0.15 & 0.15
\end{array}\right]\left[\begin{array}{rr}
0.7 & 0 \\
0 & 0.7
\end{array}\right]\left[\begin{array}{rrr}
-0.15 & 0.30 & -0.15 \\
-0.15 & 0 & 0.15
\end{array}\right]
$$




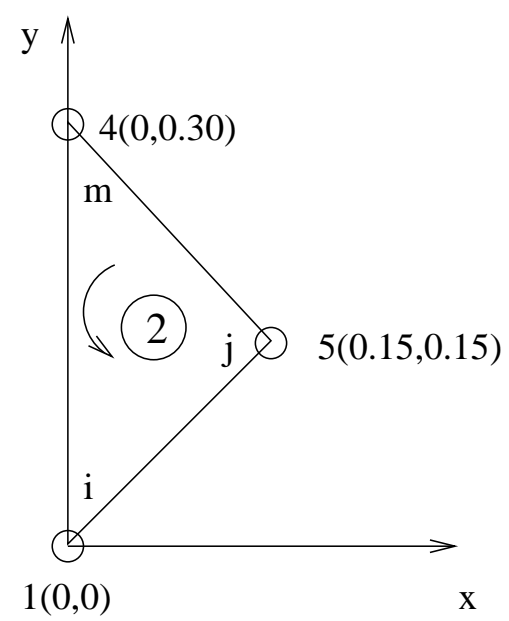

Figura 4.3: Elemento triangular 2 da malha de elementos finitos.

$$
\left[y_{c 2}\right]=\left[\begin{array}{rrr}
1 & 5 & \multicolumn{1}{c}{4} \\
0.00945 & -0.00945 & 0 \\
-0.00945 & 0.0189 & -0.00945 \\
0 & -0.00945 & 0.00945
\end{array}\right]
$$

onde os valores 1,5 e 4 fazem referência aos nós $i, j$ e $m$ do elemento 2 , de acordo com a segunda linha da matriz nodes.dat.

\section{Matriz de Condutividade Local do Elemento 3}

Na Figura 4.4 observam-se as coordenadas $x$ e $y$ dos nós deste elemento.

Para o cálculo da matriz local do elemento 3, resulta da equação 4.3 :

$$
\begin{array}{ll}
\beta_{i}=0.15-0.30=-0.15, & \gamma_{i}=0.30-0.15=0.15 \\
\beta_{j}=0.30-0.30=0, & \gamma_{j}=0-0.30=-0.30 \\
\beta_{m}=0.30-0.15=0.15, & \gamma_{m}=0.15-0=0.15
\end{array}
$$

Substituindo a equação 4.9 na equação 4.2 e o resultado obtido na equação 4.1, com a área do elemento 3 igual a $A=\frac{0.30 * 0.15}{2}=0.0225$,

decorre

$$
\left[y_{c 3}\right]=\frac{0.027}{4 * 0.0225}\left[\begin{array}{rr}
-0.15 & 0.15 \\
0 & -0.30 \\
0.15 & 0.15
\end{array}\right]\left[\begin{array}{rr}
0.7 & 0 \\
0 & 0.7
\end{array}\right]\left[\begin{array}{rrr}
-0.15 & 0 & 0.15 \\
0.15 & -0.30 & 0.15
\end{array}\right]
$$




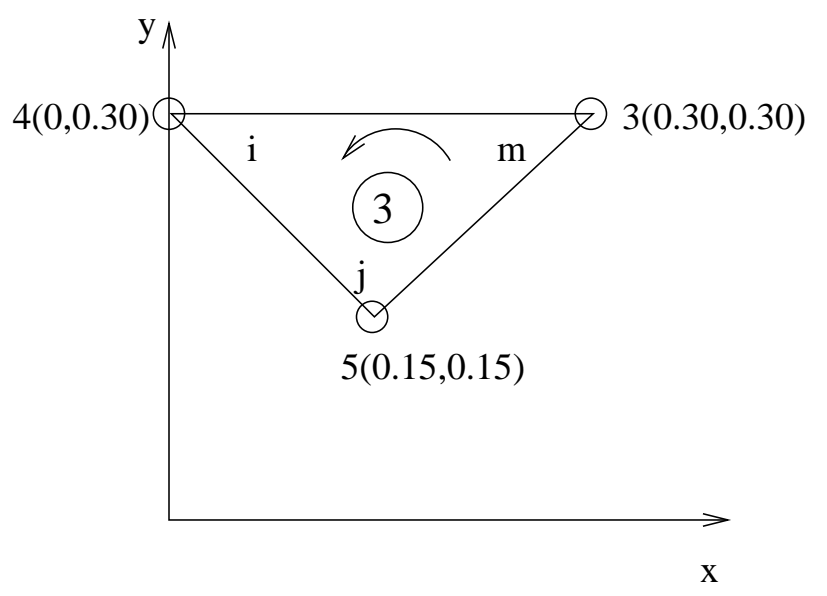

Figura 4.4: Elemento triangular 3 da malha de elementos finitos.

$$
\left[y_{c 3}\right]=\left[\begin{array}{rrr}
4 & 5 & 3 \\
0.00945 & -0.00945 & 0 \\
-0.00945 & 0.0189 & -0.00945 \\
0 & -0.00945 & 0.00945
\end{array}\right]
$$

onde os valores 4,5 e 3 fazem referência aos nós $i, j$ e $m$ do elemento 3 , de acordo com a terceira linha da matriz nodes.dat.

\section{Matriz de Condutividade Local do Elemento 4}

De forma similar, o cálculo da matriz de condutividade local do elemento 4 começa com o uso da equação 4.3. A Figura 4.5 mostra as coordenadas $x$ e $y$ dos nós deste elemento.

$$
\begin{array}{ll}
\beta_{i}=0.30-0.15=0.15, & \gamma_{i}=0.15-0.30=-0.15 \\
\beta_{j}=0.15-0=0.15, & \gamma_{j}=0.30-0.15=0.15 \\
\beta_{m}=0-0.30=-0.30, & \gamma_{m}=0.30-0.30=0
\end{array}
$$

Substituindo a equação 4.11 na equação 4.2 e o resultado obtido na equação 4.1, com a área do elemento 4 dada por : $\mathrm{A}=\frac{0.30 * 0.15}{2}=0.0225$,

resulta

$$
\left[y_{c 4}\right]=\frac{0.027}{4 * 0.0225}\left[\begin{array}{rr}
0.15 & -0.15 \\
0.15 & 0.15 \\
-0.30 & 0
\end{array}\right]\left[\begin{array}{rr}
0.7 & 0 \\
0 & 0.7
\end{array}\right]\left[\begin{array}{rrr}
0.15 & 0.15 & -0.30 \\
-0.15 & 0.15 & 0
\end{array}\right]
$$




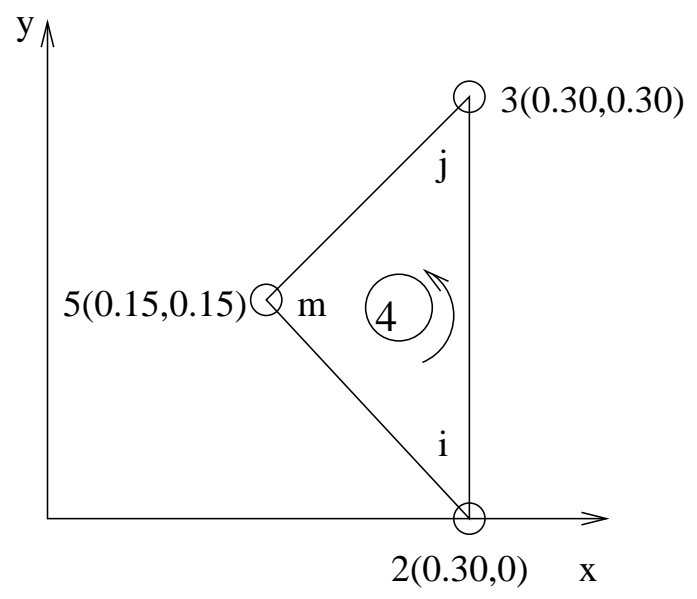

Figura 4.5: Elemento triangular 4 da malha de elementos finitos.

$$
\left[y_{c 4}\right]=\left[\begin{array}{rrr}
2 & 3 & 5 \\
0.00945 & 0 & -0.00945 \\
0 & 0.00945 & -0.00945 \\
-0.00945 & -0.00945 & 0.0189
\end{array}\right]
$$

onde os valores 2, 3 e 5 fazem referência aos nós $i, j$ e $m$ do elemento 4 , de acordo com a quarta linha da matriz nodes.dat.

\subsubsection{Matriz de Condutividade Global}

Cada linha e cada coluna da matriz de condutividade global está associado a um particular nó. Cada matriz de condutividade local pode ser ampliada para incluir colunas e linhas associadas a todos os nós da malha. A soma de todas as matrizes de condutividade locais ampliadas é a matriz de condutividade global. Neste exemplo a matriz de condutividade global é.

$$
[Y]=\left[\begin{array}{rrrrc}
1 & 2 & 3 & 4 & 5 \\
0.0189 & 0 & 0 & 0 & -0.0189 \\
0 & 0.0189 & 0 & 0 & -0.0189 \\
0 & 0 & 0.0189 & 0 & -0.0189 \\
0 & 0 & 0 & 0.0189 & -0.0189 \\
-0.0189 & -0.0189 & -0.0189 & -0.0189 & 0.0756
\end{array}\right]
$$

Observa-se que a matriz de condutividade global $Y$ é uma matriz simétrica de dimensão 5x5, ou seja, número de nós da malha por número de nós da malha $\left(n n_{x} n n\right)$. 


\subsubsection{Cálculo das Voltagens}

Para o cálculo final das voltagens, utiliza-se a expressão

$$
[Y] *\left[V_{i}\right]=\left[C_{j}\right]
$$

onde

$[Y]$ : matriz de condutividade global, matriz conhecida da expressão 4.13;

$\left[V_{i}\right]$ : vetor de voltagens que serão calculados, voltagens correspondentes aos 5 nós. $i=$ $1, . ., n n ;$ com $n n$ igual ao número de nós;

$\left[C_{j}\right]$ : vetor de correntes ou padrão de corrente, onde $j=1, \ldots p$ e $p$ é o número de padrões de corrente; neste caso como foi aplicada uma corrente de $5 m A$ ao nó 3 , o vetor de correntes $\left[C_{2}\right]$ equivalente é :

$$
\left[C_{2}\right]=\left[\begin{array}{r}
0 \\
0 \\
0.005 \\
0 \\
0
\end{array}\right]
$$

Obtém-se da equação 4.14 :

$$
\left[V_{i}\right]=\left[Y^{-1}\right] *\left[C_{j}\right]
$$

onde $\left[Y^{-1}\right]$ : é a inversa da matriz de condutividade global. O cálculo de $Y^{-1}$ envolve a eliminação da singularidade da matriz $Y$ que é feita com a introdução das condições de contorno, entre as quais pode-se impor a voltagem de um dos eletrodos igual a zero, voltagem de referência. Atribuindo-se o valor unitário ao elemento $(1,1)$ da matriz de condutividade global e igualando a zero os demais elementos na primeira fila e na primeira coluna, tem-se :

$$
[Y]=\left[\begin{array}{rrrrr}
1 & 0 & 0 & 0 & 0 \\
0 & 0.0189 & 0 & 0 & -0.0189 \\
0 & 0 & 0.0189 & 0 & -0.0189 \\
0 & 0 & 0 & 0.0189 & -0.0189 \\
0 & -0.0189 & -0.0189 & -0.0189 & 0.0756
\end{array}\right]
$$

a sua inversa é dada por : 


$$
\left[Y^{-1}\right]=\left[\begin{array}{rrrrr}
1.00000 & 0.00000 & 0.00000 & 0.00000 & 0.00000 \\
0.00000 & 105.82011 & 52.91005 & 52.91005 & 52.91005 \\
0.00000 & 52.91005 & 105.82011 & 52.91005 & 52.91005 \\
0.00000 & 52.91005 & 52.91005 & 105.82011 & 52.91005 \\
0.00000 & 52.91005 & 52.91005 & 52.91005 & 52.91005
\end{array}\right]
$$

então, a solução da equação 4.16 é igual a

$$
\left[V_{i}\right]=\left[Y^{-1}\right] *\left[C_{2}\right]=\left[\begin{array}{c}
0.00000 \\
0.26455 \\
0.52910 \\
0.26455 \\
0.26455
\end{array}\right]
$$

Esta é a solução do problema direto da TIE. O vetor $\left[V_{i}\right]$ contém as voltagens de todos os nós da malha, sendo o valor zero para o nó 1 que é o nó de referência (ou terra) o valor máximo da voltagem é o do nó 3 onde foi injetada a corrente. As voltagens $v_{1}, v_{2}, v_{3}, v_{4}$ são as correspondentes aos $n f$ eletrodos.

Desenvolveu-se um algoritmo no software Octave para o cálculo do problema direto, o qual com os dados do exemplo gera o resultado mostrado na Figura 4.6.

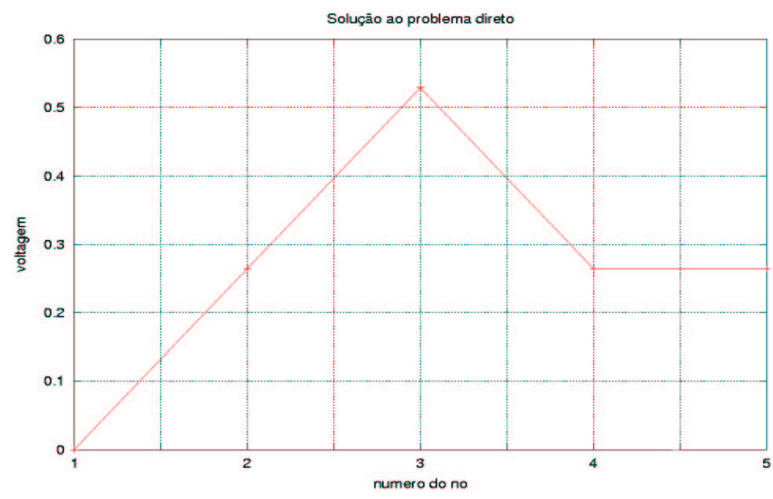

Figura 4.6: Cálculo das voltagens dos nós no problema direto.

\subsection{Metodologia Para a Solução do Problema Inverso}

O problema inverso consiste na obtenção da distribuição de condutividades na seção do corpo em estudo, conhecidas da distribuição de potencial (ou voltagens medidas) e da corrente injetada na superfície. 
Observe o exemplo estudado no problema direto. Considerando desconhecidas as condutividades dos 4 elementos da malha de elementos finitos, pode-se estabelecer uma metodologia para sua solução.

O primeiro passo é gerar a malha e selecionar o tipo de elemento. Utilizam-se a mesma malha e o mesmo tipo de elemento do exemplo anterior.

\subsubsection{Cálculo da Matriz de Condutividade Local}

A matriz de condutividade local para cada elemento da malha é obtida a partir da equação 4.1, a qual fica em função da condutividade de cada elemento.

\section{Matriz de Condutividade Local do Elemento 1}

A matriz de condutividade local do elemento 1 é obtida substituindo a equação 4.5 na equação 4.2 e a equação 4.2 na equação 4.1. Assim, utilizando os dados numéricos, escreve-se

$$
\left[y_{c 1}\right]=\frac{0.027}{4 * 0.0225}\left[\begin{array}{rr}
-0.15 & -0.15 \\
0.15 & -0.15 \\
0 & 0.30
\end{array}\right]\left[\begin{array}{rr}
\sigma_{1} & 0 \\
0 & \sigma_{1}
\end{array}\right]\left[\begin{array}{rrr}
-0.15 & 0.15 & 0 \\
-0.15 & -0.15 & 0.30
\end{array}\right]
$$

onde $\sigma_{1}$ é a condutividade do elemento 1 .

A matriz $\left[y_{c 1}\right]$ resulta

$$
\left[y_{c 1}\right]=\left[\begin{array}{rrr}
1 & 2 & \multicolumn{1}{c}{5} \\
0.0135 * \sigma_{1} & 0 & -0.0135 * \sigma_{1} \\
0 & 0.0135 * \sigma_{1} & -0.0135 * \sigma_{1} \\
-0.0135 * \sigma_{1} & -0.0135 * \sigma_{1} & 0.027 * \sigma_{1}
\end{array}\right]
$$

A qual é denominada matriz de condutividade local simbólica do elemento 1, por estar em função da condutividade do elemento $1\left(\sigma_{1}\right)$.

\section{Matriz de Condutividade Local do Elemento 2}

A matriz de condutividade local do elemento 2 é obtida substituindo a equação 4.7 na equação 4.2 e a equação 4.2 na equação 4.1. Assim, utilizando os dados numéricos, escreve-se

$$
\left[y_{c 2}\right]=\frac{0.027}{4 * 0.0225}\left[\begin{array}{rr}
-0.15 & -0.15 \\
0.30 & 0 \\
-0.15 & 0.15
\end{array}\right]\left[\begin{array}{rr}
\sigma_{2} & 0 \\
0 & \sigma_{2}
\end{array}\right]\left[\begin{array}{rrr}
-0.15 & 0.30 & -0.15 \\
-0.15 & 0 & 0.15
\end{array}\right]
$$

onde $\sigma_{2}$ é a condutividade do elemento 2 . 
A matriz $\left[y_{c 2}\right]$ resulta

$$
\left[y_{c 2}\right]=\left[\begin{array}{rrr}
1 & 5 & \multicolumn{1}{c}{4} \\
0.0135 * \sigma_{2} & -0.0135 * \sigma_{2} & 0 \\
-0.0135 * \sigma_{2} & 0.027 * \sigma_{2} & -0.0135 * \sigma_{2} \\
0 & -0.0135 * \sigma_{2} & 0.0135 * \sigma_{2}
\end{array}\right]
$$

A matriz 4.21 é denominada matriz de condutividade local simbólica do elemento 2, por estar em função da condutividade do elemento $2\left(\sigma_{2}\right)$.

\section{Matriz de Condutividade Local do Elemento 3}

De forma similar calcula-se a matriz de condutividade local do elemento 3. Substituindo a equação 4.9 na equação 4.2 e a equação 4.2 na equação 4.1 , e usando os dados numéricos, escreve-se

$$
\left[y_{c 3}\right]=\frac{0.027}{4 * 0.0225}\left[\begin{array}{rr}
-0.15 & 0.15 \\
0 & -0.30 \\
0.15 & 0.15
\end{array}\right]\left[\begin{array}{rr}
\sigma_{3} & 0 \\
0 & \sigma_{3}
\end{array}\right]\left[\begin{array}{rrr}
-0.15 & 0 & 0.15 \\
0.15 & -0.30 & 0.15
\end{array}\right]
$$

onde $\sigma_{3}$ é a condutividade do elemento 3 .

A matriz $\left[y_{c 3}\right]$ resulta

$$
\left[y_{c 3}\right]=\left[\begin{array}{rrr}
4 & 5 & 3 \\
0.0135 * \sigma_{3} & -0.0135 * \sigma_{3} & 0 \\
-0.0135 * \sigma_{3} & 0.027 * \sigma_{3} & -0.0135 * \sigma_{3} \\
0 & -0.0135 * \sigma_{3} & 0.0135 * \sigma_{3}
\end{array}\right]
$$

A matriz 4.22 é denominada matriz de condutividade local simbólica do elemento 3, por estar em função da condutividade do elemento $3\left(\sigma_{3}\right)$.

\section{Matriz de Condutividade Local do Elemento 4}

A matriz de condutividade local do elemento 4 obtém-se substituindo a equação 4.11 na equação 4.2 e a equação 4.2 na equação 4.1 , e utilizando os dados numéricos, escreve-se

$$
\left[y_{c 4}\right]=\frac{0.027}{4 * 0.0225}\left[\begin{array}{rr}
0.15 & -0.15 \\
0.15 & 0.15 \\
-0.30 & 0
\end{array}\right]\left[\begin{array}{rr}
\sigma_{4} & 0 \\
0 & \sigma_{4}
\end{array}\right]\left[\begin{array}{rrr}
0.15 & 0.15 & -0.30 \\
-0.15 & 0.15 & 0
\end{array}\right]
$$

onde $\sigma_{4}$ é a condutividade do elemento 4 .

A matriz $\left[y_{c 4}\right]$ resulta

$$
\left[y_{c 4}\right]=\left[\begin{array}{rrr}
2 & 3 & 5 \\
0.0135 * \sigma_{4} & 0 & -0.0135 * \sigma_{4} \\
0 & 0.0135 * \sigma_{4} & -0.0135 * \sigma_{4} \\
-0.0135 * \sigma_{4} & -0.0135 * \sigma_{4} & 0.027 * \sigma_{4}
\end{array}\right]
$$


A matriz 4.23 é denominada matriz de condutividade local simbólica do elemento 4, por estar em função da condutividade do elemento $4\left(\sigma_{4}\right)$.

\subsubsection{Matriz de Condutividade Global Simbólica}

A partir das matrizes de condutividade locais simbólicas de cada um dos elementos, elemento 1: matriz (4.20), elemento 2: matriz (4.21), elemento 3: matriz (4.22) e elemento 4: matriz (4.23), somam-se os respectivos elementos das linhas e colunas de cada um dos nós, resultando a matriz denominada matriz de condutividade global simbólica $Y$.

$$
[Y]=\left[\begin{array}{rrrrr}
0.0135\left(\sigma_{1}+\sigma_{2}\right) & 0 & 0 & 0 & -0.0135\left(\sigma_{1}+\sigma_{2}\right) \\
0 & 0.0135\left(\sigma_{1}+\sigma_{4}\right) & 0 & 0 & -0.0135\left(\sigma_{1}+\sigma_{4}\right) \\
0 & 0 & 0.0135\left(\sigma_{3}+\sigma_{4}\right) & 0 & -0.0135\left(\sigma_{3}+\sigma_{4}\right) \\
0 & 0 & 0 & 0.0135\left(\sigma_{2}+\sigma_{3}\right) & -0.0135\left(\sigma_{2}+\sigma_{3}\right) \\
-0.0135\left(\sigma_{1}+\sigma_{2}\right) & -0.0135\left(\sigma_{1}+\sigma_{4}\right) & -0.0135\left(\sigma_{3}+\sigma_{4}\right) & -0.0135\left(\sigma_{2}+\sigma_{3}\right) & 0.027\left(\sigma_{1}+\sigma_{2}+\sigma_{3}+\sigma_{4}\right)
\end{array}\right]
$$

A matriz 4.24 é denominada matriz de condutividade global simbólica, por estar em função das condutividades do elemento $1\left(\sigma_{1}\right)$, do elemento $2\left(\sigma_{2}\right)$, do elemento $3\left(\sigma_{3}\right)$ e do elemento 4 $\left(\sigma_{4}\right)$.

Observa-se que a matriz de condutividade global simbólica, é uma matriz simétrica de dimensão $5 \times 5,\left(n n_{x} n n\right)$ onde $n n$ é o número de nós da malha.

Para o cálculo da inversa da nova matriz 4.24, é preciso estimar valores iniciais para as condutividades de cada um dos elementos e eliminar a singularidade da mesma forma que no problema direto.

As voltagens são calculadas através da equação 4.16, diversas vezes, alterando os valores das condutividades, até obter uma estimativa da distribuição de condutividades que minimizem a diferença entre os potenciais medidos e os calculados. Entre os métodos para a solução do chamado problema inverso encontram-se a abordagem clássica relativa [32], a abordagem dinâmica e o método de Newton-Raphson.

\subsection{Metodologia Para a Solução do Problema Inverso, Aplicando o Filtro de Kalman}

Vauhkonen [35, 36] abordou o problema inverso como um problema de estimação de estados, utilizando o filtro linearizado de Kalman. Uma nova estimativa da distribuição de condutividades foi obtida, após ser aplicado cada padrão de corrente. A seguir descreve-se a metodologia para implementar esta solução no exemplo da Figura 4.1. 


\subsubsection{Matriz de Sensibilidade $H$}

O método do Filtro de Kalman é baseado na matriz de sensibilidade [32, 39].

Define-se a matriz de sensibilidade como $H_{j}\left(\sigma_{0}\right)$ :

$$
H_{j}\left(\sigma_{o}\right)=\frac{\partial\left[Y(\sigma)^{-1} c_{j}\right]}{\partial \sigma} .
$$

onde $c_{j}$ denota um padrão de excitação e $Y$ é a matriz de condutividade.

Conhece-se a equação 4.26 .

$$
Y Y^{-1}=I
$$

Deriva-se a equação 4.26 ,

$$
\frac{\partial Y}{\partial \sigma} Y^{-1}+Y \frac{\partial Y^{-1}}{\partial \sigma}=0
$$

E ordena-se a equação 4.27,

$$
Y \frac{\partial Y^{-1}}{\partial \sigma}=-\frac{\partial Y}{\partial \sigma} Y^{-1}
$$

Multiplicando ambos os lados da equação 4.28 por $Y^{-1}$

$$
Y^{-1} Y \frac{\partial Y^{-1}}{\partial \sigma}=-Y^{-1} \frac{\partial Y}{\partial \sigma} Y^{-1}
$$

e substituindo a equação 4.26 na equação 4.29 , resulta

$$
\frac{\partial Y^{-1}}{\partial \sigma}=-Y^{-1} \frac{\partial Y}{\partial \sigma} Y^{-1} .
$$

Finalmente, substituindo a equação 4.30 na equação 4.25 que define a matriz de sensibilidade,

$$
H_{j, i}\left(\sigma_{o}\right)=-Y^{-1} \frac{\partial Y}{\partial \sigma_{i}} Y^{-1} c_{j}
$$

onde

$H_{j, i}\left(\sigma_{o}\right)$ : matriz de sensibilidade em função das condutividades dos elementos $\sigma_{i}$, com $j=$ $1, \ldots . p$, sendo $p$ o número de padrões de corrente;

$Y^{-1}$ : inversa da matriz de condutividade global simbólica;

$\frac{\partial Y}{\partial \sigma_{i}}$ : derivada parcial da matriz de condutividade simbólica com respeito à condutividade de um dos elementos, com $i=1, \ldots . . n e$, sendo ne o número de elementos;

$c_{j}$ : padrão de corrente injetado; $j=1, . ., p$, sendo $p$ o número de padrões de corrente.

A equação 4.25 torna-se 


$$
H_{j}\left(\sigma_{o}\right)=\left[\begin{array}{llll}
-Y^{-1} \frac{\partial Y}{\partial \sigma_{1}} Y^{-1} c_{j} & -Y^{-1} \frac{\partial Y}{\partial \sigma_{2}} Y^{-1} c_{j} & \ldots & -Y^{-1} \frac{\partial Y}{\partial \sigma_{n e}} Y^{-1} c_{j}
\end{array}\right] .
$$

Para o exemplo da Figura 4.1, a matriz de sensibilidade $H_{j}(\sigma)$ é dada por :

$$
H_{j}(\sigma)=\left[\begin{array}{llll}
-Y^{-1} \frac{\partial Y}{\partial \sigma_{1}} Y^{-1} c_{j} & -Y^{-1} \frac{\partial Y}{\partial \sigma_{2}} Y^{-1} c_{j} & -Y^{-1} \frac{\partial Y}{\partial \sigma_{3}} Y^{-1} c_{j} & -Y^{-1} \frac{\partial Y}{\partial \sigma_{4}} Y^{-1} c_{j}
\end{array}\right]
$$

\subsubsection{Equações do Filtro de Kalman}

O Filtro de Kalman requer o modelo de observação e o modelo do sistema na forma de espaço de estados. Define-se o modelo de observação [32]

$$
v_{k}\left(\sigma_{k}\right)=v_{k}\left(\sigma_{k-1}\right)+\left[H_{k}\left(\sigma_{k-1}\right)\right]\left[\sigma_{k}-\sigma_{k-1}\right]+\nu_{k}
$$

onde $v_{k}$ é o vetor de voltagens nos eletrodos, $\sigma_{k}$ representa a distribuição de condutividades, $H$ é a matriz de sensibilidade e $\nu_{k}$ é o vetor onde cada elemento procede de uma sequiência de ruído branco Gaussiano de média nula que corrompe as medidas e cuja matriz de covariância é dada por $R_{k}$.

Define-se o modelo do sistema ou equação de estado [32]

$$
\sigma_{k}=\Phi_{k-1} \sigma_{k-1}+\omega_{k-1}
$$

onde $\Phi_{k-1}$ é a matriz de transição de estados e $\omega_{k-1}$ é o vetor onde cada elemento procede de uma sequiência de ruído branco Gaussiano de média nula que corrompe o estado e cuja matriz de covariância é dada por $Q_{k}$.

No Apêndice A desenvolvem-se as equações recursivas do Filtro de Kalman que permitem a solução do problema inverso. O seu desenvolvimento baseia-se nos trabalhos de Kalman [22, 23] e Brown [8].

O Filtro de Kalman consiste das seguintes equações (equações recursivas ) :

- equações de propagação

propagação de estado :

$$
\hat{\sigma}_{k}^{(-)}=\Phi_{k-1} \hat{\sigma}_{k-1}^{(+)}
$$

propagação da covariância de estado :

$$
P_{k}^{(-)}=\Phi_{k-1} P_{k-1}^{(+)} \Phi_{k-1}^{t}+Q_{k-1}
$$


- equações de atualização

ganho do filtro :

$$
G_{k}=P_{k}^{(-)} H_{k}^{(n f) t}\left(\hat{\sigma}_{k}^{(-)}\right)\left[H_{k}^{(n f)}\left(\hat{\sigma}_{k}^{(-)}\right) P_{k}^{(-)} H_{k}^{(n f) t}\left(\hat{\sigma}_{k}^{(-)}\right)+R_{k}\right]^{-1}
$$

atualização da matriz de covariância :

$$
P_{k}^{(+)}=\left[I-G_{k} H_{k}^{(n f)}\left(\hat{\sigma}_{k}^{(-)}\right)\right] P_{k}^{(-)}
$$

atualização do estado :

$$
\hat{\sigma}_{k}^{(+)}=\hat{\sigma}_{k}^{(-)}+G_{k}\left[v_{k}^{(n f)}-h_{k}^{(n f)}\left(\hat{\sigma}_{k}^{(-)}\right)\right]
$$

\subsubsection{Condição Inicial do Vetor $\hat{\sigma}_{o}^{(+)}$}

Admitindo-se conhecida a condição inicial do vetor de condutividades $\hat{\sigma}_{o}^{(+)}$, ou seja, $\sigma(t)$ em $t=0$. O vetor $\hat{\sigma}_{o}^{(+)}$tem dimensão $\left(n e_{x} 1\right)$.

Considere a condutividade inicial homogênea igual a $0.3(\Omega m)^{-1}$ no exemplo com 4 elementos, então

$$
\hat{\sigma}_{o}^{(+)}=\left[\begin{array}{c}
0.3 \\
0.3 \\
0.3 \\
0.3
\end{array}\right]
$$

$\mathrm{O}$ vetor $\hat{\sigma}_{o}^{(+)}$é utilizado somente na primeira iteração do Filtro de Kalman. Não é necessário conhecimento prévio acerca da distribuição inicial de condutividades.

\subsubsection{Condição Inicial da Matriz $P_{o}^{(+)}$}

A condição inicial da matriz de covariância do erro de estimação, $P_{o}^{(+)}$é, por simplicidade, uma matriz diagonal com elementos iguais entre si e de dimensão $\left(n e_{x} n e\right)$.

$$
P_{o}^{(+)}=\left[\begin{array}{rrrr}
5 * 10^{-2} & 0 & 0 & 0 \\
0 & 5 * 10^{-2} & 0 & 0 \\
0 & 0 & 5 * 10^{-2} & 0 \\
0 & 0 & 0 & 5 * 10^{-2}
\end{array}\right] .
$$

O valor dos elementos da diagonal foi definido por experimentação numérica. Após algumas iterações, o Filtro de Kalman ajusta as matrizes $P_{k}^{(-)}$subseqüentes. 


\subsubsection{Cálculo do Vetor $\hat{\sigma}_{k}^{(-)}$}

O cálculo do vetor de propagação da estimativa do estado $\hat{\sigma}_{k}^{(-)}$baseia-se na equação 4.36.

A matriz de transição procura incorporar um modelo matemático com a dinâmica real do sistema. Porém pela complexidade das estruturas que compõem o tórax e à ausência de modelos fidedignos, optou-se pela formulação proposta por Vauhkonen et al. [36], em que a matriz de transição de estado $\Phi_{k-1}$ é a matriz identidade de dimensão $\left(n e_{x} n e\right)$ :

$$
\Phi_{k-1}=\left[\begin{array}{cccc}
1 & 0 & 0 & 0 \\
0 & 1 & 0 & 0 \\
0 & 0 & 1 & 0 \\
0 & 0 & 0 & 1
\end{array}\right]
$$

Em forma geral, explicitando as dimensões das matrizes em 4.36, tem-se :

$\hat{\sigma}_{k\left(n e_{x} 1\right)}^{(-)}=\Phi_{k-1\left(n e_{x} n e\right)} \hat{\sigma}_{k-1\left(n e_{x} 1\right)}^{(+)}$

Logo, para o exemplo de 4 elementos,

$$
\hat{\sigma}_{k}^{(-)}=\left[\begin{array}{cccc}
1 & 0 & 0 & 0 \\
0 & 1 & 0 & 0 \\
0 & 0 & 1 & 0 \\
0 & 0 & 0 & 1
\end{array}\right]\left[\begin{array}{l}
0.3 \\
0.3 \\
0.3 \\
0.3
\end{array}\right]=\left[\begin{array}{l}
0.3 \\
0.3 \\
0.3 \\
0.3
\end{array}\right]
$$

\subsubsection{Cálculo da Matriz $P_{k}^{(-)}$}

O cálculo de $P_{k}^{(-)}$que indica a propagação da matriz de covariância do erro de estimação baseia-se na equação 4.37 que depende de

$P_{o}^{(+)}$: condição inicial da matriz de covariância do erro de estimação, matriz conhecida 4.42;

$\Phi_{k-1}^{t}$ : transposta da matriz de transição de estado, matriz identidade 4.43 de ordem $\left(n e_{x} n e\right)$;

$Q_{k-1}$ : matriz de covariância do ruído no estado, com $Q_{k}=\rho_{w}^{2} I_{n e}$, onde $\rho_{w}^{2}$ é a variância do ruído de estado. Por simplicidade, considera-se $Q_{k-1}$ uma matriz diagonal com elementos iguais, de dimensão $\left(n e_{x} n e\right)$. A matriz $Q_{k-1}$ é definida da seguinte forma por experimentação numérica

$$
Q_{k-1}=\left[\begin{array}{rrrr}
5 * 10^{-5} & 0 & 0 & 0 \\
0 & 5 * 10^{-5} & 0 & 0 \\
0 & 0 & 5 * 10^{-5} & 0 \\
0 & 0 & 0 & 5 * 10^{-5}
\end{array}\right]
$$

Em forma geral, a dimensão da matriz $P_{k}^{(-)}$é obtida explicitando as dimensões dos demais componentes da expressão 4.37 : 


$$
P_{k\left(n e_{x} n e\right)}^{(-)}=\Phi_{k-1\left(n e_{x} n e\right)} P_{k-1\left(n e_{x} n e\right)}^{(+)} \Phi_{k-1\left(n e_{x} n e\right)}^{t}+Q_{k-1\left(n e_{x} n e\right)}
$$

Logo, para o exemplo de 4 elementos

$$
P_{k}^{(-)}=\left[\begin{array}{rrrr}
0.05005 & 0 & 0 & 0 \\
0 & 0.05005 & 0 & 0 \\
0 & 0 & 0.05005 & 0 \\
0 & 0 & 0 & 0.05005
\end{array}\right]
$$

\subsubsection{Cálculo do Ganho de Kalman $G_{k}$}

O cálculo do ganho de Kalman $G_{k}$ baseia-se na equação 4.38, aqui repetida para facilitar a leitura,

$$
G_{k}=P_{k}^{(-)} H_{k}^{(n f) t}\left(\hat{\sigma}_{k}^{(-)}\right)\left[H_{k}^{(n f)}\left(\hat{\sigma}_{k}^{(-)}\right) P_{k}^{(-)} H_{k}^{(n f) t}\left(\hat{\sigma}_{k}^{(-)}\right)+R_{k}\right]^{-1}
$$

onde

$P_{k}^{(-)}$: propagação da matriz de covariância de erro de estimação, calculada em 4.46;

$H_{k}^{(n f)}\left(\hat{\sigma}_{k}^{(-)}\right)$: matriz de sensibilidade;

$H_{k}^{(n f) t}\left(\hat{\sigma}_{k}^{(-)}\right)$: matriz de sensibilidade transposta;

$R_{k}$ : matriz de covariância do ruído nas medidas $R_{k}=\rho_{v}^{2} I_{n f}$, onde $\rho_{v}^{2}$ : variância do ruído na medida.

A matriz de sensibilidade é obtida pela equação 4.25 , em função do vetor de condutividades $\hat{\sigma}_{k}^{(-)}$. O número de eletrodos é denotado $n f$, Tomam-se apenas as $n f$ primeiras linhas da matriz de sensibilidade, que são relacionadas aos nós que representam eletrodos, para formar $H_{k}^{(n f)}$ a partir de $H_{k}$.

No exemplo de 4 elementos $H_{k}^{(n f)}$ torna-se

$$
H_{k}^{(n f)}\left(\hat{\sigma}_{k}^{(-)}\right)=-Y^{-1} \frac{\partial Y}{\partial \sigma_{i}} Y^{-1} c_{1}=\left[\begin{array}{rrrr}
0 & 0 & 0 & 0 \\
-1.851 & -9.259 * 10^{-1} & 0 & -9.259 * 10^{-1} \\
-9.259 * 10^{-1} & -9.259 * 10^{-1} & 0 & 0 \\
-9.259 * 10^{-1} & -9.259 * 10^{-1} & 0 & 0
\end{array}\right]
$$

Por hipótese $R_{k}$ é uma matriz diagonal de dimensão $\left(n f_{x} n f\right)$ com elementos iguais. No exemplo de 4 elemento $R_{k}$ torna-se

$$
R_{k}=\left[\begin{array}{cccc}
8 & 0 & 0 & 0 \\
0 & 8 & 0 & 0 \\
0 & 0 & 8 & 0 \\
0 & 0 & 0 & 8
\end{array}\right]
$$


onde o valor do elemento na diagonal foi definido por experimentação numérica. Esta matriz contém a variância do ruído na medida de voltagem em cada eletrodo e depende da eletrônica do sistema.

No exemplo de 4 elementos resulta,

$$
G_{k}=\left[\begin{array}{rrrr}
0 & -0.01108 & -0.005497 & -0.005497 \\
0 & -0.005438 & -0.005585 & -0.005585 \\
0 & 0 & 0 & 0 \\
0 & -0.005615 & 0.000088 & 0.000088
\end{array}\right]
$$

\subsubsection{Cálculo da Matriz $P_{k}^{(+)}$}

O cálculo de $P_{k}^{(+)}$que é a atualização da matriz de covariância do erro de estimação baseia-se na equação 4.39 , aqui repetida para facilitar a leitura,

$$
P_{k}^{(+)}=\left[I-G_{k} H_{k}^{(n f)}\left(\hat{\sigma}_{k}^{(-)}\right)\right] P_{k}^{(-)}
$$

onde

$I$ : matriz de identidade de dimensão $\left(n e_{x} n e\right), \operatorname{com} n e$ sendo o número de elementos finitos da malha;

$G_{k}:$ ganho de Kalman, calculado em 4.50;

$H_{k}^{(n f)}\left(\hat{\sigma}_{k}^{(-)}\right)$: matriz de sensibilidade, calculada em 4.48;

$P_{k}^{(-)}$: propagação da matriz de covariância de erro de estimação, calculada em 4.46.

Em forma geral, a dimensão de $P_{k}^{(+)}$é obtida explicitando as dimensões dos demais componentes da expressão:

$$
P_{k\left(n e_{x} n e\right)}^{(+)}=\left[I_{\left(n e_{x} n e\right)}-G_{k\left(n e_{x} n f\right)} H_{k\left(n f_{x} n e\right)}^{(n f)}\left(\hat{\sigma}_{k}^{(-)}\right)\right] P_{k\left(n e_{x} n e\right)}^{(-)}
$$

Logo, para o exemplo de 4 elementos resulta,

$$
P_{k}^{(+)}=\left[\begin{array}{rrrr}
0.048517 & -0.001021 & 0 & -0.000512 \\
-0.001021 & 0.049280 & 0 & -0.000252 \\
0 & 0 & 0.05005 & 0 \\
-0.000512 & -0.000252 & 0 & 0.049790
\end{array}\right]
$$

\subsubsection{Cálculo do Vetor $\hat{\sigma}_{k}^{(+)}$}

O cálculo do vetor $\hat{\sigma}_{k}^{(+)}$que é a atualização dos estados das estimativas baseia-se na equação 4.40, aqui reproduzida para facilitar a leitura, 


$$
\hat{\sigma}_{k}^{(+)}=\hat{\sigma}_{k}^{(-)}+G_{k}\left[v_{k}^{(n f)}-h_{k}^{(n f)}\left(\hat{\sigma}_{k}^{(-)}\right)\right]
$$

onde

$\hat{\sigma}_{k}^{(-)}$: propagação da estimativa do estado, vetor calculado em 4.44;

$G_{k}:$ ganho de Kalman, calculado em 4.50;

$v_{k}^{(n f)}$ : novo vetor de medidas;

$h_{k}^{(n f)}\left(\hat{\sigma}_{k}^{(-)}\right)$: vetor de voltagens calculado para os $n f$ eletrodos.

No caso de dados experimentais $v_{k}^{(n f)}$, é o vetor de medidas das voltagens nos eletrodos $n f$, ou com dados simulados numericamente é o vetor de voltagens calculado aplicando a metodologia para a solução do problema direto, equação 4.16, com o padrão de corrente $C_{j}$ em função das condutividades esperadas. Neste exemplo espera-se obter uma estimativa da distribuição de condutividades de $0.1(\Omega m)^{-1}$ para todos os 4 elementos. Neste caso obtém-se :

$$
v_{k}^{(n f)}=\left[\begin{array}{r}
0 \\
3.70370 \\
1.85185 \\
1.85185
\end{array}\right]+w_{k}
$$

onde $w_{k}$ é ruído branco Gaussiano de média nula, no exemplo de 4 elementos considera-se o ruído nulo.

O vetor de voltagens $h_{k}^{(n f)}\left(\hat{\sigma}_{k}^{(-)}\right)$é obtido pelo produto da inversa da matriz de condutividade pelo padrão de corrente $C_{j}$, conforme a equação 4.16. No caso de estudo, a matriz de condutividade é determinada substituindo a distribuição de condutividades estimada da equação 4.44 na expressão 4.24. Logo, o vetor $h_{k}^{(n f)}\left(\hat{\sigma}_{k}^{(-)}\right)$é dado por :

$$
h_{k}^{(n f)}\left(\hat{\sigma}_{k}^{(-)}\right)=\left[\begin{array}{r}
0 \\
1.23457 \\
0.61728 \\
0.61728
\end{array}\right] .
$$

No exemplo de 4 elementos o estado atualizado torna-se

$$
\hat{\sigma}_{k}^{(+)}=\left[\begin{array}{c}
0.255097 \\
0.270098 \\
0.300000 \\
0.285000
\end{array}\right]
$$

O vetor $\hat{\sigma}_{k}^{(+)}$representa a nova estimativa da distribuição de condutividades dos 4 elementos da malha de elementos finitos na primeira iteração. 


\subsubsection{Critério de Convergência}

O resíduo de medida $r_{v}$ dada pela equação 4.57 é utilizado para monitorar a convergência do Filtro, segundo Fleury [13, 14], e é definido como :

$$
r_{v}=\left\{1 / n f \sum_{j=1}^{n f}\left[v_{k}^{(n f)}(j)-h_{k}^{(n f)}(j)\right]\right\} / \rho_{v}, j=1,2, \ldots, n f
$$

onde

$r_{v}:$ resíduo normalizado;

$n f:$ número de eletrodos;

$v_{k}^{(n f)}$ : medida real, dados experimentais das voltagens nos eletrodos;

$h_{k}^{(n f)}$ : valor calculado das voltagens nos eletrodos;

$\rho_{v}$ : desvio padrão dos ruídos na medida.

O processo é considerado convergente desde que $-3 \rho_{v} \leq r_{v} \leq 3 \rho_{v}$ e que $E\left[r_{v}\right]=0$.

\subsubsection{Simulações}

Para finalizar, aplicando toda a metodologia exposta neste capítulo para a solução do problema inverso utilizando o Filtro de Kalman no exemplo da Figura 4.1, realizaram-se três simulações para estudar o comportamento do algoritmo desenvolvido em duas formas de solução.

\section{Simulação 1}

Na Tabela 4.1 mostra-se o resumo dos parâmetros utilizados para a metodologia implementada na solução do problema inverso aplicando o Filtro de Kalman e dos resultados obtidos $\hat{\sigma}_{k}^{(+)}$após 90 iterações. Neste exemplo espera-se obter uma distribuição de condutividades homogêneas $\sigma$, sendo que foi necessário gerar valores de voltagens através da formulação do problema direto considerando uma distribuição de condutividades homogênea de $0.1(\Omega m)^{-1}$ para os 4 elementos. Neste caso, não foi adicionado ruído ao vetor $v_{k}^{(n f)}$ de dados numéricos das voltagens nos eletrodos.

onde

$\hat{\sigma}_{o}^{(+)}$: condição inicial do vetor de condutividades;

$\sigma:$ valor de condutividade esperado;

$\hat{\sigma}_{90}^{(+)}$distribuição de condutividades estimado.

A distribuição de condutividades é obtida depois de 90 iterações. Este número de iterações é selecionado somente como critério de comparação entre os três tipos de simulações estudados 
Tabela 4.1: Simulação 1. Parâmetros para o Filtro de Kalman aplicado ao exemplo da Figura 4.1, sem adicionar ruído ao vetor $v_{k}^{(n f)}$. Distribuição de condutividades homogênea esperada de $0.1(\Omega m)^{-1}$

\begin{tabular}{|l||c|c|c|}
\hline \hline Elemento No. & $\hat{\sigma}_{o}^{(+)}$ & $\sigma$ & $\hat{\sigma}_{90}^{(+)}$ \\
\hline 1 & 0.3 & 0.1 & 0.098787 \\
\hline 2 & 0.3 & 0.1 & 0.098911 \\
\hline 3 & 0.3 & 0.1 & 0.104815 \\
\hline 4 & 0.3 & 0.1 & 0.104815 \\
\hline \hline 1́ndice de erro & \multicolumn{3}{|c|}{0.000047} \\
\hline \hline$P_{o}^{(+)}$ & \multicolumn{3}{|c|}{$5 * 10^{-2} I_{4}$} \\
\hline$Q$ & \multicolumn{3}{|c|}{$5 * 10^{-5} I_{4}$} \\
\hline$R$ & \multicolumn{3}{|c|}{$8 I_{4}$} \\
\hline \hline
\end{tabular}

aplicando a metodologia do Filtro de Kalman para a solução do problema de TIE.

O índice de erro é calculado segundo

$$
\sum_{i=1}^{n e}\left(\sigma-\hat{\sigma}_{k}^{(+)}\right)^{2}
$$

com ne igual ao número de elementos.

Os valores nos componentes das matrizes $P_{o}^{(+)}, Q$ e $R$ utilizados neste exemplo foram obtidos por experimentação numérica.

Na Figura 4.7 mostra-se a evolução temporal das condutividades dos 4 elementos para a simulação 1, após 90 iterações. As estimativas foram obtidas a partir do algoritmo desenvolvido com o software Octave.

Na Figura 4.8 apresenta-se o resíduo normalizado $r_{v}$, onde a convergência é verificada visto que $E\left[r_{v}\right]=0$ e que $-3 \rho_{v} \leq r_{v} \leq 3 \rho_{v}$, onde $\rho_{v}=2.83$. 


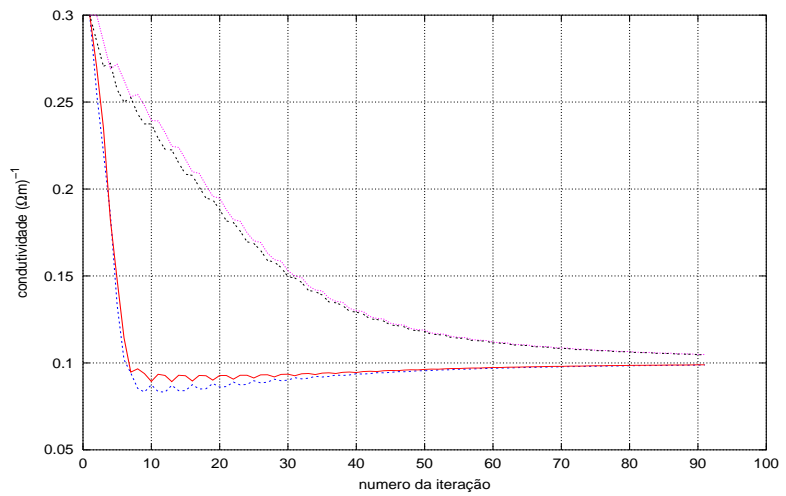

Figura 4.7: Simulação 1. Variação das condutividades dos 4 elementos, segundo os parâmetros da Tabela 4.1, sem adicionar ruído ao vetor $v_{k}^{(n f)}$ após 90 iterações. Distribuição de condutividade homogênea esperada de $0.1(\Omega m)^{-1}$.

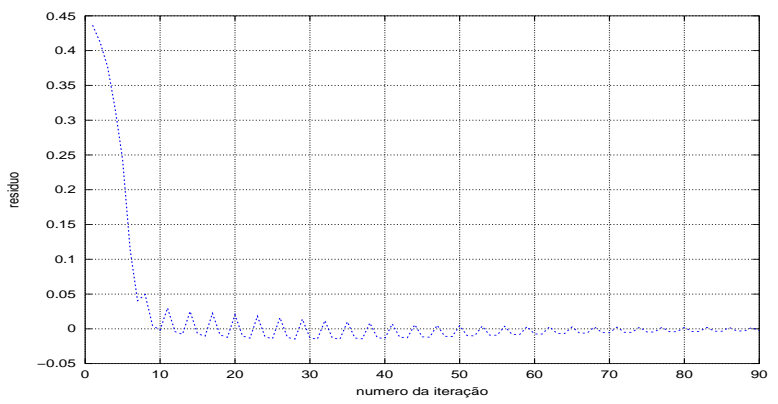

Figura 4.8: Resíduo normalizado, $r_{v}$, da simulação 1. Verifica-se $-8.49 \leq r_{v} \leq 8.49$. 


\section{Simulação 2}

A Tabela 4.2 contém os parâmetros utilizados na segunda simulação. Adicionou-se ruído branco Gaussiano de média nula ao vetor $v_{k}^{(n f)}$ com desvio padrão $5 \%$ da máxima voltagem calculada por meio do problema direto. Os valores de voltagens foram gerados através da formulação do problema direto considerando condutividade de $0.1(\Omega m)^{-1}$ nos 4 elementos.

Neste exemplo, os valores da matriz $P_{o}^{(+)}$são 10 vezes menores. A variância do ruído no estado, na matriz $Q$ aumentou-se 1000 vezes e a matriz $R$ permanece inalterada. Os valores nos componentes das matrizes $P_{o}^{(+)}, Q$ e $R$ utilizados neste segundo exemplo foram obtidos por experimentação numérica.

Tabela 4.2: Simulação 2. Parâmetros para o Filtro de Kalman aplicado ao exemplo da Figura 4.1, adicionando ruído branco Gaussiano de média nula ao vetor $v_{k}^{(n f)}$ com desvio padrão $5 \%$ da máxima voltagem calculada por meio do problema direto. Distribuição de condutividades homogênea esperada de $0.1(\Omega m)^{-1}$

\begin{tabular}{|l||c|c|c|}
\hline \hline Elemento No. & $\hat{\sigma}_{o}^{(+)}$ & $\sigma$ & $\hat{\sigma}_{90}^{(+)}$ \\
\hline 1 & 0.3 & 0.1 & 0.093776 \\
\hline 2 & 0.3 & 0.1 & 0.098473 \\
\hline 3 & 0.3 & 0.1 & 0.103785 \\
\hline 4 & 0.3 & 0.1 & 0.099087 \\
\hline \hline 1́ndice de erro & \multicolumn{3}{|c|}{0.000056} \\
\hline \hline$P_{o}^{(+)}$ & \multicolumn{3}{|c|}{$5 * 10^{-3} I_{4}$} \\
\hline$Q$ & \multicolumn{3}{|c|}{$5 * 10^{-2} I_{4}$} \\
\hline$R$ & \multicolumn{3}{|c|}{$8 I_{4}$} \\
\hline \hline
\end{tabular}

A Figura 4.9 contém a evolução temporal das estimativas de condutividades dos 4 elementos após 90 iterações, usando os parâmetros da Tabela 4.2. Observa-se que as estimativas de condutividades alcançam o valor esperado já desde a iteração 20, entretanto o Filtro oscila em torno do estado esperado.

A Figura 4.10 apresenta o resíduo normalizado $r_{v}$, onde a condição de convergência é verificada visto que $E\left[r_{v}\right]=0$ e que $-3 \rho_{v} \leq r_{v} \leq 3 \rho_{v}$, onde $\rho_{v}=2.83$.

\section{Simulação 3}

A Tabela 4.3 contém os parâmetros utilizados na terceira simulação aplicando a metodologia do Filtro de Kalman, adicionando ruído branco Gaussiano de média nula ao vetor $v_{k}^{(n f)}$ com desvio 


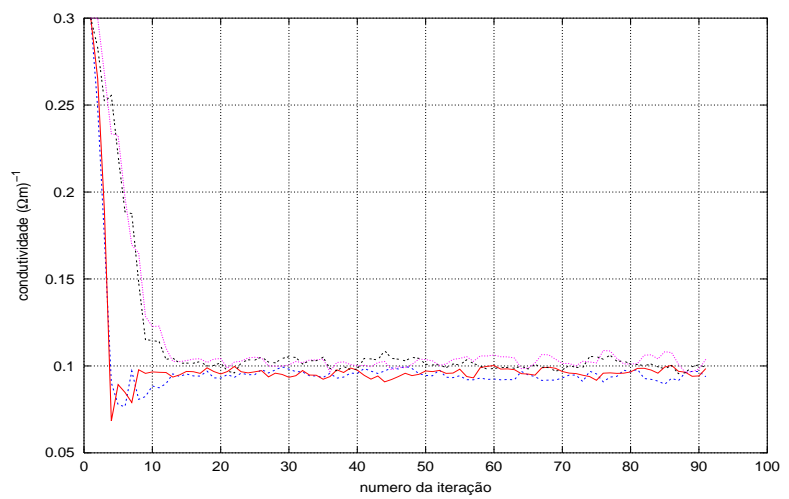

Figura 4.9: Simulação 2. Variação das condutividades dos 4 elementos segundo parâmetros da Tabela 4.2, após 90 iterações, adicionando ruído branco Gaussiano de média nula ao vetor $v_{k}^{(n f)}$ com desvio padrão $5 \%$ da máxima voltagem calculada por meio do problema direto. Distribuição de condutividade homogênea esperada de $0.1(\Omega m)^{-1}$.

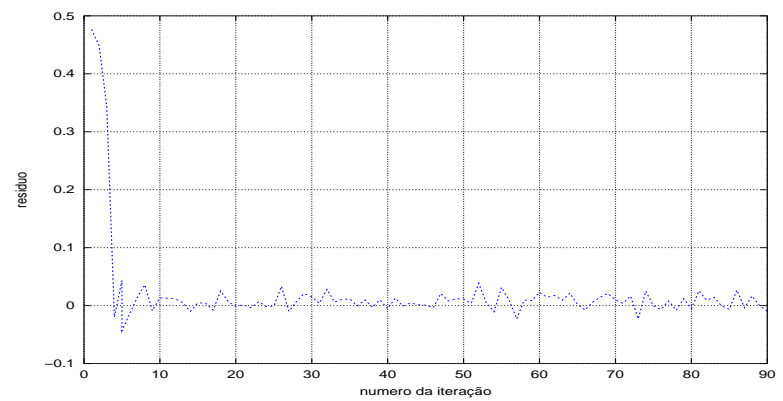

Figura 4.10: Resíduo normalizado, $r_{v}$, da simulação 2. Verifica-se $-8.49 \leq r_{v} \leq 8.49$. 
padrão $7 \%$ da máxima voltagem calculada por meio do problema direto. Neste exemplo espera-se obter uma distribuição de condutividades não homogêneas, sendo que foi necessário gerar valores de voltagens através da formulação do problema direto considerando uma distribuição de condutividades não homogênea, segundo vetor o $\hat{\sigma}_{o}^{(+)}$na Tabela 4.3.

Tabela 4.3: Simulação 3. Parâmetros para o Filtro de Kalman aplicado ao exemplo da Figura 4.1, adicionando ruído branco Gaussiano de média nula ao vetor $v_{k}^{(n f)}$ com desvio padrão $7 \%$ da máxima voltagem calculada por meio do problema direto. Distribuição de condutividades esperada, não homogênea

\begin{tabular}{|l||c|c|c|}
\hline \hline Elemento No . & $\hat{\sigma}_{o}^{(+)}$ & $\sigma$ & $\hat{\sigma}_{90}^{(+)}$ \\
\hline 1 & 0.35 & 0.20 & 0.17344 \\
\hline 2 & 0.30 & 0.10 & 0.11280 \\
\hline 3 & 0.25 & 0.35 & 0.34847 \\
\hline 4 & 0.20 & 0.30 & 0.30910 \\
\hline \hline índice de erro & \multicolumn{3}{|c|}{0.00095} \\
\hline \hline$P_{o}^{(+)}$ & \multicolumn{3}{|c|}{$5 * 10^{-3} I_{4}$} \\
\hline$Q$ & \multicolumn{3}{|c|}{$3 * 10^{-1} I_{4}$} \\
\hline$R$ & \multicolumn{3}{|c|}{$4 I_{4}$} \\
\hline \hline
\end{tabular}

Os valores das componentes das matrizes $P_{o}^{(+)}, Q$ e $R$ utilizados neste exemplo são obtidos por experimentação numérica.

A Figura 4.11 contém a evolução temporal das estimativas de condutividades dos 4 elementos após 90 iterações segundo os parâmetros da Tabela 4.3.

Na Figura 4.12 apresenta-se o resíduo normalizado $r_{v}$, onde a convergência é verificada visto que $E\left[r_{v}\right]=0$ e $-3 \rho_{v} \leq r_{v} \leq 3 \rho_{v}$, onde $\rho_{v}=2$.

\subsubsection{Simulações com novos parâmetros $P_{o}^{(+)}, Q$ e $R$}

Apresenta-se aqui uma forma alternativa para a solução do problema inverso, utilizando o Filtro de Kalman no exemplo da Figura 4.1, a partir de novos valores para as componentes das matrizes $P_{o}^{(+)}, Q$ e $R$, segundo Brogan [6]. Para o qual considera-se o seguinte :

- $R$ : matriz de covariância do ruído nas medidas;

- $P_{o}$ : condição inicial da matriz de covariância do erro de estimação;

Considerase a hipótese que $R$ e $P_{o}$ são matrizes diagonais, com elementos iguais.

- $P_{o}^{(+)}$: estimativa inicial da matriz de covariância do erro de estimação, assim : 


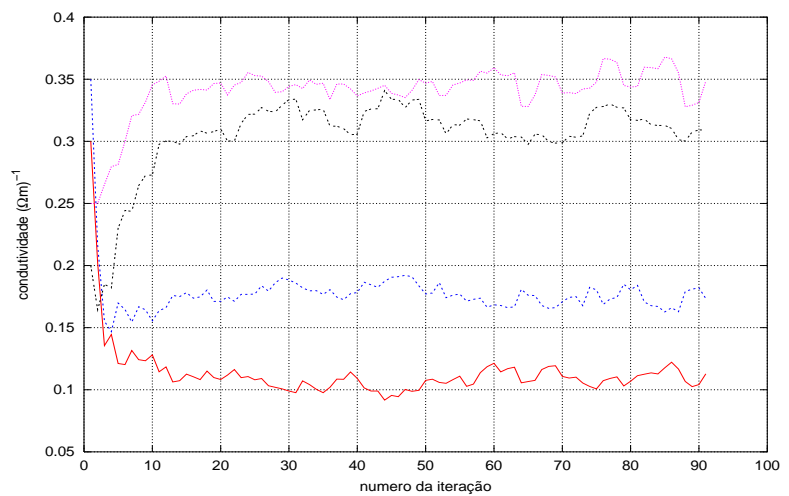

Figura 4.11: Simulação 3. Variação das condutividades dos 4 elementos segundo parâmetros da Tabela 4.3, após 90 iterações, adicionando ruído branco Gaussiano de média nula ao vetor $v_{k}^{(n f)}$ com desvio padrão $7 \%$ da máxima voltagem calculada por meio do problema direto. Distribuição de condutividade esperada, não homogênea.

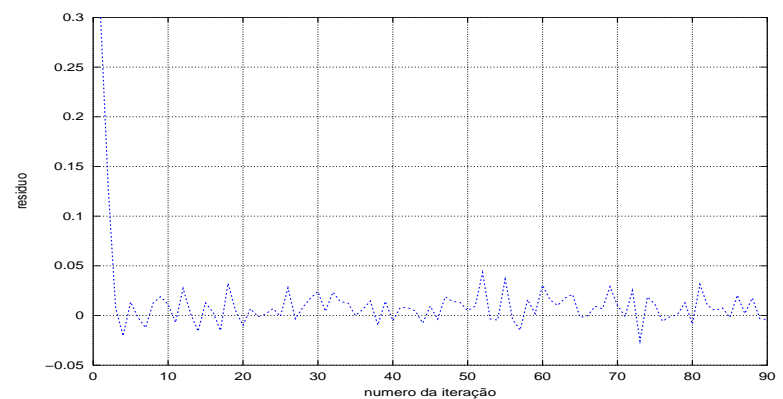

Figura 4.12: Resíduo normalizado, $r_{v}$, da simulação 3. Verifica-se $-6 \leq r_{v} \leq 6$. 


$$
P_{o}^{(+)}=\left[P_{o}^{-1}+H^{t} R^{-1} H\right]^{-1}
$$

onde

$H$ é a matriz de sensibilidade, calculada em 4.48, com os valores da condição inicial do vetor de condutividades $\hat{\sigma}_{o}^{(+)}$;

- $Q$ é a matriz de covariância do ruído no estado, a partir da equação 4.59 .

$$
Q=0.001 * P_{o}^{(+)}
$$

Utilizando a equação 4.59 simulam-se novamente os três casos anteriores.

\section{Simulação 1, com novos parâmetros}

A Tabela 4.4 contém os parâmetros utilizados pelo Filtro de Kalman, utilizando a equação 4.59.

Tabela 4.4: Simulação 1. Novos parâmetros do Filtro de Kalman aplicado ao exemplo da Figura 4.1, sem adicionar ruído ao vetor $v_{k}^{(n f)}$. Distribuição de condutividades homogênea esperada de $0.1(\Omega m)^{-1}$

\begin{tabular}{|l||c|c|c|}
\hline \hline Elemento No. & $\hat{\sigma}_{o}^{(+)}$ & $\sigma$ & $\hat{\sigma}_{90}^{(+)}$ \\
\hline 1 & 0.3 & 0.1 & 0.099988 \\
\hline 2 & 0.3 & 0.1 & 0.099990 \\
\hline 3 & 0.3 & 0.1 & 0.100046 \\
\hline 4 & 0.3 & 0.1 & 0.100045 \\
\hline \hline índice de erro & \multicolumn{3}{||}{0.00000000437} \\
\hline \hline$R$ & \multicolumn{3}{|c|}{$0.08 I_{4}$} \\
\hline$P_{o}$ & \multicolumn{3}{|c|}{$5 * 10^{-3} I_{4}$} \\
\hline \hline
\end{tabular}

A Figura 4.13 mostra a evolução temporal das condutividades dos 4 elementos após 90 iterações. $\mathrm{O}$ algoritmo foi desenvolvido com o software Octave.

\section{Simulação 2, com novos parâmetros}

A Tabela 4.5 contém os parâmetros utilizados pelo Filtro de Kalman. Neste exemplo espera-se também obter una distribuição de condutividades homogêneas.

A Figura 4.14 mostra a evolução temporal das estimativas de condutividades dos 4 elementos após 90 iterações, segundo os parâmetros da Tabela 4.5 . 


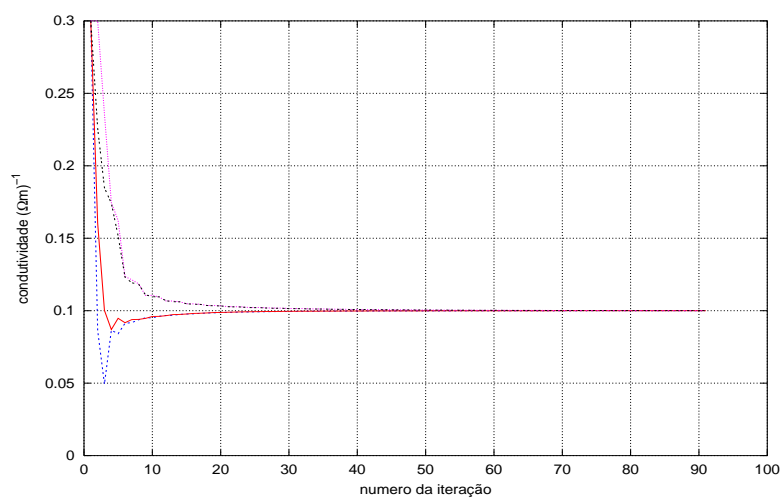

Figura 4.13: Simulação 1 com novos parâmetros. Variação das condutividades dos 4 elementos, segundo parâmetros da Tabela 4.4, sem adicionar ruído ao vetor $v_{k}^{(n f)}$, após 90 iterações. Distribuição de condutividade homogênea esperada de $0.1(\Omega m)^{-1}$.

Tabela 4.5: Simulação 2. Novos parâmetros do Filtro de Kalman aplicado ao exemplo da Figura 4.1, adicionando ruído branco Gaussiano de média nula ao vetor $v_{k}^{(n f)}$ com desvio padrão $5 \%$ da máxima voltagem calculada por meio do problema direto. Distribuição de condutividade homogênea esperada de $0.1(\Omega m)^{-1}$

\begin{tabular}{|l||c|c|c|}
\hline \hline Elemento No. & $\hat{\sigma}_{o}^{(+)}$ & $\sigma$ & $\hat{\sigma}_{90}^{(+)}$ \\
\hline 1 & 0.3 & 0.1 & 0.094331 \\
\hline 2 & 0.3 & 0.1 & 0.096888 \\
\hline 3 & 0.3 & 0.1 & 0.102557 \\
\hline 4 & 0.3 & 0.1 & 0.100000 \\
\hline \hline índice de erro & \multicolumn{3}{|c|}{0.000048} \\
\hline \hline$R$ & \multicolumn{3}{|c|}{$0.08 I_{4}$} \\
\hline$P_{o}$ & \multicolumn{3}{|c|}{$5 * 10^{-3} I_{4}$} \\
\hline \hline
\end{tabular}




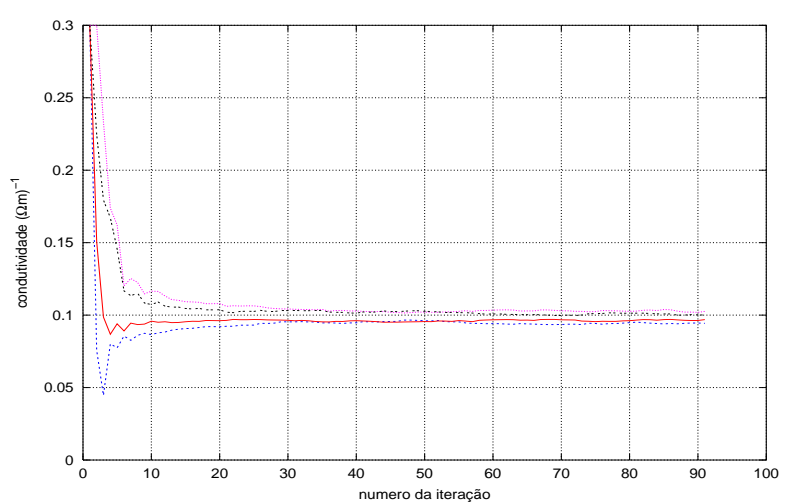

Figura 4.14: Simulação 2 com novos parâmetros. Variação das condutividades dos 4 elementos segundo parâmetros da Tabela 4.5. Após 90 iterações, adicionando ruído branco Gaussiano de média nula ao vetor $v_{k}^{(n f)}$ com desvio padrão $5 \%$ da máxima voltagem calculada por meio do problema direto. Distribuição de condutividade homogênea esperada de $0.1(\Omega m)^{-1}$.

\section{Simulação 3, com novos parâmetros}

A Tabela 4.6 contém os parâmetros utilizados aplicando a metodologia do Filtro de Kalman. Neste exemplo espera-se obter una distribuição de condutividades não homogêneas.

Os valores nos componentes das matrizes $P_{o}$ e $R$ utilizados neste exemplo são obtidos por experimentação numérica.

A Figura 4.15 mostra a evolução temporal das estimativas de condutividades dos 4 elementos após 90 iterações segundo os parâmetros da Tabela 4.6.

Com o objetivo de facilitar a comparação dos resultados obtidos, na Figura 4.16 são reproduzidos os gráficos das variações das condutividades dos 4 elementos para as seis simulações apresentadas anteriormente.

Como as simulações apresentadas anteriormente são númericas, os valores esperados de distribuição de condutividade são conhecidas, sendo para a primeira e segunda simulação uma distribuição de condutividades homogênea com valores de $0.1(\Omega m)^{-1}$ e para a terceira simulação uma distribuição de condutividades não homogênea. Nas simulações os valores de condutividades esperados são alcançados. 
Tabela 4.6: Simulação 3. Novos parâmetros do Filtro de Kalman aplicado ao exemplo da Figura 4.1, adicionando ruído branco Gaussiano de média nula ao vetor $v_{k}^{(n f)}$ com desvio padrão $7 \%$ da máxima voltagem calculada por meio do problema direto. Distribuição de condutividade esperada, não homogênea

\begin{tabular}{|l||c|c|c|}
\hline \hline Elemento No . & $\hat{\sigma}_{o}^{(+)}$ & $\sigma$ & $\hat{\sigma}_{90}^{(+)}$ \\
\hline 1 & 0.35 & 0.20 & 0.17403 \\
\hline 2 & 0.30 & 0.10 & 0.11042 \\
\hline 3 & 0.25 & 0.35 & 0.34925 \\
\hline 4 & 0.20 & 0.30 & 0.31296 \\
\hline \hline índice de erro & \multicolumn{3}{|c|}{0.00095} \\
\hline \hline$R$ & \multicolumn{3}{|c|}{$0.004 I_{4}$} \\
\hline$P_{o}$ & \multicolumn{3}{|c}{$9 * 10^{-2} I_{4}$} \\
\hline \hline
\end{tabular}

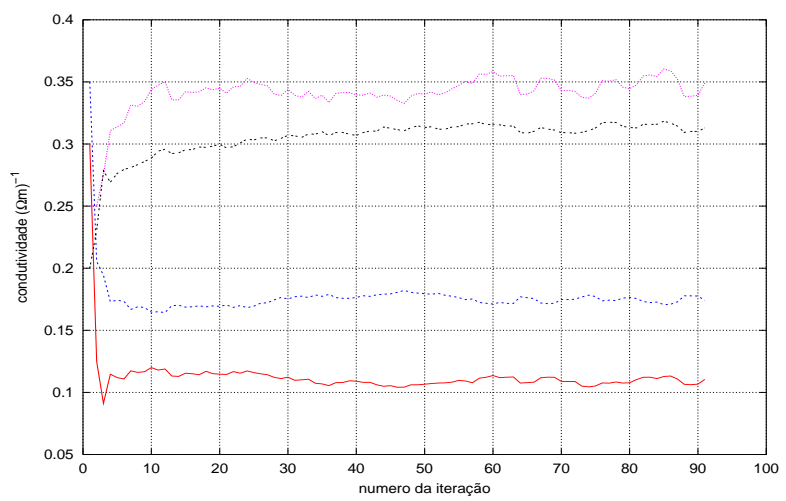

Figura 4.15: Simulação 3 com novos parâmetros. Variação das condutividades dos 4 elementos segundo parâmetros da Tabela 4.6. Após 90 iterações, adicionando ruído branco Gaussiano de média nula ao vetor $v_{k}^{(n f)}$ com desvio padrão $7 \%$ da máxima voltagem calculada por meio do problema direto. Distribuição de condutividade esperada, não homogênea. 


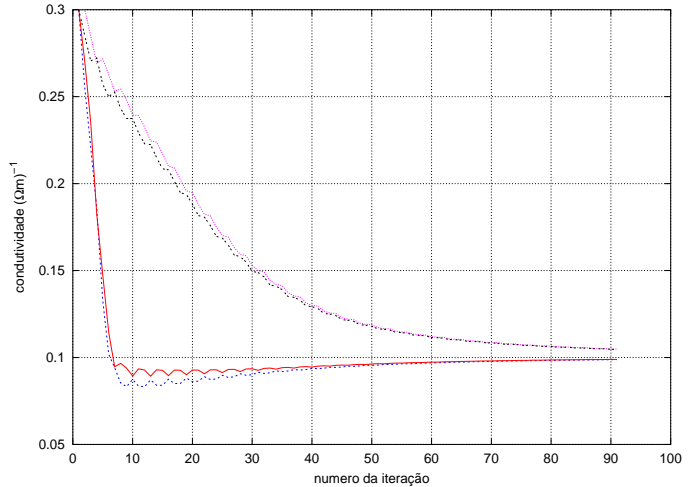

(a)

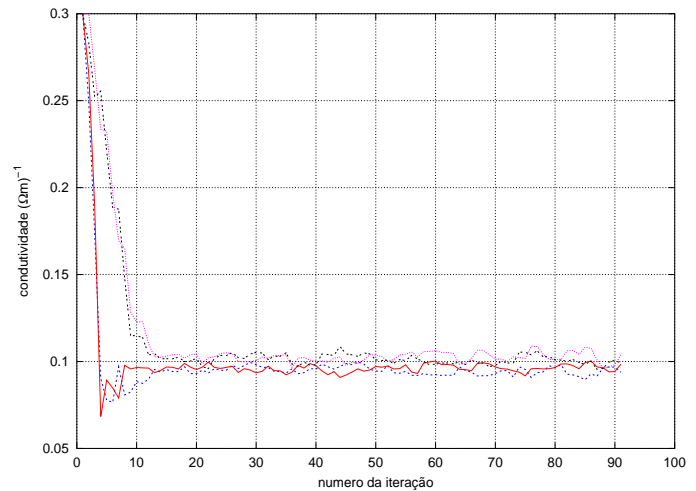

(c)

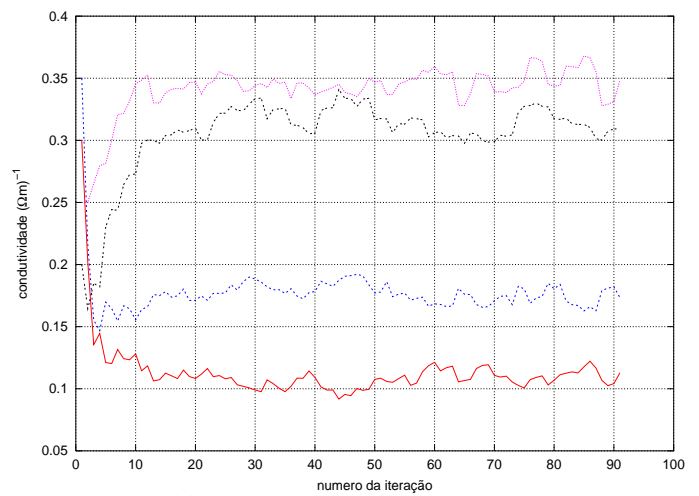

(e)

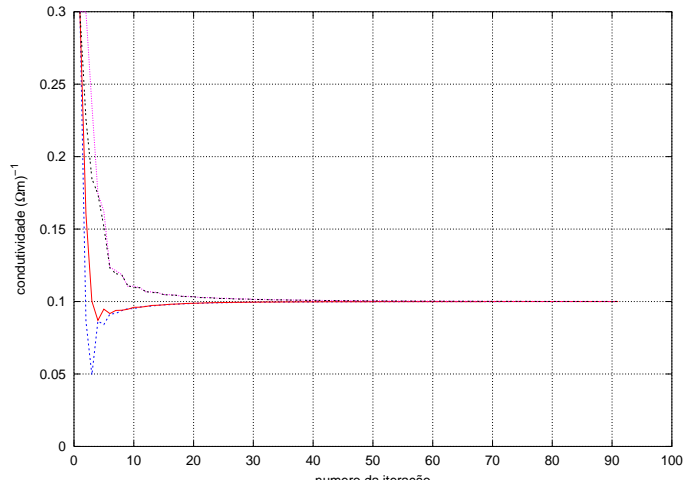

(b)

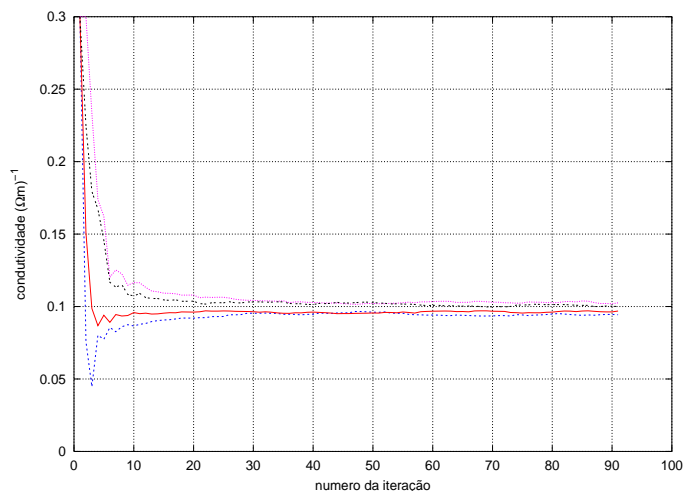

(d)

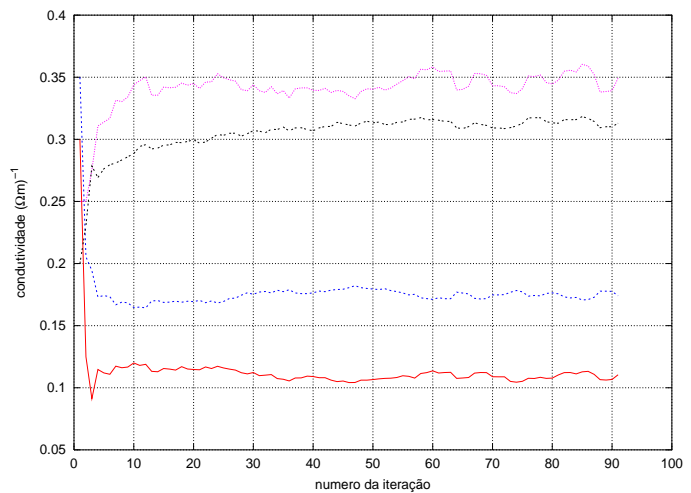

(f)

Figura 4.16: Comparação dos resultados das variações das condutividades dos 4 elementos. Parâmetros $P_{o}^{(+)}, Q$ e $R$ obtidos por experimentação numérica : (a) Simulação 1, (c) Simulação 2, (e) Simulação 3. E com novos parâmetros $P_{o}^{(+)}$e $Q$ obtidos a partir das considerações de Brogan : (b) Simulação 1, (d) Simulação 2, (f) Simulação 3. 
O uso das equações 4.58 e 4.59 permite ajustar os parâmetros $P_{o}^{(+)}$e $Q$ que levam a um melhor desempenho do Filtro Estendido de Kalman.

Neste trabalho é empregada esta metodologia para estimar a distribuição de condutividades num phantom com o objetivo de identificar a posição e valor da condutividade de um objeto dentro do phantom. 


\section{Capítulo 5}

\section{RENUMERAÇÃO DA MALHA E MATRIZ DE CONDUTIVIDADE DIAGONAL DE BANDA}

Na solução do problema de TIE, a matriz de condutividade e a sua inversa são importantes nas estimativas da distribuição de condutividades. O objetivo deste capítulo é determinar um método para converter a matriz de condutividade, que é uma matriz esparsa, em matriz de banda limitada, a fim de diminuir o tempo computacional para o cálculo de sua inversa e minimizar os erros numéricos, ou seja, diminuir a propagação de erro. Dois conceitos que levam ao resultado esperado são : a renumeração da malha de elementos finitos e a matriz de condutividade diagonal de banda.

\subsection{Renumeração da Malha}

Foi mencionado no capítulo anterior o método para facilitar a discretização do domínio que representa a seção do tórax, em ne elementos triangulares e com $n n$ nós na malha. Utiliza-se o módulo de geração automática de malhas Corduroy que faz parte do software FElt e a partir do qual obtêmse uma topologia representada nos arquivos, cd.dat e nodes.dat, que são usados no cálculo da matriz de condutividade. Este software também dispõe de um módulo de renumeração da malha de elementos finitos que permite obter uma nova topologia representada nos arquivos, $c d b$.dat e nodesb.dat. Com esta nova topologia obtém-se uma nova matriz de condutividade $Y$. 


\subsection{Matriz de Condutividade Diagonal de Banda}

Após a renumeração dos nós da malha de elementos finitos, a nova matriz de condutividade assume a forma denominada diagonal de banda.

O conceito de matriz diagonal de banda é clássico e pode-se encontrar facilmente a sua implementação [29]. Gibbs et al., no artigo An Algorithm for Reducing the Bandwidth and Profile of a Sparse Matrix [16], apresentam a metodologia para implementar a redução de matrizes esparsas em matrizes de banda, na solução de equações algébricas lineares.

A matriz diagonal de banda é guardada e manipulada na chamada forma compacta, que é a matriz constituída pelos elementos das diagonais da banda da matriz. Com rotinas que manipulam esta forma compacta da matriz de condutividade calcula-se sua inversa.

A solução do sistema na forma compacta é obtida mais rapidamente que na forma esparsa da matriz de condutividade $\left(n n_{x} n n\right)$. A outra vantagem é que se requer menos espaço de armazenamento nos cálculos de sua inversa, o qual leva a uma importante redução do custo computacional. Diminuem o número de operações e os erros numéricos.

Foram empregados algoritmos do Numerical Recipes [29] para o cálculo da inversa da matriz de condutividade na forma compacta.

Considere o exemplo da Figura 4.1 (aqui representado pela Figura 5.1(a)). Renumerando a malha de elementos finitos de forma tal que o número dos nós fique ordenado em sentido ascendente desde o nó 1, obtém-se o resultado ilustrado pela Figura 5.1(b).

Com a metodologia para a solução do problema direto exposta no capítulo anterior, calculouse a matriz de condutividade $Y$ para o caso (a) da Figura 5.1, a qual é uma matriz de condutividade apresentada na equação 5.1 .

$$
[Y]=\left[\begin{array}{rrrrr}
0.0189 & 0 & 0 & 0 & -0.0189 \\
0 & 0.0189 & 0 & 0 & -0.0189 \\
0 & 0 & 0.0189 & 0 & -0.0189 \\
0 & 0 & 0 & 0.0189 & -0.0189 \\
-0.0189 & -0.0189 & -0.0189 & -0.0189 & 0.0756
\end{array}\right]
$$

Utilizando a mesma metodologia para o cálculo da matriz de condutividade para a malha renumerada, caso (b) da Figura 5.1, agora com a nova matriz de coordenadas $(x, y)$ dos nós denominada $c d b . d a t$ e outra matriz com os dados dos nós de cada um dos elementos denominada nodesb.dat 


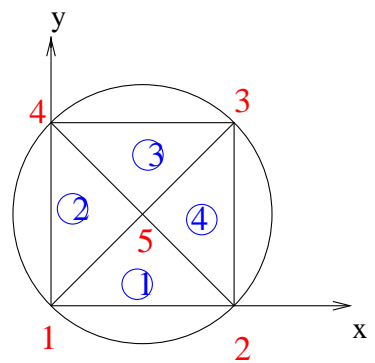

(a)

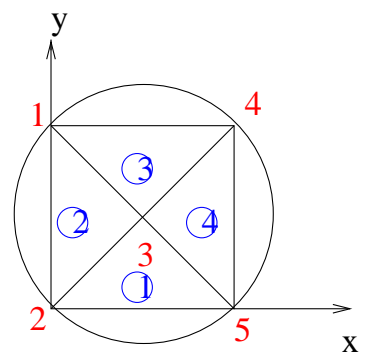

(b)

Figura 5.1: Malha de elementos finitos com 4 elementos e 5 nós, (a) malha inicial, (b) malha renumerada. 


$$
\begin{gathered}
\text { cdb.dat }=\left[\begin{array}{ll}
x_{1} & y_{1} \\
x_{2} & y_{2} \\
x_{3} & y_{3} \\
x_{4} & y_{4} \\
x_{5} & y_{5}
\end{array}\right]=\left[\begin{array}{ll}
0 & 0.30 \\
0 & 0 \\
0.15 & 0.15 \\
0.30 & 0.30 \\
0.30 & 0
\end{array}\right] \\
\text { nodesb.dat }=\left[\begin{array}{lll}
2 & 5 & 3 \\
2 & 3 & 1 \\
1 & 3 & 4 \\
5 & 4 & 3
\end{array}\right]
\end{gathered}
$$

obtém-se a matriz de condutividade em forma de diagonal de banda $Y b$ :

$$
[Y b]=\left[\begin{array}{rrrrr}
0.018900 & 0.000000 & -0.018900 & 0.000000 & 0.000000 \\
0.000000 & 0.018900 & -0.018900 & 0.000000 & 0.000000 \\
-0.018900 & -0.018900 & 0.075600 & -0.018900 & -0.018900 \\
0.000000 & 0.000000 & -0.018900 & 0.018900 & 0.000000 \\
0.000000 & 0.000000 & -0.018900 & 0.000000 & 0.018900
\end{array}\right]
$$

Observa-se que a nova matriz de condutividade $Y b$ obtida com a renumeração da malha inicial de elementos finitos é da forma diagonal de banda e simétrica, e a forma compacta $Y c$ da matriz de condutividade diagonal de banda é dada por :

$$
[Y c]=\left[\begin{array}{rrrrr}
x & x & 0.018900 & 0.000000 & -0.018900 \\
x & 0.000000 & 0.018900 & -0.018900 & 0.000000 \\
-0.018900 & -0.018900 & 0.075600 & -0.018900 & -0.018900 \\
0.000000 & -0.018900 & 0.018900 & 0.000000 & x \\
-0.018900 & 0.000000 & 0.018900 & x & x
\end{array}\right]
$$

\subsection{Cinco Casos de Estudo de Malhas Renumeradas}

Consideram-se cinco tipos de malhas de elementos planos triangulares, representando diferentes discretizações de uma seção transversal do tórax, e as respectivas malhas renumeradas,

- caso 1. Malha que possui $n e=4$ elementos e $n n=5$ nós, dos quais $n f=4$ são nós do contorno e correspondem aos eletrodos, caso já mencionado na seção anterior. Na Figura 5.1 se mostra em (a) a malha inicial e em (b) a malha renumerada;

- caso 2. Malha que possui $n e=32$ elementos e $n n=25$ nós, dos quais $n f=16$, como se mostra na Figura 5.2 (malha inicial) e na Figura 5.3 (malha renumerada); 
- caso 3. Malha que possui $n e=96$ elementos e $n n=65$ nós, dos quais $n f=16$, como se mostra na Figura 5.4 (malha inicial) e na Figura 5.5 (malha renumerada);

- caso 4. Malha que possui $n e=118$ elementos e $n n=92$ nós, dos quais $n f=16$, como se mostra na Figura 5.6 (malha inicial) e na Figura 5.7 (malha renumerada);

- caso 5. Malha que possui $n e=182$ elementos e $n n=156$ nós, dos quais $n f=32$ dos nós do contorno correspondem aos eletrodos, como se mostra na Figura 5.8 para a malha inicial e na Figura 5.9 para a malha renumerada.

A partir dos dados das matrizes $c$ d.dat e nodes.dat, gerados com as malhas iniciais de elementos finitos, calcula-se a matriz de condutividade que tem forma esparsa. De forma similar, com os dados das matrizes $c d b . d a t$ e nodesb.dat gerados com as malhas renumeradas de elementos finitos, calcula-se a matriz de condutividade diagonal de banda e constrói-se a forma compacta desta matriz de condutividade. Na Tabela 5.1 observam-se as dimensões das matrizes de condutividade de cada caso.

Tabela 5.1: Dimensões das matrizes de condutividade, nas formas esparsa e compacta para os cinco casos de estudo

\begin{tabular}{|l||c|c|c|c|c|}
\hline \hline Caso No. & No. elem. & No. nós & Dim. $Y$ & Dim. $Y c$ & $\triangle$ colunas \\
\hline \hline 1 & 4 & 5 & $5 \times 5$ & $5 \times 5$ & 0 \\
\hline 2 & 32 & 25 & $25 \times 25$ & $25 \times 19$ & 6 \\
\hline 3 & 96 & 65 & $65 \times 65$ & $65 \times 33$ & 32 \\
\hline 4 & 118 & 92 & $92 \times 92$ & $92 \times 55$ & 37 \\
\hline 5 & 182 & 156 & $156 \times 156$ & $156 \times 77$ & 79 \\
\hline \hline
\end{tabular}

onde

- Dim $Y$ é a dimensão da matriz de condutividade esparsa, obtida com a malha inicial para cada caso;

- Dim $Y c$ é a dimensão da matriz de condutividade diagonal de banda em forma compacta, obtida com a malha renumerada para cada caso;

- $\triangle$ colunas é a diferença entre o número de colunas da matriz de condutividade da malha inicial (matriz esparsa) e o número de colunas da matriz de condutividade da malha renumerada na forma compacta.

O diagrama da Figura 5.10 apresenta o número de colunas da matriz de condutividade obtida com a malha inicial $Y$ (lado esquerdo) e o número de colunas da matriz de condutividade diagonal de banda na forma compacta $Y c$ obtida com a malha renumerada (lado direito) para cada caso, 


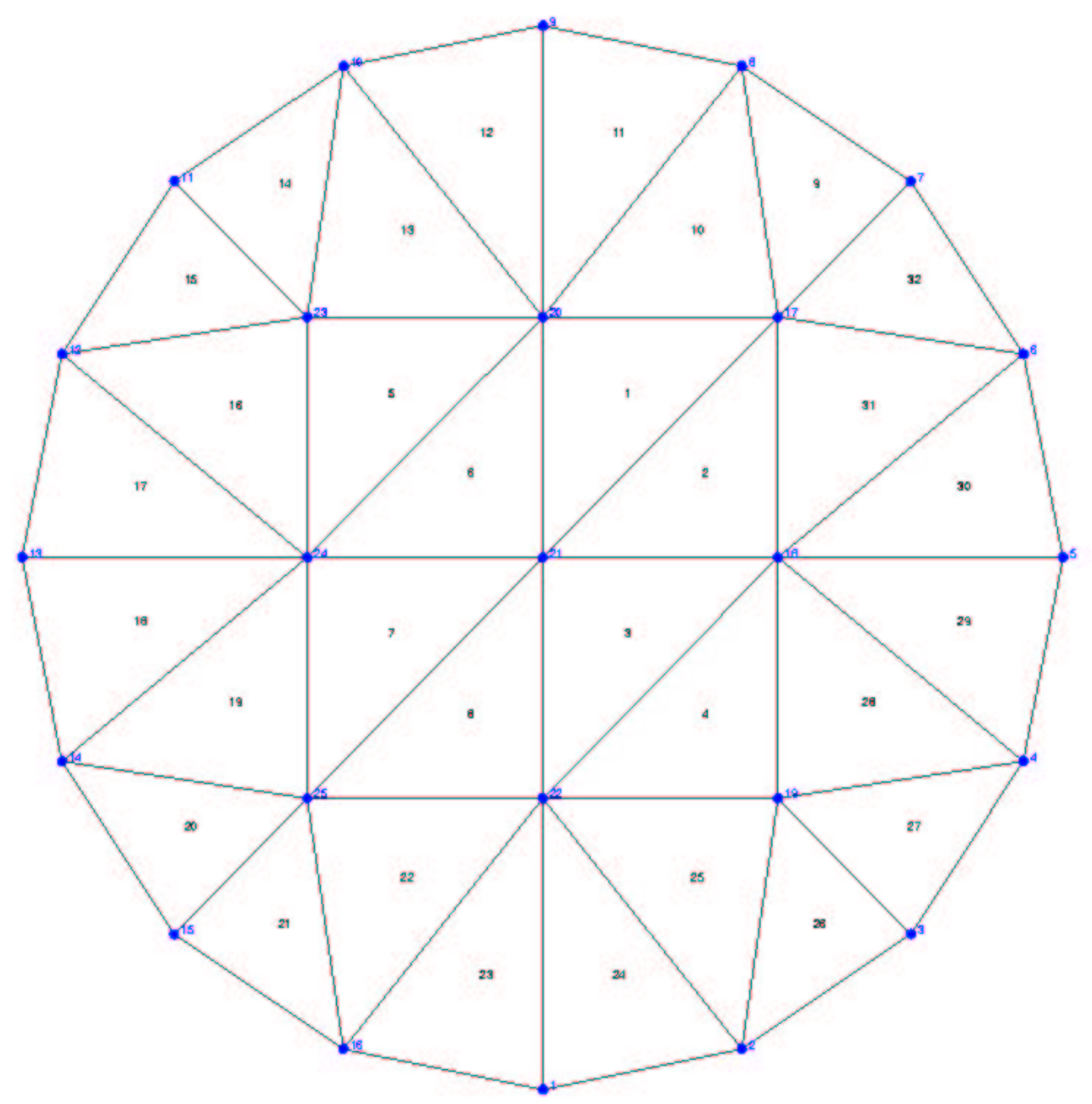

Figura 5.2: Caso 2. Malha inicial de elementos finitos com 32 elementos, 25 nós e 16 eletrodos. 


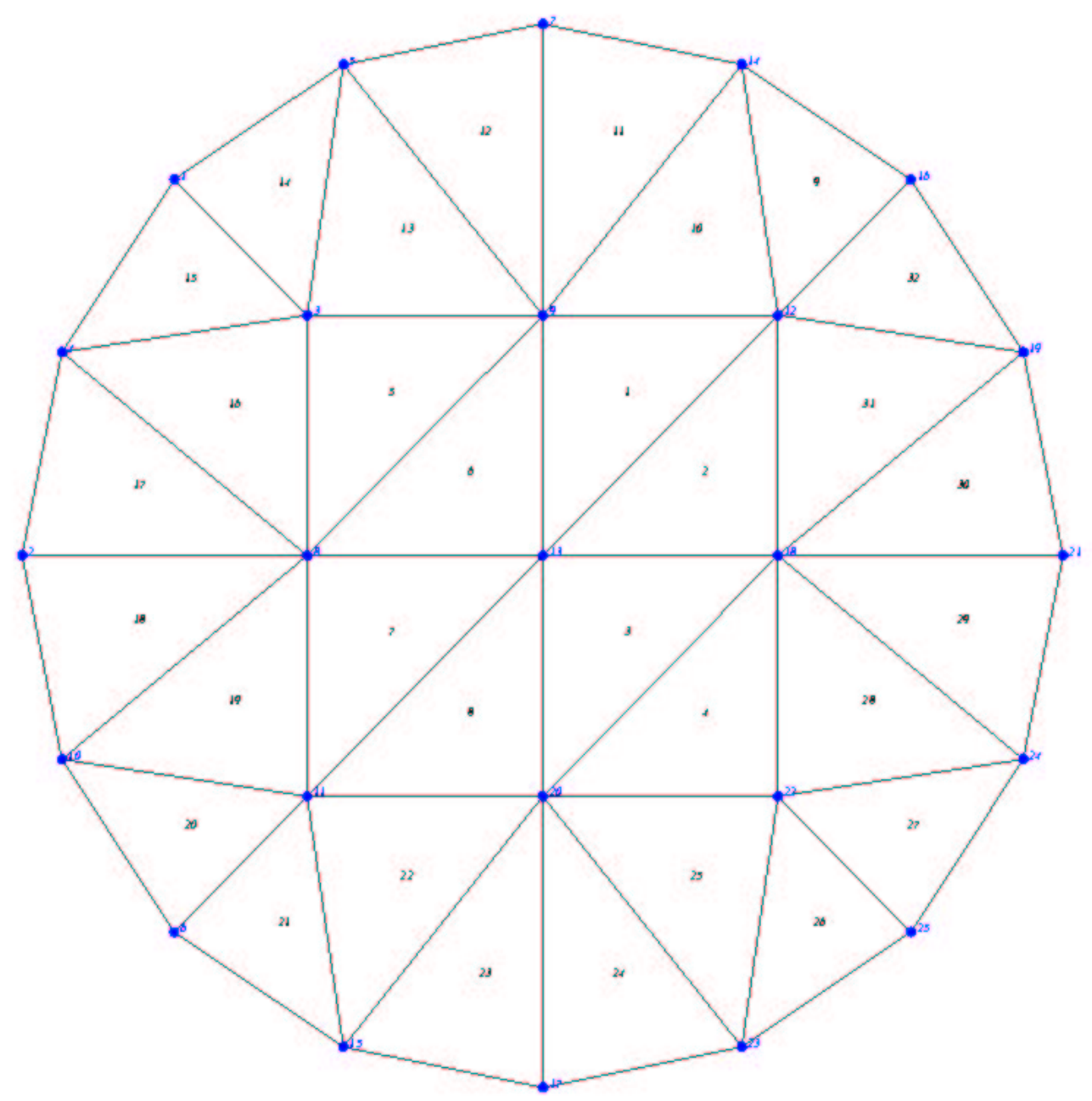

Figura 5.3: Caso 2. Malha renumerada de elementos finitos com 32 elementos, 25 nós e 16 eletrodos. 


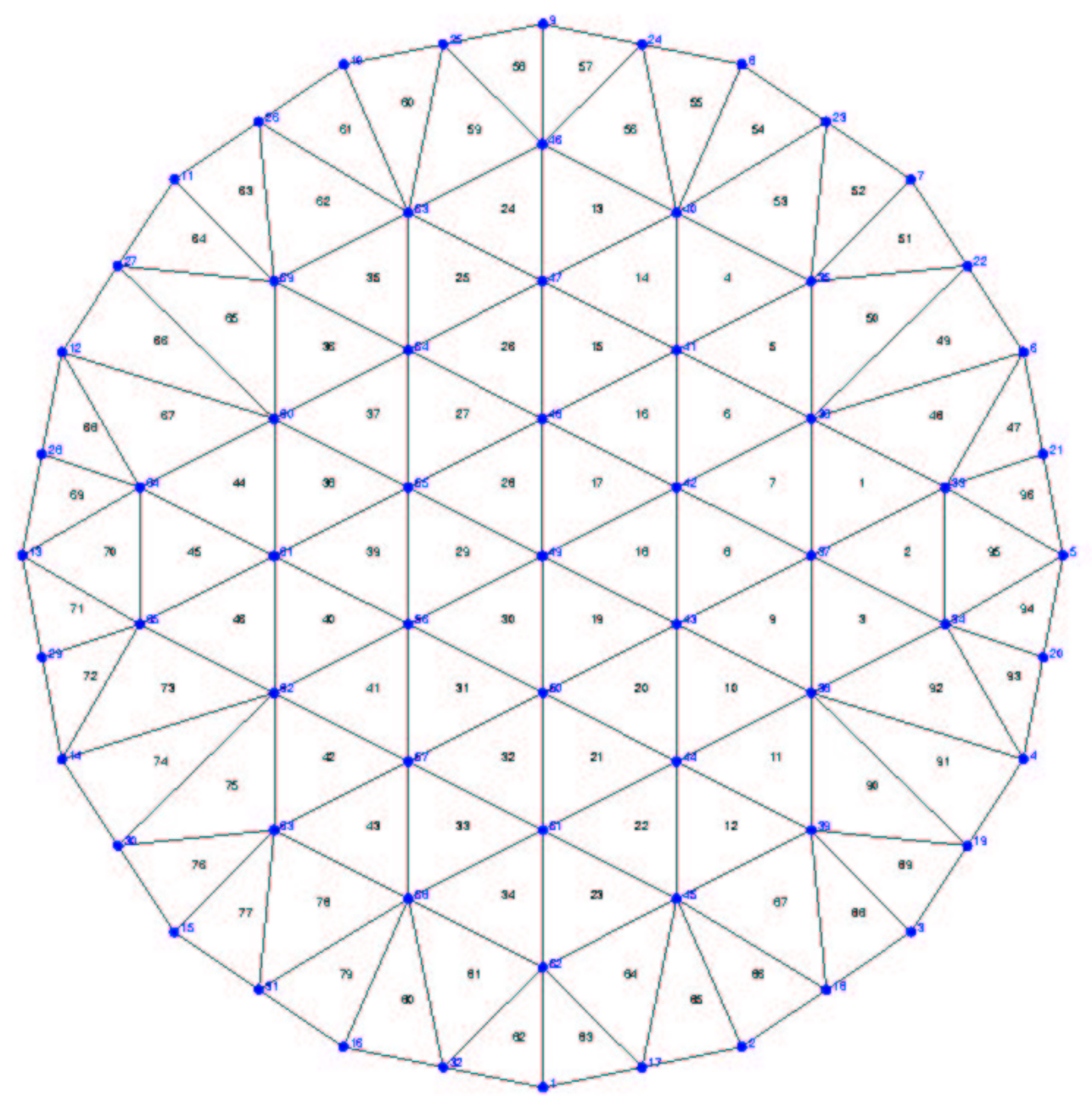

Figura 5.4: Caso 3. Malha inicial de elementos finitos com 96 elementos, 65 nós e 16 eletrodos. 


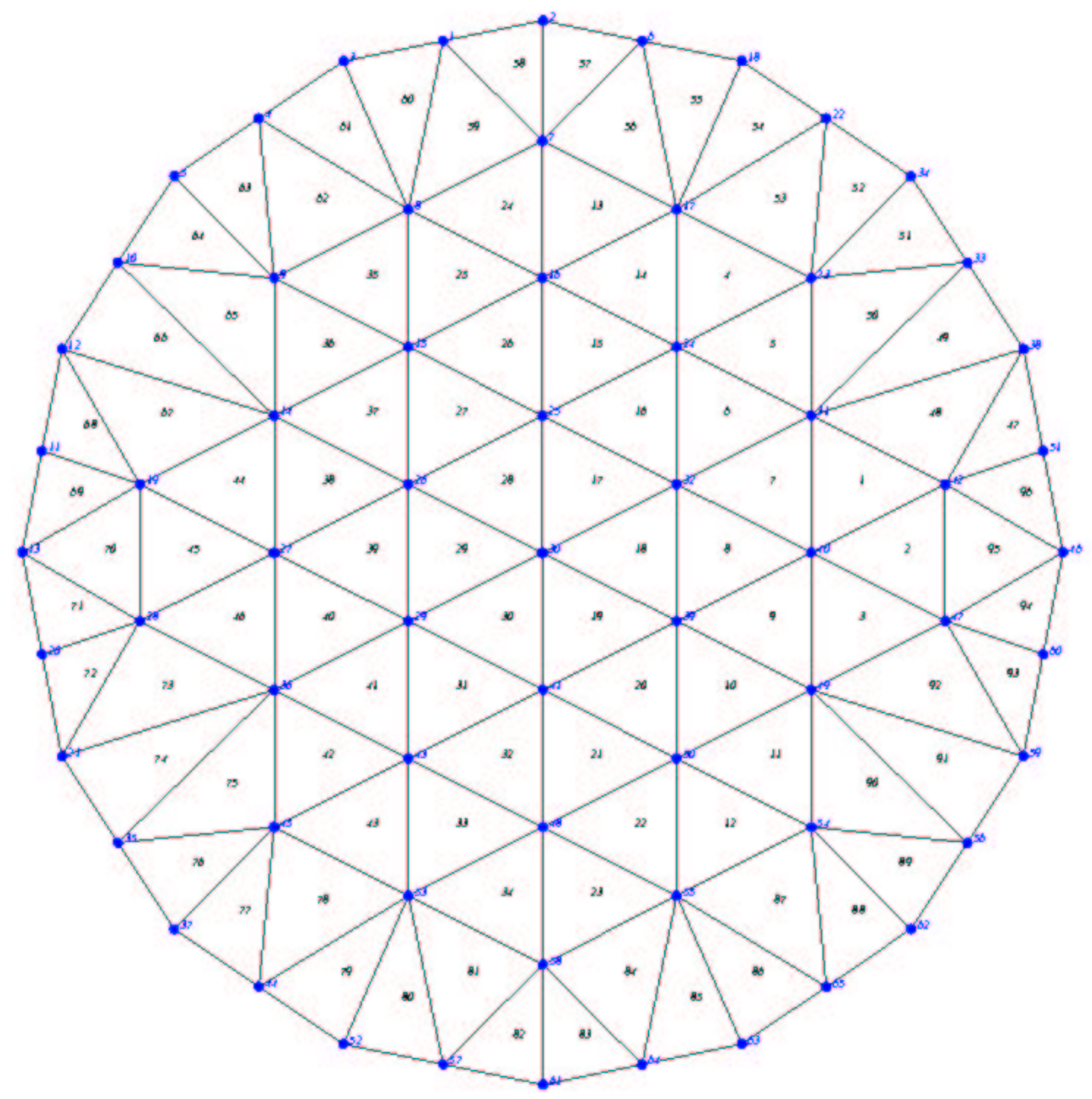

Figura 5.5: Caso 3. Malha renumerada de elementos finitos com 96 elementos, 65 nós e 16 eletrodos. 


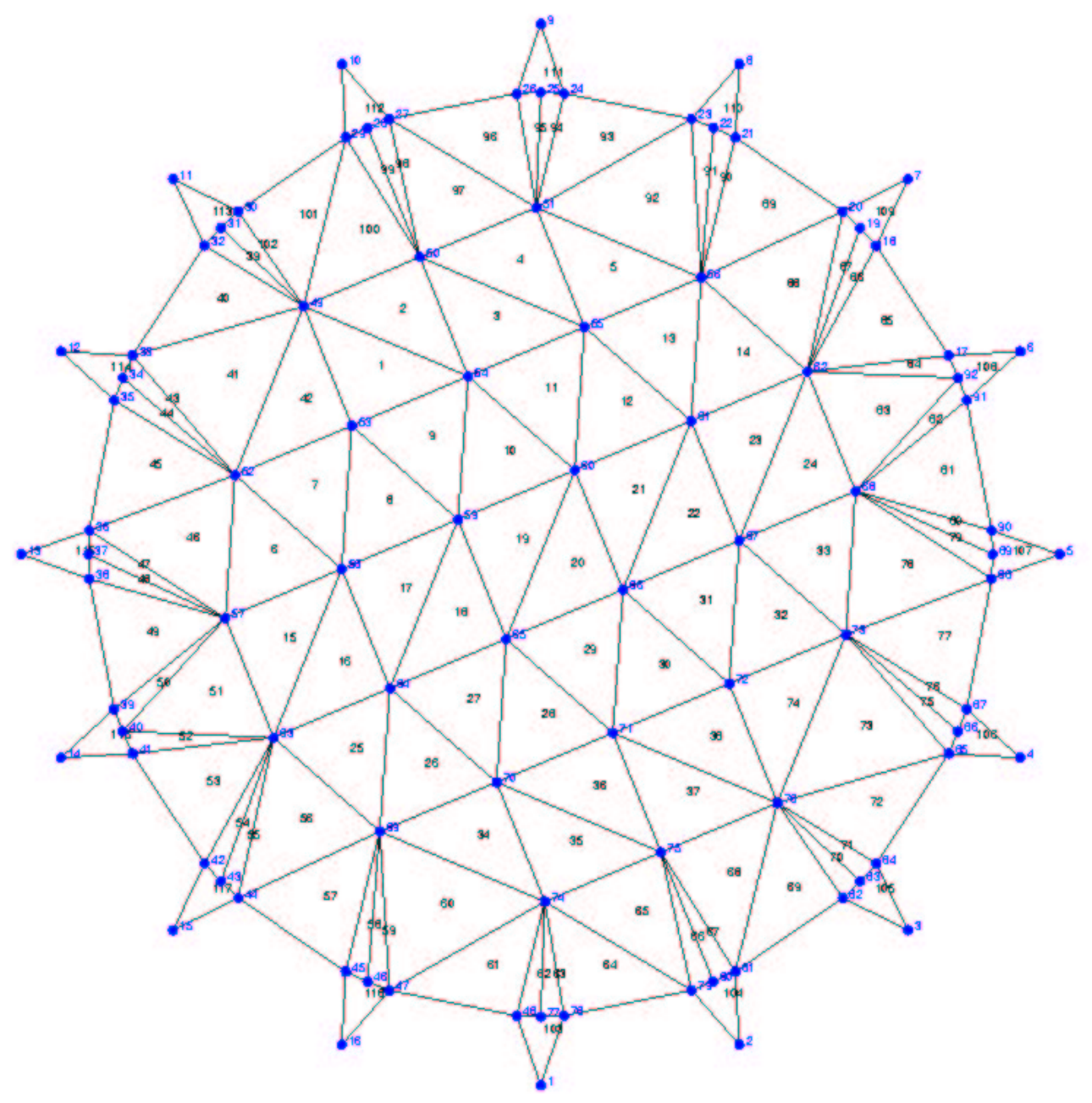

Figura 5.6: Caso 4. Malha inicial de elementos finitos com 118 elementos, 92 nós e 16 eletrodos. 


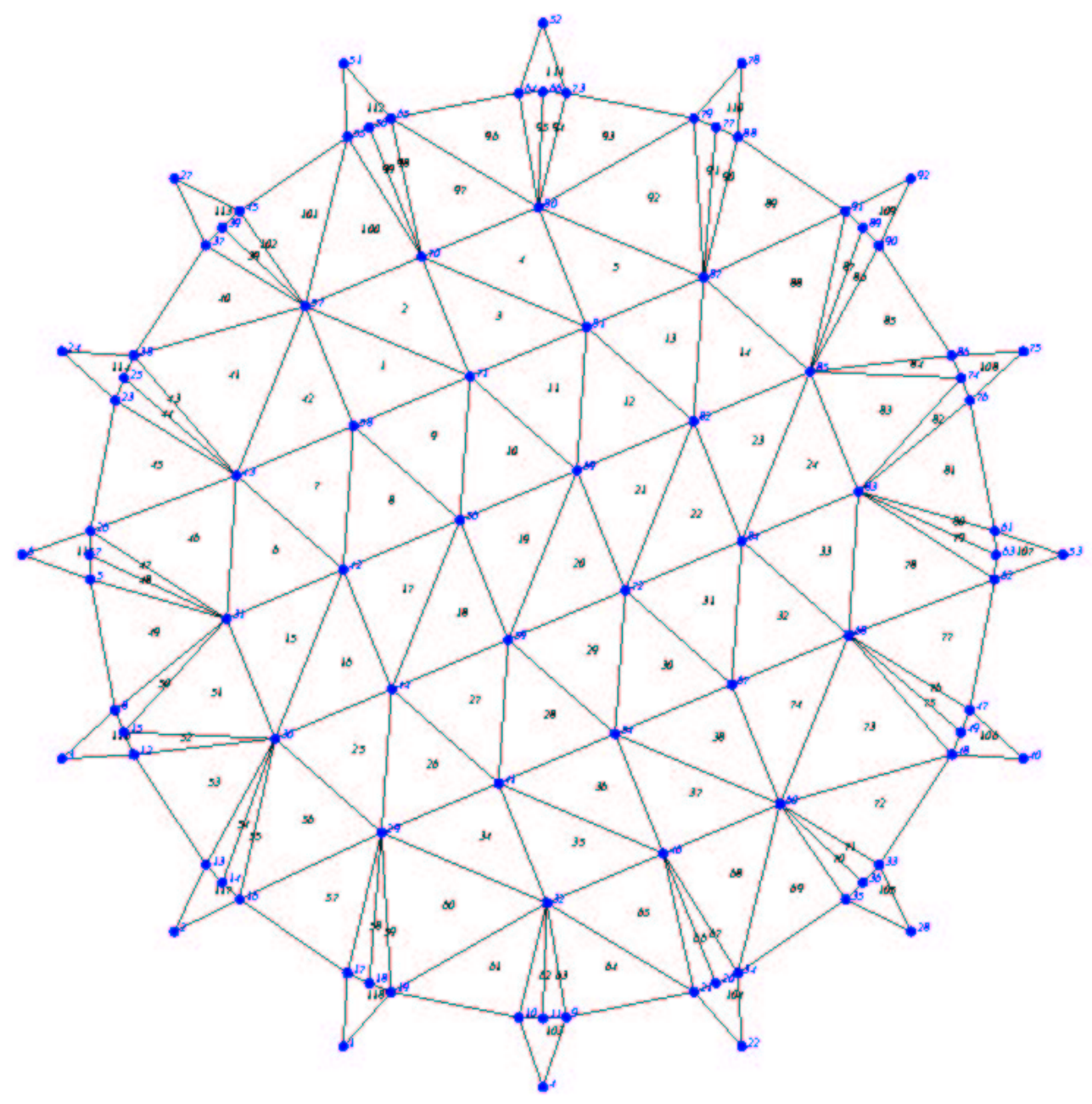

Figura 5.7: Caso 4. Malha renumerada de elementos finitos com 118 elementos, 92 nós e 16 eletrodos. 


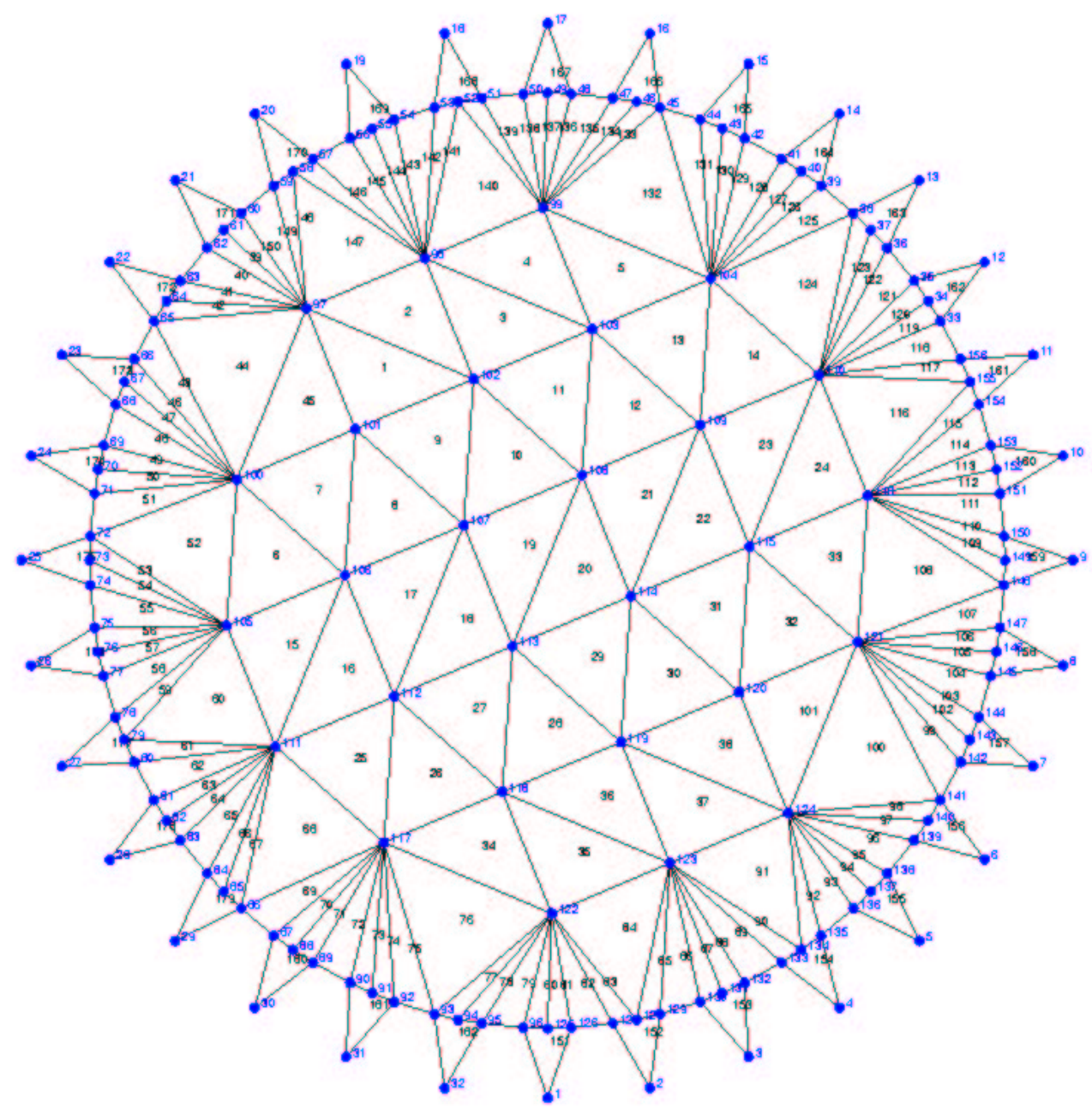

Figura 5.8: Caso 5. Malha inicial de elementos finitos com 182 elementos, 156 nós e 32 eletrodos. 


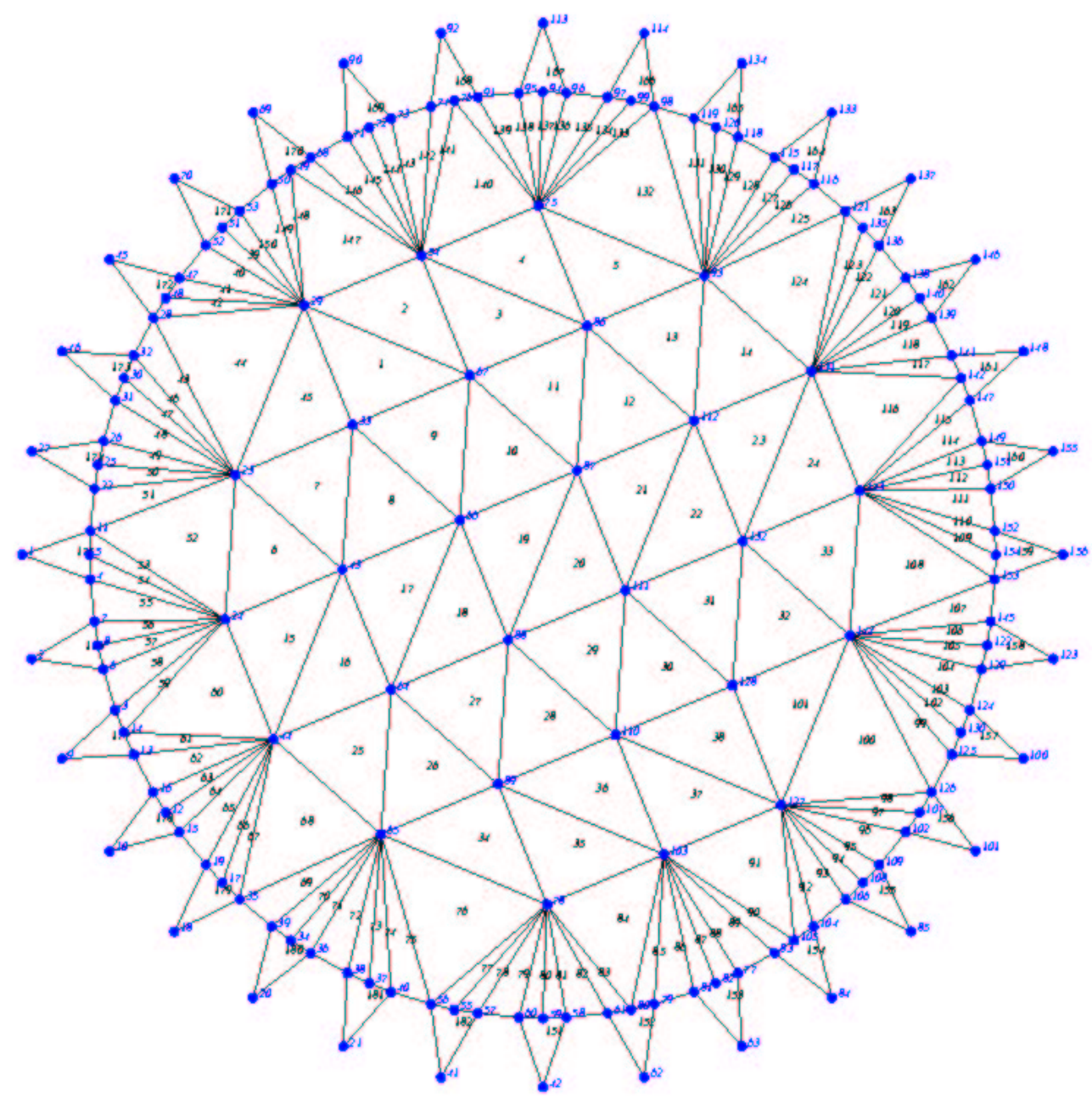

Figura 5.9: Caso 5. Malha renumerada de elementos finitos com 182 elementos, 156 nós e 32 eletrodos. 
sendo que o número de linhas das matrizes não se altera em cada caso.

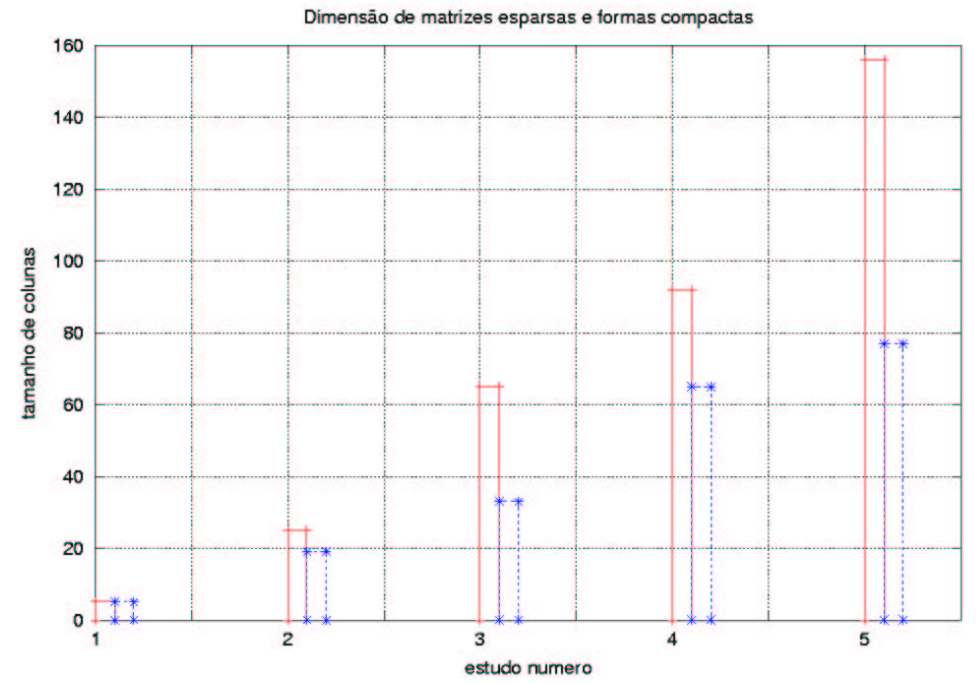

Figura 5.10: Tamanho das colunas das matrizes $Y$ e $Y c$ para os cinco casos de estudo.

Desenvolveu-se um algoritmo em linguagem C, para obter as inversas das matrizes de condutividade $Y$ e $Y c$, aplicando-se na matriz $Y c$ o método mencionado na seção anterior para obter a inversa da matriz de condutividade na forma compacta. Na Tabela 5.2 apresenta-se um resumo dos tempos para o cálculo de cada uma das inversas, onde

Tempo i $Y$ é o tempo em segundos para o cálculo da inversa da matriz de condutividade obtida com a malha inicial, Tempo iYc é o tempo em segundos para o cálculo da inversa da matriz de condutividade diagonal de banda na forma compacta obtida com a malha renumerada e $\triangle \%$ é a percentagem de redução de tempo para obter a inversa da matriz de condutividade para a malha renumerada na forma compacta.

Tabela 5.2: Tempo em segundos para o cálculo da inversa da matriz de condutividade

\begin{tabular}{|l||c|c|c|c|c|}
\hline \hline Caso No. & No. elem. & No. nós & Tempo $i Y(\mathrm{~s})$ & Tempo $i Y c(\mathrm{~s})$ & $\triangle \%$ \\
\hline \hline 1 & 4 & 5 & 0.01 & 0.01 & 0 \\
\hline 2 & 32 & 25 & 0.02 & 0.02 & 0 \\
\hline 3 & 96 & 65 & 0.04 & 0.03 & 25 \\
\hline 4 & 118 & 92 & 0.07 & 0.06 & 14.28 \\
\hline 5 & 182 & 156 & 0.24 & 0.18 & 25 \\
\hline \hline
\end{tabular}

O diagrama de colunas da Figura 5.11 apresenta uma comparação entre Tempo iY e Tempo $i Y c$ em cada caso. 


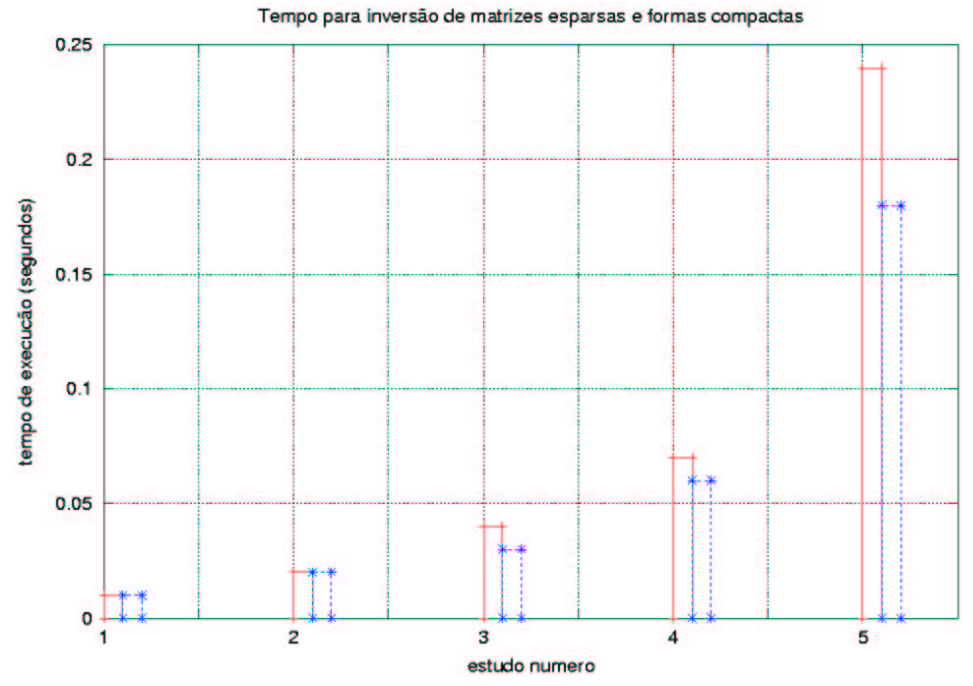

Figura 5.11: Tempo em segundos para o cálculo da inversa da matriz de condutividade.

O algoritmo principal desenvolvido neste trabalho utiliza a malha renumerada conforme mostra a Figura 5.9 (caso 5), ou seja, malha que possui $n e=182$ elementos e $n n=156$ nós, dos quais $n f=32$ correspondem aos eletrodos. Esta malha permite melhor resolução espacial nas imagens. 


\section{Capítulo 6}

\section{REFINAMENTO ITERATIVO}

A solução numérica de sistemas lineares pode ser afetada pelo mal condicionamento do sistema, assim como pelos erros nos cálculos que se fazem com precisão limitada, erros de arredondamento. Logo, a solução obtida não é a solução exata do sistema (embora esteja próxima caso o sistema seja bem condicionado). É preciso controlar tecnicamente essas deficiências e saber quanto está errada a solução. Assim pode-se obter um processo iterativo em que se tenta melhorar a solução calculada por um determinado método.

Obviamente não é fácil obter grande precisão para a solução de um sistema linear, além do limite da precisão da máquina, devido aos métodos diretos de solução redondeiam os erros os quais são acumulados e amplificados na medida em que a matriz é singular.

Mas existe uma forma estruturada de restaurar a precisão máxima da máquina, chamada refinamento iterativo ou melhoramento iterativo da solução. Este procedimento é descrito a seguir [29].

Suponha que o vetor $x$ é a solução exata do sistema linear

$$
A \cdot x=b .
$$

No entanto, não se conhece a solução $x$, mas apenas se conhece uma solução perturbada $x+\delta x$, onde $\delta x$ é a propagação de erro desconhecido. Quando se multiplica a matriz $A$ pela solução perturbada, obtém-se como resultado um vetor ligeiramente diferente de $b$, assim:

$$
A \cdot(x+\delta x)=b+\delta b .
$$

Subtraindo a equação 6.1 da equação 6.2 , resulta : 


$$
A . \delta x=\delta b
$$

Isolando $\delta b$ na equação 6.2 e substituindo a equação 6.3 , decorre

$$
A . \delta x=A .(x+\delta x)-b .
$$

Nesta equação, o termo do lado direito é totalmente conhecido, já que $x+\delta x$ é a solução perturbada que se quer melhorar. É esencial calcular o termo do lado direito com precisão dupla já que haverá uma grande quantidade de cancelações na subtração de $b$. Então precisa-se resolver a equação 6.4 para o erro $\delta x$.

A nova solução estimada é a estimativa inicial $(x+\delta x)$ menos a propagação de erro $(\delta x)$ obtida na equação 6.4 .

Para identificar se o sistema é mal condicionado é preciso calcular o número de condição da matriz $A$. Um número de condição muito elevado indica que o sistema é mal condicionado e, conseqüentemente, o sistema é de difícil solução [37]. Na implementação feita neste estudo o sistema foi resolvido utilizando a decomposição LU.

\subsection{Aplicação do Refinamento Iterativo}

Como foi exposto na solução do problema inverso, uma das matrizes mais relevantes na determinação da matriz $H$ é a matriz de condutividade $Y$ e a sua inversa $Y^{-1}$, cuja solução é uma estimativa com erros de propagação. É objetivo desta seção expôr a forma como foi implementado o procedimento do refinamento iterativo para o cálculo da inversa da matriz de condutividade.

Suponha que o vetor $y_{i}$ é a solução exata do sistema linear,

$$
Y . y_{i}=I_{i}
$$

onde

$Y$ : matriz de condutividade;

$y_{i}$ : vetor solução, coluna $i$ da inversa da matriz de condutividade;

$I_{i}$ : coluna $i$ da matriz identidade.

No entanto, não se conhece a solução de $y_{i}$, mas se conhece a solução perturbada $y_{i}+\delta y_{i}$, onde $\delta y_{i}$ é a propagação de erro desconhecida, calcula-se $\delta y_{i}$ para depois subtraí-lo da solução perturbada a fim de obter uma solução melhorada, 


$$
Y \delta y_{i}=Y \cdot\left(y_{i}+\delta y_{i}\right)-I_{i}
$$

A nova solução estimada é a estimativa inicial $\left(y_{i}+\delta y_{i}\right)$ menos a propagação de erro $\left(\delta y_{i}\right)$ obtida na equação 6.6. Esta é a coluna $i$ da inversa da matriz de condutividade. Portanto, para obter as colunas da inversa da matriz de condutividade repete-se o processo para $i=1, \ldots, n n$, onde $n n$ é o número de nós da matriz de condutividade.

Neste trabalho foi desenvolvido e implementado um algoritmo em linguagem C, para a aplicação do refinamento iterativo na obtenção da inversa da matriz de condutividade. O refinamento iterativo é aplicado somente uma vez em cada iteração, na obtenção da inversa da matriz de condutividade, a qual é melhorada e logo utilizada nos diferentes cálculos da matriz de sensibilidade.

O código fonte do algoritmo, encontra-se no Apêndice B. 


\section{Capítulo 7}

\section{RESULTADOS E DISCUSSÃO}

Neste capítulo apresentam-se os resultados obtidos com o Filtro Estendido de Kalman, desenvolvido em linguagem $\mathrm{C}$, implementado em duas versões

- versão 1. Denominado Algoritmo inicial com matriz de condutividade esparsa (Alg_Esparsa) malha discretizada com a geometria que representa o phantom, obtida com o módulo de geração automática de malhas Corduroy que faz parte do software FElt;

- versão 2. Denominado Algoritmo com malha renumerada e refinamento iterativo (Alg_RRI). No qual é empregado o módulo de renumeração de malha e implementado o refinamento iterativo para obter a inversa da matriz de condutividade.

Todos os resultados são obtidos a partir da avaliação do algoritmo com os dados experimentais adquiridos na bancada 3 apresentada na seção 3.2.4 e Figura 3.12, composto por um objeto cilíndrico de vidro de diâmetro $6 \mathrm{~cm}$ dentro de um phantom, P-3, de diâmetro $22 \mathrm{~cm}$, contendo solução salina (1300 gramas de água destilada por 0.300 gramas de $\mathrm{NaCl}$ ). O objetivo é estimar a posição do objeto cilíndrico de vidro e seu respectivo valor de impedância, a partir das voltagens medidas nos 32 eletrodos.

A Figura 7.1 apresenta a malha de elementos finitos que representa o phantom (P-3) da bancada experimental 3 (B-3) com a posição do objeto cilíndrico de vidro, empregada na avaliação do algoritmo do Filtro Estendido de Kalman neste capítulo.

Para a aplicação dos algoritmos desenvolvidos agrupam-se os elementos da malha de elementos finitos em diferentes regiões de interesse (ROIs). Através deste agrupamento de elementos melhora a estabilidade das soluções.

Num modelo do tórax, estas ROIs devem ter semelhança geométrica com as estruturas reais das regiões existentes.

Inicialmente formam-se 33 ROIs na malha de elementos finitos para estimar os parâmetros 


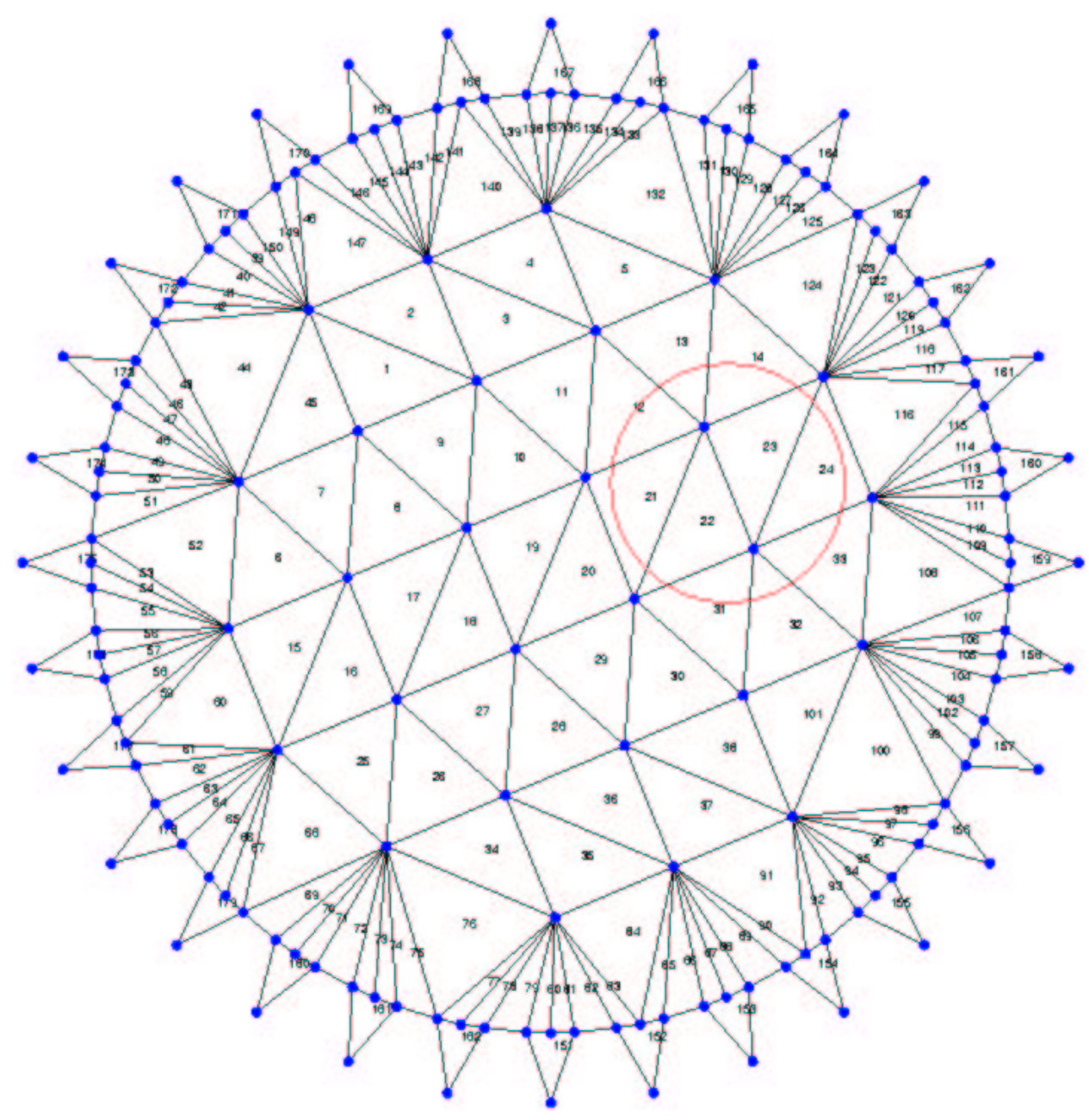

Figura 7.1: Malha de elementos finitos do phantom com 182 elementos, 156 nós e 32 eletrodos. Phantom de diâmetro $22 \mathrm{~cm}$ com o objeto cilíndrico de vidro de diâmetro $6 \mathrm{~cm}$. 
dos 32 eletrodos. Posteriormente, identifica-se o objeto cilíndrico de vidro na malha com 5,8 ou 16 ROIs utilizando as duas versões do filtro. Para avaliar o desempenho do algoritmo, a forma de agrupar as regiões de interesse não guarda estreita semelhança geométrica com o objeto a estimar.

A metodologia utilizada para obter os diferentes resultados inclue :

- geração automática da malha de elementos finitos, malha com 182 elementos, 156 nós e 32 eletrodos;

- obtenção dos parâmetros dos 32 eletrodos, agrupando-se os elementos da malha de elementos finitos em 33 ROIs, utilizando dados experimentais obtidos através de o padrões de corrente diametrais;

- agrupamento dos elementos da malha em 5, 8 ou 16 ROIs e posterior estimação das impedâncias em cada região, utilizando dados experimentais obtidos através de padrões de corrente diametrais;

- geração dos seguintes gráficos : gráfico de contorno da distribuição de impedância; gráfico tridimensional da impedância; gráfico do resíduo normalizado para a verificação da convergência. Os gráficos foram gerados com o software Gnuplot;

- Para comparar os diferentes algoritmos, utilizaram-se o mesmo número de iterações e as mesmas matrizes de covariância no Filtro de Kalman para um dado agrupamento (5, 8 ou 16 ROIs). Na Tabela 7.1 apresenta-se um resumo dos três agrupamentos e as matrizes de covariância utilizados no filtro de Kalman, onde:

No. ROIs é o número de regiões de interesse para a malha de elementos finitos;

$P_{o}$ é a condição inicial da matriz de covariância do erro de estimação;

$R$ é matriz de covariância do ruído nas medidas;

$I_{5}, I_{8}, I_{16}, I_{32}$ : matriz de identidade de ordem $5,8,16$ e 32 respectivamente;

$\hat{\rho}_{(o)}^{(+)}$: condição inicial do vetor de impedâncias para os elementos. Nos três tipos de agrupamentos foi utilizado o mesmo vetor como condição inicial.

As matrizes $P_{o}$ e $R$, são obtidas por experimentação numérica.

É importante mencionar que os parâmetros $\left(P_{o}^{(+)}\right.$e $\left.Q\right)$ aplicados no Filtro de Kalman aos três agrupamentos estudados foram obtidos de acordo com Brogan [6], conforme seção 4.3.12,

$P_{o}^{(+)}=\left[P_{o}^{-1}+H^{t} R^{-1} H\right]^{-1}$

$Q=0.001 * P_{o}^{(+)}$

onde

$P_{o}^{(+)}$: estimativa inicial da matriz de covariância do erro de estimação;

$Q$ : matriz de covariância do ruído no estado. 
Tabela 7.1: Resumo dos três agrupamentos estudados e parâmetros do Filtro Estendido de Kalman para 5, 8 ou 16 ROIs na malha de elementos finitos com 182 elementos, 156 nós e 32 eletrodos

\begin{tabular}{|l||c|c|c|c|c|}
\hline \hline Agrupamento & No. ROIs & Versão No. & Malha & $P_{o}$ & $R$ \\
\hline \hline \multirow{2}{*}{1} & 5 & 1 & Figura 5.8 & $2.0 I_{5}$ & $9.0 \mathrm{e}-02 I_{32}$ \\
\cline { 3 - 6 } & & 2 & Figura 5.9 & $2.0 I_{5}$ & $9.0 \mathrm{e}-02 I_{32}$ \\
\hline \multirow{2}{*}{2} & 8 & 1 & Figura 5.8 & $2.0 I_{8}$ & $9.0 \mathrm{e}-02 I_{32}$ \\
\cline { 3 - 6 } & & 2 & Figura 5.9 & $2.0 I_{8}$ & $9.0 \mathrm{e}-02 I_{32}$ \\
\hline \multirow{2}{*}{3} & 16 & 1 & Figura 5.8 & $0.4 I_{16}$ & $9.0 \mathrm{e}-02 I_{32}$ \\
\cline { 3 - 6 } & & 2 & Figura 5.9 & $0.4 I_{16}$ & $9.0 \mathrm{e}-02 I_{32}$ \\
\hline \hline$\hat{\rho}_{(o)}^{(+)}$ & $11.111(\Omega m)$ elementos: $1,2, . .38 . \mathrm{e} 45,101$ \\
\cline { 2 - 6 } & $6.666(\Omega m)$ o resto de elementos \\
\hline \hline
\end{tabular}

Os valores das condutividades têm unidades $(\Omega m)^{-1}$ e os valores das impedâncias têm unidades $(\Omega m)$.

No Apêndice C, apresentam-se detalhadamente os algoritmos de estimação desenvolvidos para TIE escritos inicialmente com o software Octave e posteriormente em linguagem C.

\subsection{Estimativa das Condutividades nos 32 Eletrodos}

O passo prévio para estimativar a impedância das ROIs consiste em obter uma estimativa da condutividade nos 32 eletrodos. Para isto foi utilizada a malha de elementos finitos de 182 elementos agrupados em 33 ROIs, onde cada uma das 32 regiões são compostas por cada um dos eletrodos e uma região para os demais elementos (150 elementos) da malha.

Utilizou-se o modelo completo de eletrodos, apresentado pelo pesquisador Hua Ping et al. no artigo Finite Element Modeling of Electrode-Skin Contact Impedance in Electrical Impedance Tomography [20]. O modelo leva em consideração efeitos como a impedância de contato entre a pele e a superfície metálica do eletrodo e as dimensões dos eletrodos que podem chegar a ocupar quase a metade do perímetro do tórax estudado. Neste trabalho são usados eletrodos largos que cobrem $80 \%$ do perímetro do phantom. Estes eletrodos fornecem uma densidade de corrente baixa e baixa impedância de contato pois a impedância de contato é inversamente proporcional à área de contato.

Na Tabela 7.2 apresentam-se os parâmetros empregados no algoritmo do Filtro Estendido de Kalman para obter as estimativas de condutividades nos 32 eletrodos. 
Tabela 7.2: Parâmetros utilizados para obter uma estimativa da condutividade nos 32 eletrodos

\begin{tabular}{|l||c|}
\hline \hline Parâmetro & Valor \\
\hline \hline$P_{o}$ & $3.5 \mathrm{e}-01 I_{33}$ \\
\hline$R$ & $2.5 \mathrm{e}-05 I_{32}$ \\
\hline \multirow{2}{*}{$\hat{\sigma}_{(o)}^{(+)}$} & $10.0(\Omega m)^{-1}$ para os elementos: $151,152, . ., 182$. (eletrodos) \\
\cline { 2 - 2 } & $0.06(\Omega m)^{-1}$ para os elementos restantes \\
\hline \hline
\end{tabular}

onde

$P_{o}:$ condição inicial da matriz de covariância do erro de estimação;

$R$ : matriz de covariância do ruído nas medidas;

$I_{32}, I_{33}$ são matrizes de identidade de ordem 32 e 33, respectivamente;

$\hat{\sigma}_{(o)}^{(+)}$é a condição inicial do vetor de condutividades para os elementos.

Os parâmetros apresentados na Tabela 7.2 foram obtidos por experimentação numérica buscando melhor convergência. Aplicou-se o padrão de corrente diametral para obter os dados experimentais das voltagens nos eletrodos na ausência de objetos no recipiente. A Figura 7.2 apresenta a história dos parâmetros estimados nas 33 ROIs.

Foram utilizados os valores dos parâmetros obtidos na iteração 3200 embora nem todos os valores tenham atingido convergência. Os valores estimados das 32 primeiras ROIs correspondem aos parâmetros dos modelos dos 32 eletrodos. O recíproco destes valores serão utilizados na estimativa da distribuição de impedâncias.

$\mathrm{Na}$ Tabela 7.3 resumem-se os valores nos 32 eletrodos, estimados a partir das leituras de voltagens medidas nos 32 eletrodos e da implementação do modelo completo de eletrodos no Filtro Estendido de Kalman.

O algoritmo para estimar os valores nos eletrodos foi desenvolvido e verificado em função de $1 / \rho_{t}$ ao invés de $\rho_{t}$ por ter apresentado melhor estabilidade.

Finalmente, as estimativas de $1 / \rho_{t}$ dos eletrodos obtidas são convertidas em $\rho_{t}$ utilizadas na estimativa da distribuição de impedância. A impedância da região 33 que corresponde à solução salina $17 \Omega m$. 
Tabela 7.3: Estimativas de parâmetros nos 32 eletrodos, obtidas com a malha agrupada em 33 ROIs a partir de dados experimentais

\begin{tabular}{|c|c|c|c|c|}
\hline ROIs No. & Eletrodo No. & Elemento No. & $1 / \rho_{t}$ & $\rho_{t}$ \\
\hline 1 & 1 & 151 & 23.0871 & 0.0433143 \\
\hline 2 & 2 & 152 & 11.3094 & 0.0884223 \\
\hline 3 & 3 & 153 & 12.6523 & 0.0790371 \\
\hline 4 & 4 & 154 & 18.5358 & 0.0539496 \\
\hline 5 & 5 & 155 & 27.9147 & 0.0358234 \\
\hline 6 & 6 & 156 & 35.4838 & 0.0281819 \\
\hline 7 & 7 & 157 & 27.6568 & 0.0361575 \\
\hline 8 & 8 & 158 & 38.5945 & 0.0259104 \\
\hline 9 & 9 & 159 & 68.2166 & 0.0146592 \\
\hline 10 & 10 & 160 & 104.241 & 0.00959311 \\
\hline 11 & 11 & 161 & 138.845 & 0.00720229 \\
\hline 12 & 12 & 162 & 145.975 & 0.00685047 \\
\hline 13 & 13 & 163 & 96.1316 & 0.0104024 \\
\hline 14 & 14 & 164 & 136.082 & 0.00734849 \\
\hline 15 & 15 & 165 & 81.3336 & 0.012295 \\
\hline 16 & 16 & 166 & 43.7415 & 0.0228616 \\
\hline 17 & 17 & 167 & 145.975 & 0.00685047 \\
\hline 18 & 18 & 168 & 74.0276 & 0.0135085 \\
\hline 19 & 19 & 169 & 55.3247 & 0.0180751 \\
\hline 20 & 20 & 170 & 60.2654 & 0.0165933 \\
\hline 21 & 21 & 171 & 27.7807 & 0.0359962 \\
\hline 22 & 22 & 172 & 20.4016 & 0.0490157 \\
\hline 23 & 23 & 173 & 19.2491 & 0.0519504 \\
\hline 24 & 24 & 174 & 17.2817 & 0.0578647 \\
\hline 25 & 25 & 175 & 16.7855 & 0.0595754 \\
\hline 26 & 26 & 176 & 13.448 & 0.0743605 \\
\hline 27 & 27 & 177 & 15.1423 & 0.0660404 \\
\hline 28 & 28 & 178 & 13.2147 & 0.0756731 \\
\hline 29 & 29 & 179 & 16.3294 & 0.0612394 \\
\hline 30 & 30 & 180 & 13.1527 & 0.0760302 \\
\hline 31 & 31 & 181 & 12.131 & 0.0824334 \\
\hline 32 & 32 & 182 & 13.7485 & 0.072735 \\
\hline 33 & - & 1 a 150 & $5.8904 \mathrm{e}-02$ & 16.976775 \\
\hline
\end{tabular}




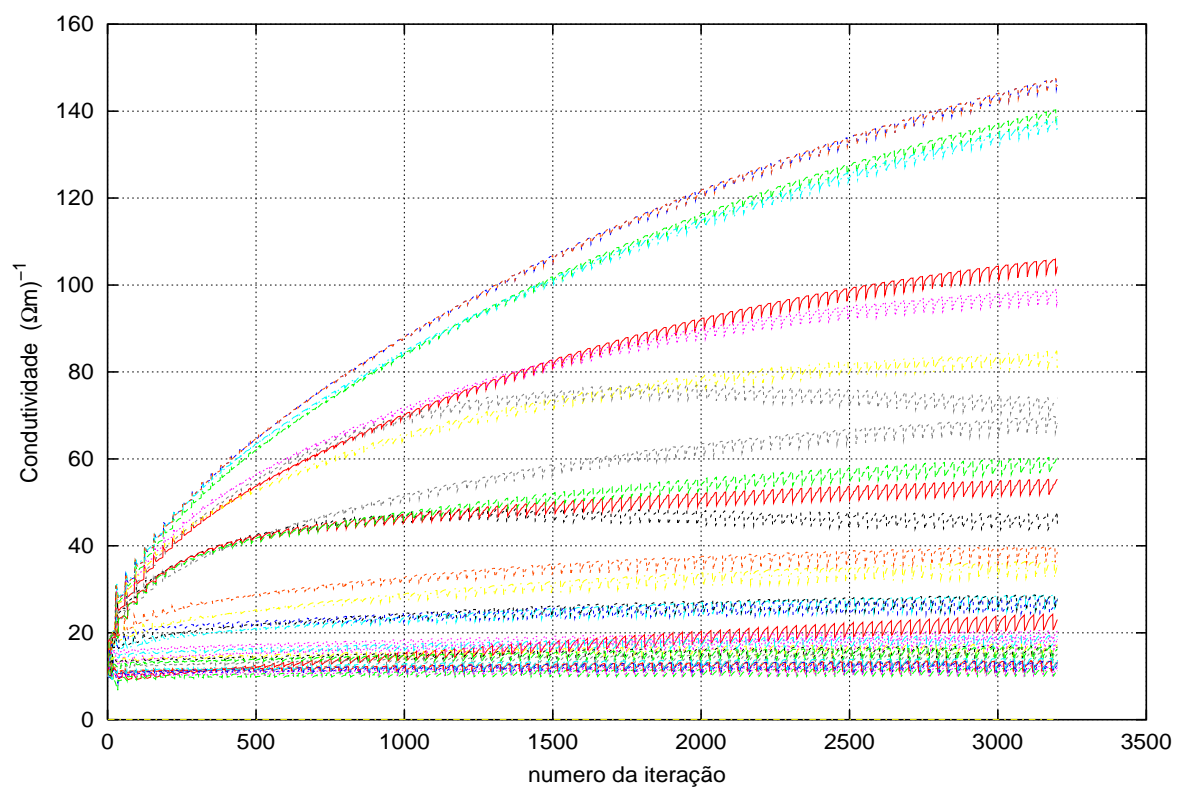

Figura 7.2: Estimativas dos parâmetros no tempo nas 33 ROIs, onde 32 ROIs representam os 32 eletrodos. Para a malha com 182 elementos, 156 nós e 32 eletrodos. Gráfico de 3200 iterações. 


\subsection{Cinco Regiões de Interesse (5 ROIs)}

Na Figura 7.3 mostra-se a discretização do domínio que representa a seção do phantom, em 182 elementos, 156 nós e 32 eletrodos. Agrupam-se em 5 ROIs os elementos da malha de elementos finitos, na qual os últimos 32 elementos da malha são os eletrodos. Na Tabela 7.4 enumeram-se os elementos que constituem as 5 ROIs da malha mostrada na Figura 7.3.

Neste caso quer-se estimar a impeditividade das 5 ROIs para identificar o objeto dentro do phantom o qual se encontra na região número 2.

Tabela 7.4: Elementos que constituem as 5 regiões de interesse para a malha de elementos finitos da Figura 7.3 e parâmetros para o Filtro de Kalman

\begin{tabular}{|l||c|c|c|}
\hline \hline Região No. & $\hat{\rho}_{(o)}^{(+)}(\Omega m)$ & Elementos que constituem a região & Contém \\
\hline \hline 1 & 11.111 & $1,2,3,4,7,8,9,10,19,45$ & S. salina \\
\hline 2 & 11.111 & $5,11,12,13,14,21,22,23,24,33$ & Objeto \\
\hline 3 & 11.111 & $6,15,16,17,18,25,26,27,28,34$ & S. salina \\
\hline 4 & 11.111 & $20,29,30,31,32,35,36,37,38,101$ & S. salina \\
\hline 5 & 6.666 & o resto de elementos & S. salina \\
\hline \hline$P_{o}$ & $2.0 I_{5}$ & \\
\hline$R$ & $9.0 \mathrm{e}-02 I_{32}$ & \\
\hline \hline
\end{tabular}

onde

$\hat{\rho}_{(o)}^{(+)}$: condição inicial do vetor de impedâncias para as regiões;

S. salina : solução salina, estimativa de impedância $17 \Omega m$;

Objeto : objeto cilíndrico de vidro que se encontra dentro do phantom. Segundo dados teóricos [28], a impeditividade para um objeto cilíndrico de vidro de caraterísticas similares ao utilizado é de $10 e+06 \Omega m$ a temperatura ambiente. 


\section{ROIS}
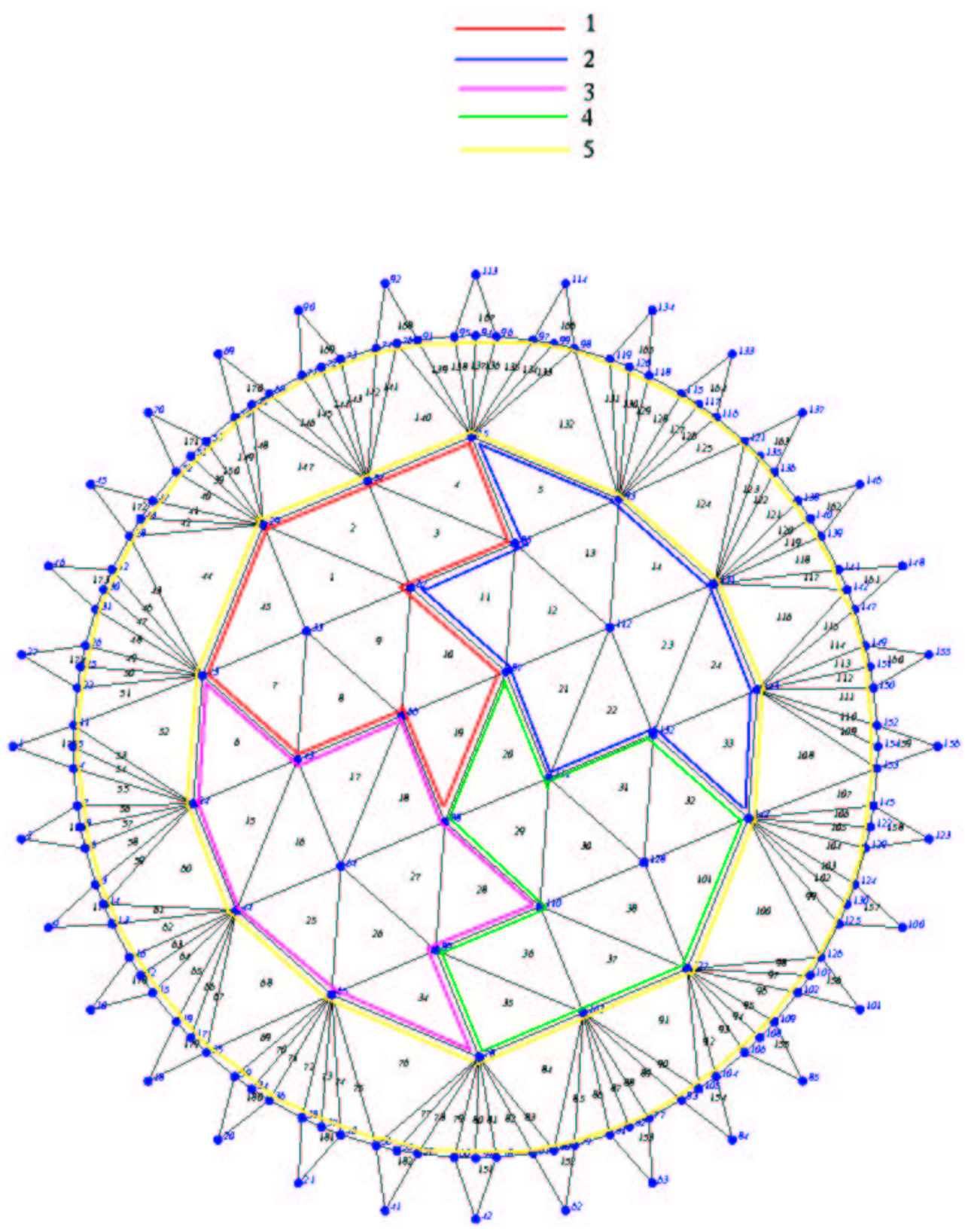

Figura 7.3: Agrupamento 1. 5 regiões de interesse para a malha de elementos finitos que representa o phantom em 182 elementos, 156 nós e 32 eletrodos. Na região número 2 encontra-se o objeto cilíndrico de vidro. 


\subsubsection{Estimativa de Impeditividade em 5 ROIs, Alg_Esparsa}

Com a Malha inicial (Alg_Esparsa) da Figura 5.8 e os parâmetros da Tabela 7.4 foram estimadas as impedâncias para as 5 ROIs conforme o agrupamento dos elementos na Figura 7.3. Para isto foi utilizado o algoritmo desenvolvido para TIE, empregando as estimativas de parâmetros nos eletrodos mostradas na Tabela 7.3. Foram utilizados dados experimentais das voltagens nos eletrodos obtidos através de padrões de corrente diametrais.

Foi gerada a Figura 7.4(a) de impedividade em cada uma das 5 ROIs após 1920 iterações

A Figura 7.4(b) mostra o gráfico tridimensional da impeditividade das 5 ROIs na última iteração após 1920 iterações.

As Figuras 7.5(a) e 7.5(b) apresentem respectivamente, o gráfico de impeditividade em cada uma das 5 ROIs e o gráfico tridimensional das impedâncias das 5 ROIs na última iteração após 12800 iterações.

\subsubsection{Estimativa de Impeditividade em 5 ROIs, Alg_RRI}

Apresentam-se aqui os resultados obtidos com um algoritmo Alg_RRI. Diminuiu-se o erro numérico e obtiveram-se melhores estimativas de impeditividade nas ROIs identificando a posição do objeto.

Geraram-se as seguintes figuras : Figura 7.4(c) de impeditividade nas 5 ROIs e Figura 7.4(d) do gráfico tridimensional da impedância das 5 ROIs na última iteração após 1920 iterações.

Nas Figuras 7.5(c) e 7.5(d) mostra-se respectivamente o gráfico de impeditividade em cada uma das 5 ROIs e o gráfico tridimensional da impedância das 5 ROIs na última iteração após 12800 iterações.

\subsection{Oito Regiões de Interesse (8 ROIs)}

Na Figura 7.6 mostra-se a malha de 182 elementos, 156 nós, 32 eletrodos e como foram agrupados em 8 ROIs os elementos da malha de elementos finitos, onde os últimos 32 elementos da malha são os eletrodos. Na Tabela 7.5 enumeram-se os elementos que constituem cada ROI

Neste caso quer-se estimar a impeditividade das 8 ROIs para identificar o objeto dentro do phantom o qual se encontra entre as regiões de números 2,3 e 4.

Na tabela acima, indica-se por :

- $\hat{\rho}_{(o)}^{(+)}$a condição inicial do vetor de impedâncias para as regiões;

- S. salina a solução salina $17 \Omega m$; 


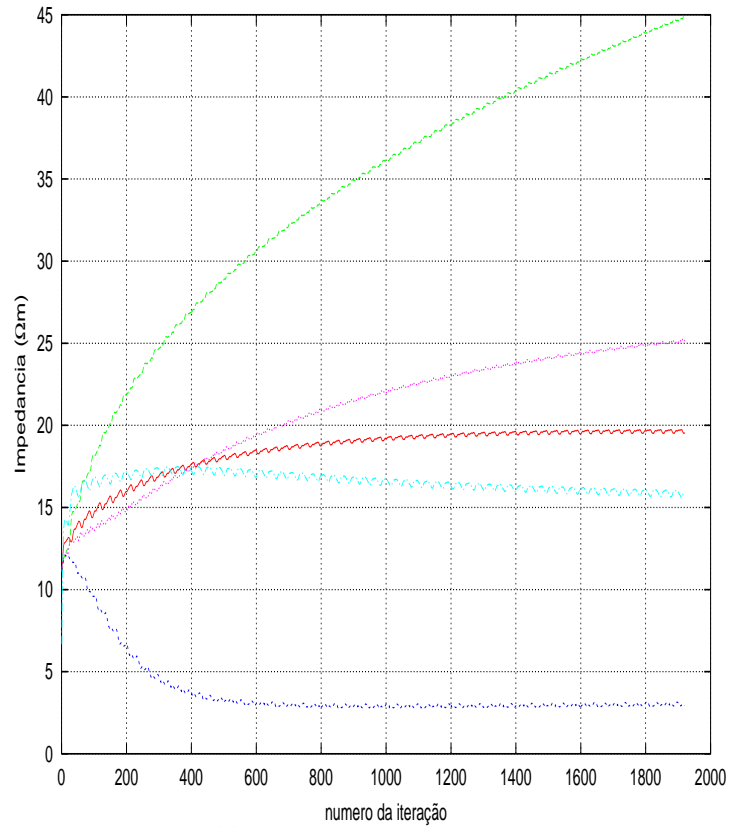

(a)

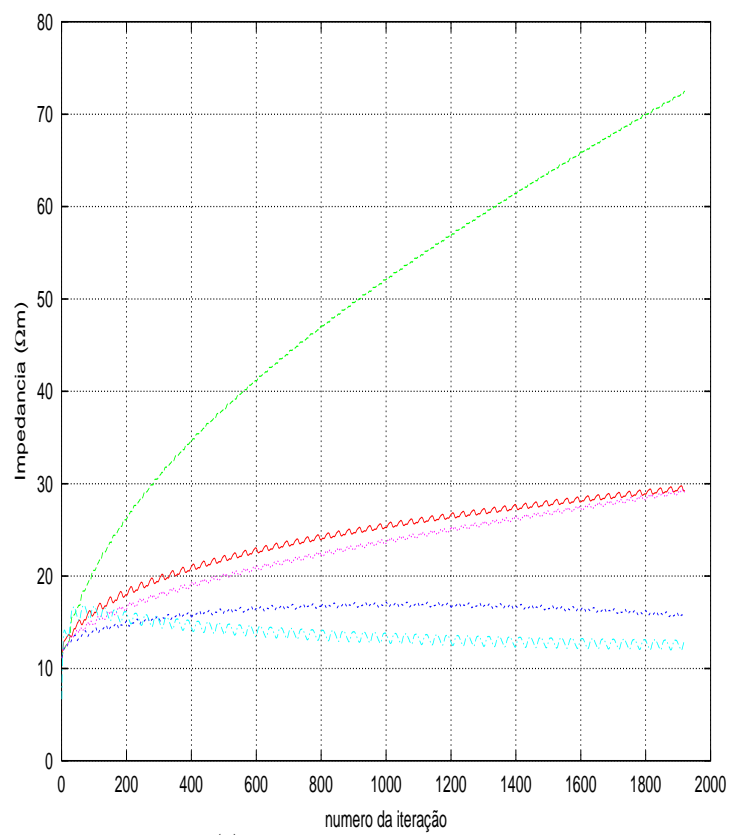

(c)

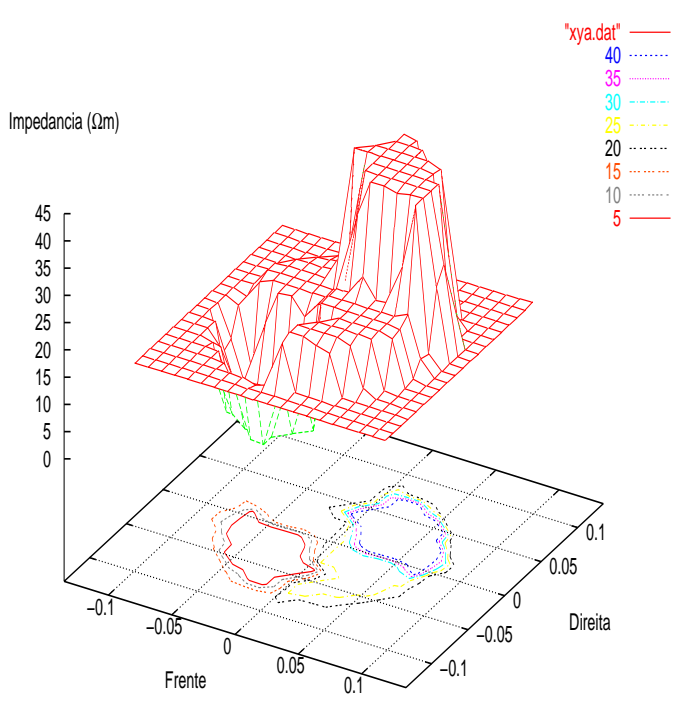

(b)

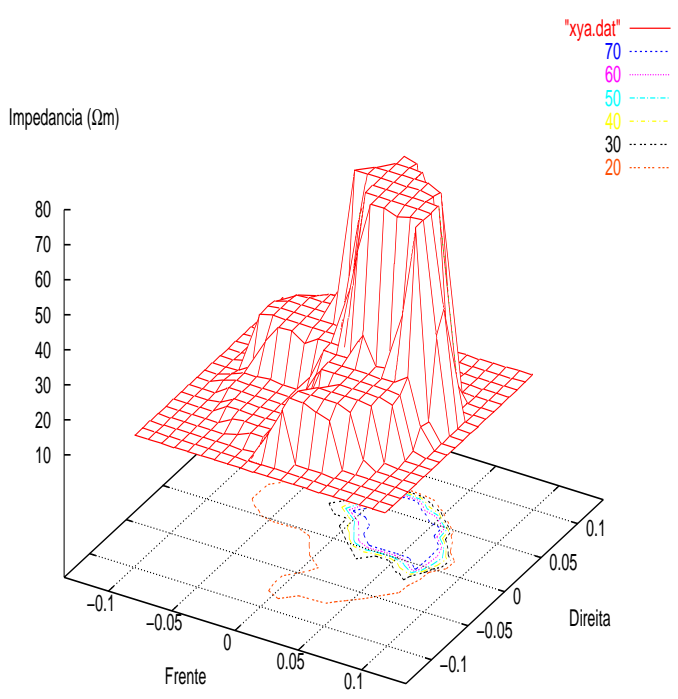

(d)

Figura 7.4: Comparação dos resultados do Agrupamento 1, 5 ROIs. Estimativas de impeditividade no tempo para as 5 ROIs : (a) Alg_Esparsa, (c) Alg_RRI. Representação tridimensional do vetor de impeditividade das 5 ROIs na última iteração para : (b) Alg_Esparsa. (d) Alg_RRI. Valores obtidos depois de 1920 iterações. Em todos os graficos a região com maior valor de impeditividade corresponde à região 2, na qual está o objeto. Malha de 182 elementos, 156 nós e 32 eletrodos. 


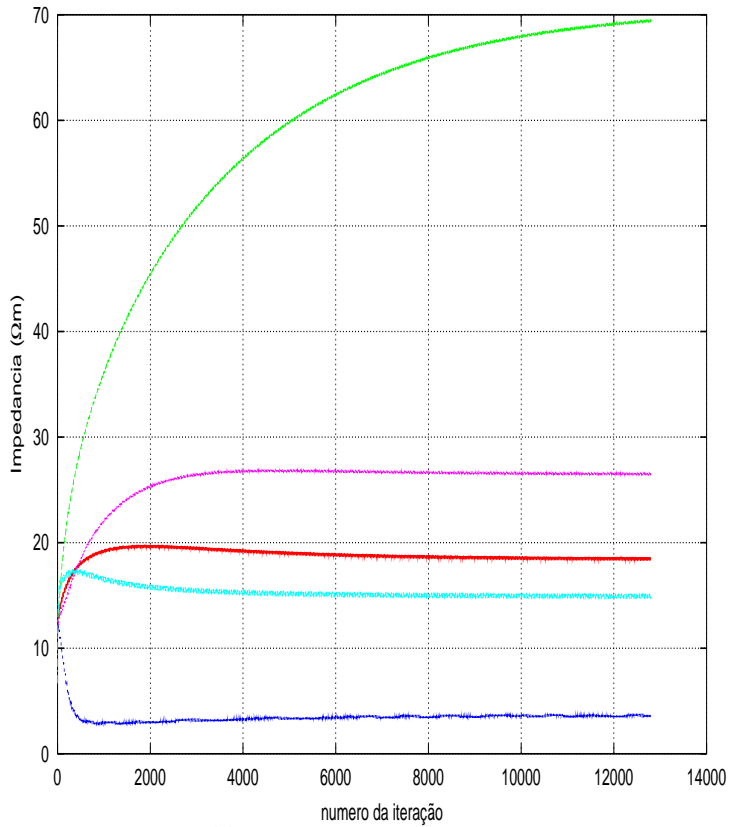

(a)

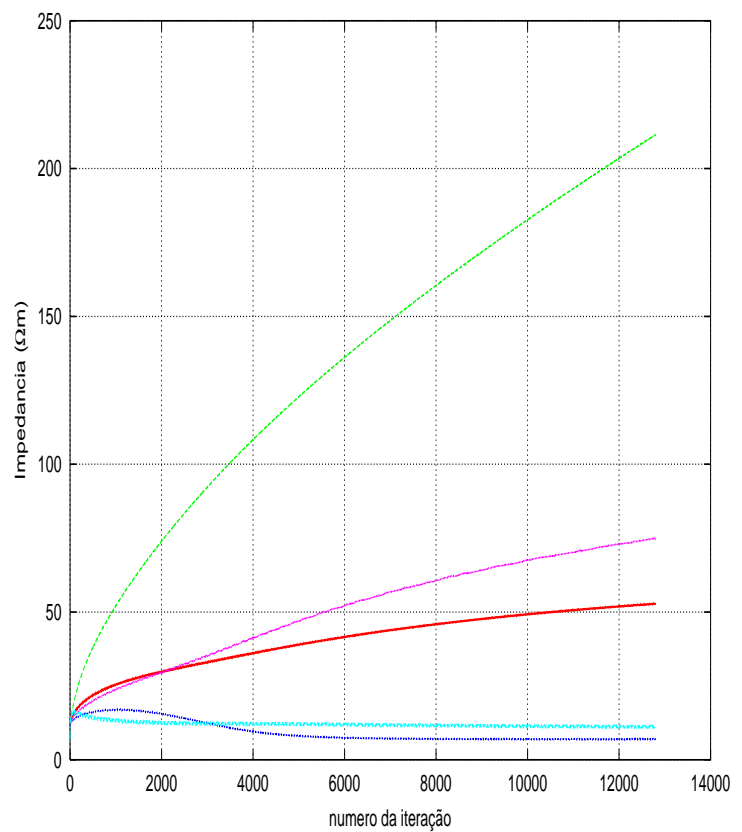

(c)

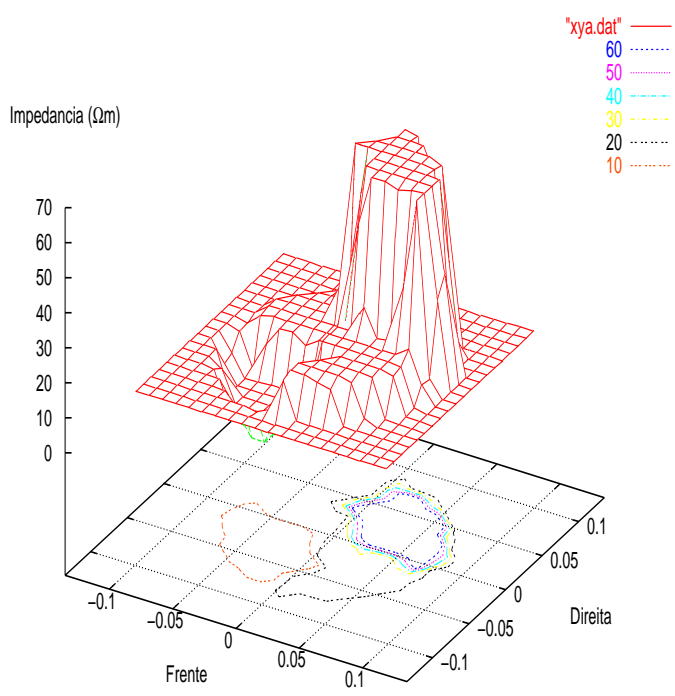

(b)

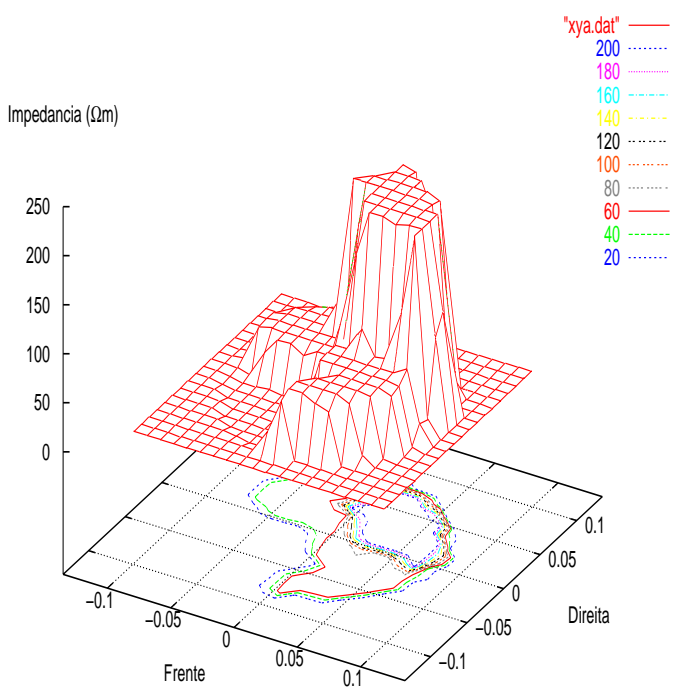

(d)

Figura 7.5: Comparação dos resultados do Agrupamento 1, 5 ROIs. Estimativas de impeditividade no tempo para as 5 ROIs : (a) Alg_Esparsa, (c) Alg_RRI. Representação tridimensional do vetor de impeditividade das 5 ROIs na última iteração para : (b) Alg_Esparsa. (d) Alg_RRI. Valores obtidos depois de 12800 iterações. Em todos os graficos, a região com maior valor de impeditividade corresponde à região 2, na qual está o objeto. Malha de 182 elementos, 156 nós e 32 eletrodos. 
Tabela 7.5: Elementos que constituem as 8 ROIs

\begin{tabular}{|l||c|c|c|}
\hline \hline Região No. & $\hat{\rho}_{(o)}^{(+)}(\Omega m)$ & Elementos que constituem a região & Contém \\
\hline \hline 1 & 11.111 & $1,2,6,7,8,9,45$ & S. salina \\
\hline 2 & 11.111 & $3,4,5,11,12,13,14$ & Objeto \\
\hline 3 & 11.111 & $20,21,22,23,24,29$ & Objeto \\
\hline 4 & 11.111 & $30,31,32,33,37,38,101$ & Objeto \\
\hline 5 & 11.111 & $25,26,27,28,34,35,36$ & S. salina \\
\hline 6 & 11.111 & $10,15,16,17,18,19$ & S. salina \\
\hline 7 & 6.666 & $44,52,60,68,76,84,91$, & S. salina \\
& & $100,108,116,124,132,140,147$ & \\
\hline 8 & 6.666 & o resto de elementos & S. salina \\
\hline \hline$P_{o}$ & $2.0 I_{8}$ & & \\
\hline$R$ & $9.0 \mathrm{e}-02 I_{32}$ & & \\
\hline \hline
\end{tabular}

- Objeto o objeto cilíndrico de vidro que se encontra dentro do phantom.

\subsubsection{Estimativa de Impeditividade em 8 ROIs, Alg_Esparsa}

Foram calculadas as estimativas de impeditividade das ROIs no Alg Esparsa, segundo o agrupamento em 8 ROIs da Figura 7.6. Assim geraram-se as seguintes figuras : Figura 7.7(a) de impeditividade em cada uma das 8 ROIs e Figura 7.7(b) do gráfico tridimensional da impeditividade das 8 ROIs na iteração 1920.

\subsubsection{Estimativa de Impeditividade em 8 ROIs, Alg_RRI}

As estimativas de impeditividade dos elementos da malha renumerada com refinamento iterativo foram obtidos através de padrões de corrente diametrais no phantom.

Geraram-se as seguintes figuras : Figura 7.7(c) de impeditividade em cada uma das 8 ROIs e Figura 7.7(d) do gráfico tridimensional da impedância das 8 ROIs na iteração 1920. 


\section{ROIS}
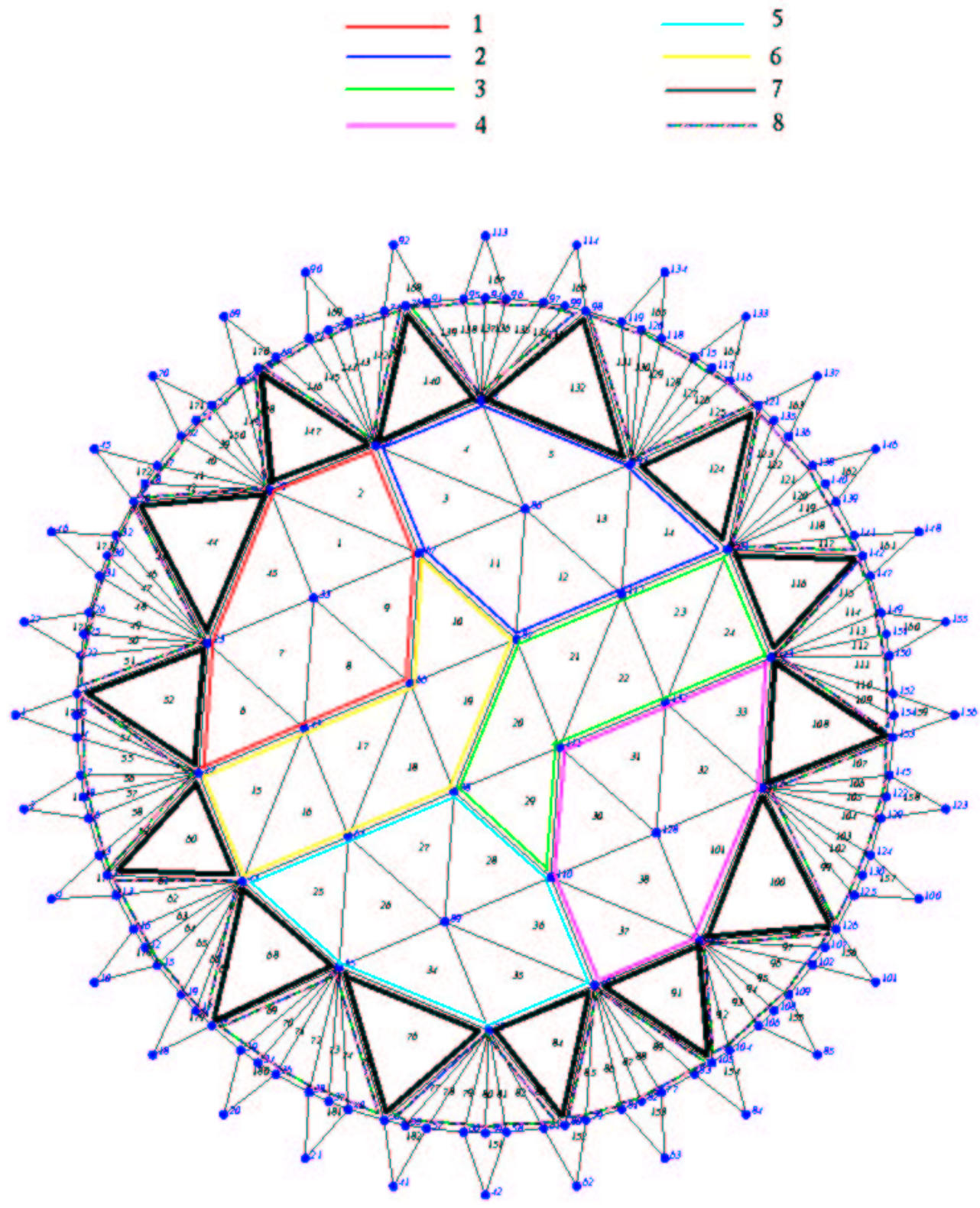

Figura 7.6: Agrupamento 2. 8 regiões de interesse para a malha de 182 elementos, 156 nós e 32 eletrodos. Entre as regiões número 2, 3 e 4 encontra-se o objeto de vidro. 


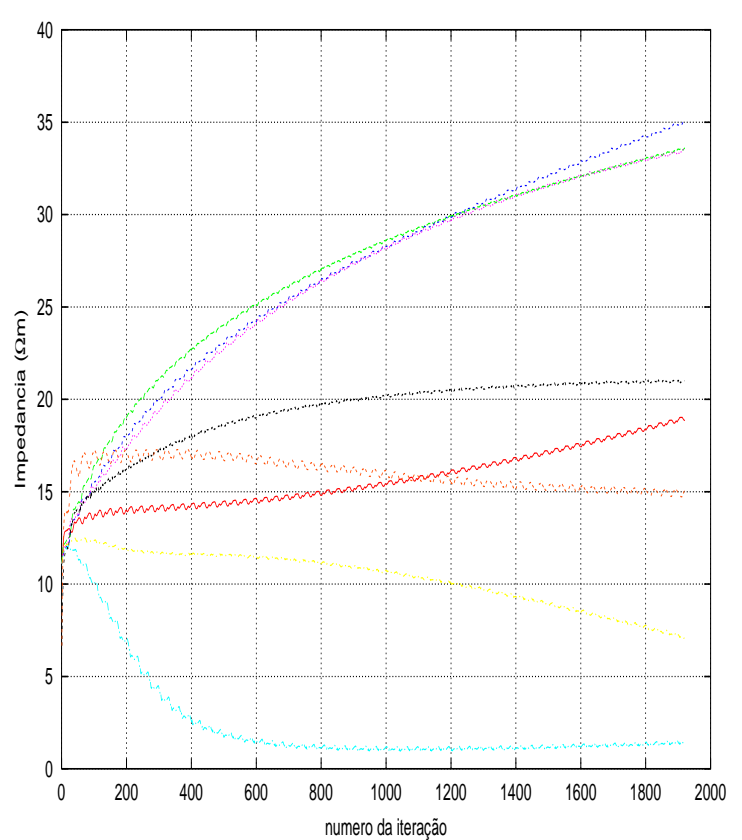

(a)

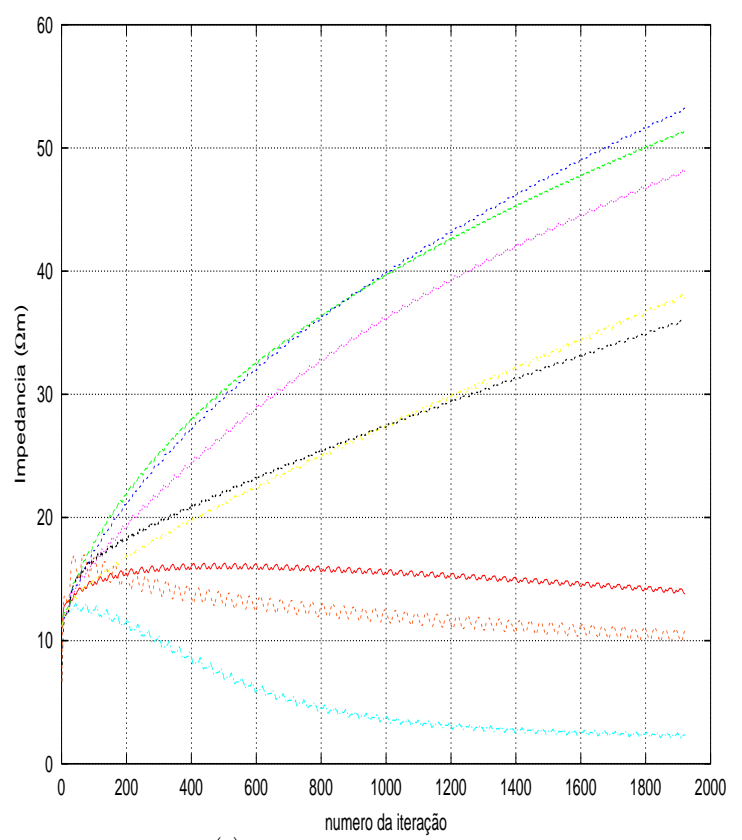

(c)

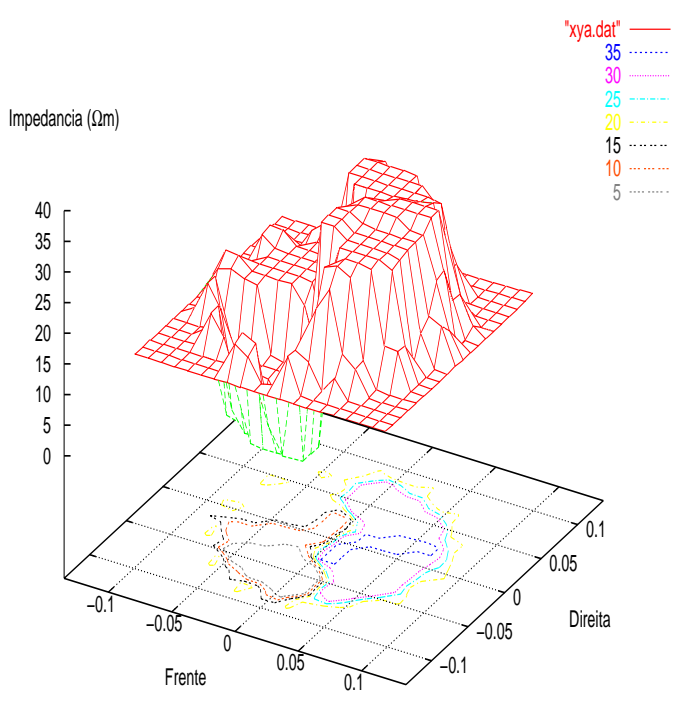

(b)

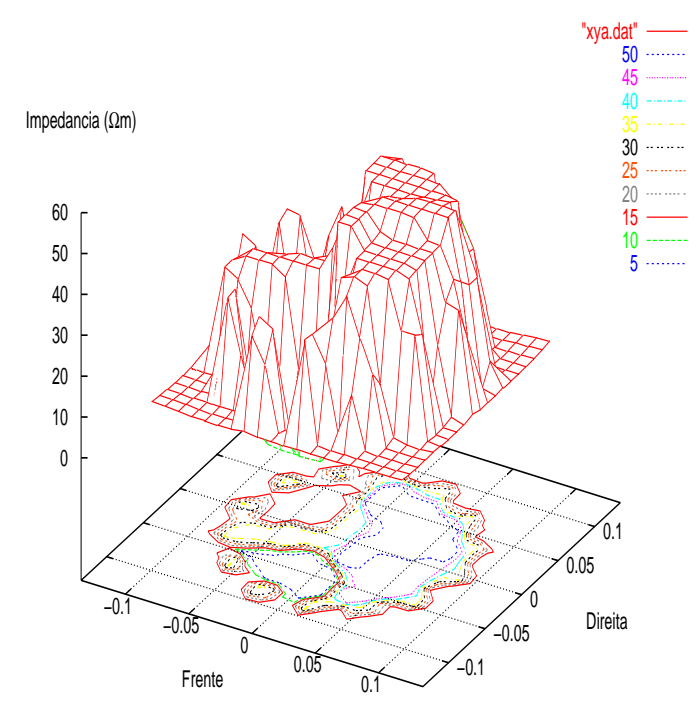

(d)

Figura 7.7: Comparação dos resultados do Agrupamento 2, 8 ROIs. Estimativas de impeditividade no tempo para as 8 ROIs : (a) Alg_Esparsa, (c) Alg_RRI. Representação tridimensional do vetor de impeditividade das 8 ROIs na última iteração para : (b) Alg_Esparsa. (d) Alg_RRI. Valores obtidos depois de 1920 iterações. Em todos os graficos as regiões com maior impeditividade correspondem às regiões 2, 3 e 4 onde se encontra o objeto. Malha de 182 elementos, 156 nós e 32 eletrodos. 


\subsection{Dezesseis Regiões de Interesse (16 ROIs)}

Agruparam-se os 182 elementos da malha de elementos finitos em 16 ROIs, dos quais os últimos 32 elementos da malha são os eletrodos. Na Tabela 7.6 enumeram-se os elementos que constituem cada ROI.

Neste caso quer-se estimar a impeditividade de 16 ROIs para identificar o objeto dentro do phantom o qual se encontra entre as regiões de números 5,9 e 12 .

Tabela 7.6: Elementos que constituem as 16 regiões de interesse para a malha de elementos finitos da Figura 7.8 e parâmetros para o Filtro de Kalman

\begin{tabular}{|l||c|c|c|}
\hline \hline Região No. & $\hat{\rho}_{(o)}^{(+)}(\Omega m)$ & Elementos que constituem a região & Contém \\
\hline \hline 1 & 11.111 & $1,2,45$ & S. salina \\
\hline 2 & 11.111 & $3,4,5$ & S. salina \\
\hline 3 & 11.111 & $6,7,8$ & S. salina \\
\hline 4 & 11.111 & $9,10,11$ & S. salina \\
\hline 5 & 11.111 & $12,13,14$ & Objeto \\
\hline 6 & 11.111 & $15,16,17$ & S. salina \\
\hline 7 & 11.111 & 18,19 & S. salina \\
\hline 8 & 11.111 & 20,21 & S. salina \\
\hline 9 & 11.111 & $22,23,24$ & Objeto \\
\hline 10 & 11.111 & $25,26,27$ & S. salina \\
\hline 11 & 11.111 & $28,29,30$ & S. salina \\
\hline 12 & 11.111 & $31,32,33$ & Objeto \\
\hline 13 & 11.111 & $34,35,36$ & S. salina \\
\hline 14 & 11.111 & $37,38,101$ & S. salina \\
\hline 15 & 6.666 & $44,52,60,68,76,84,91$, & S. salina \\
\hline 16 & & $100,108,116,124,132,140,147$ & \\
\hline \hline$P_{o}$ & 6.666 & o resto de elementos & S. salina \\
\hline$R$ & $9.0 \mathrm{e}-02 I_{32}$ & & \\
\hline \hline
\end{tabular}

Na tabela anterior, indicam-se por :

- $\hat{\rho}_{(o)}^{(+)}$a condição inicial do vetor de impedâncias para as regiões;

- S. salina a solução salina, com estimativa de impedância $17 \Omega m$;

- Objeto o objeto cilíndrico de vidro que se encontra dentro do phantom. 
16 ROIS

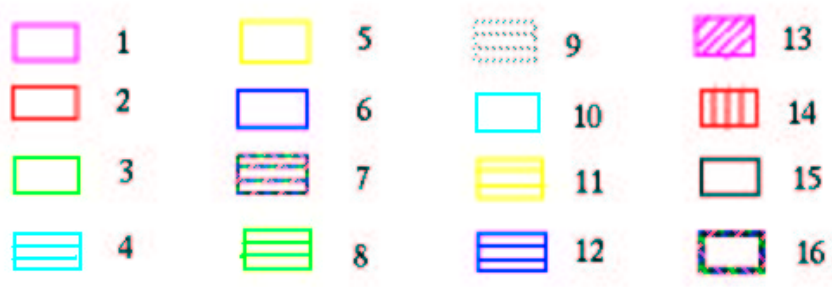

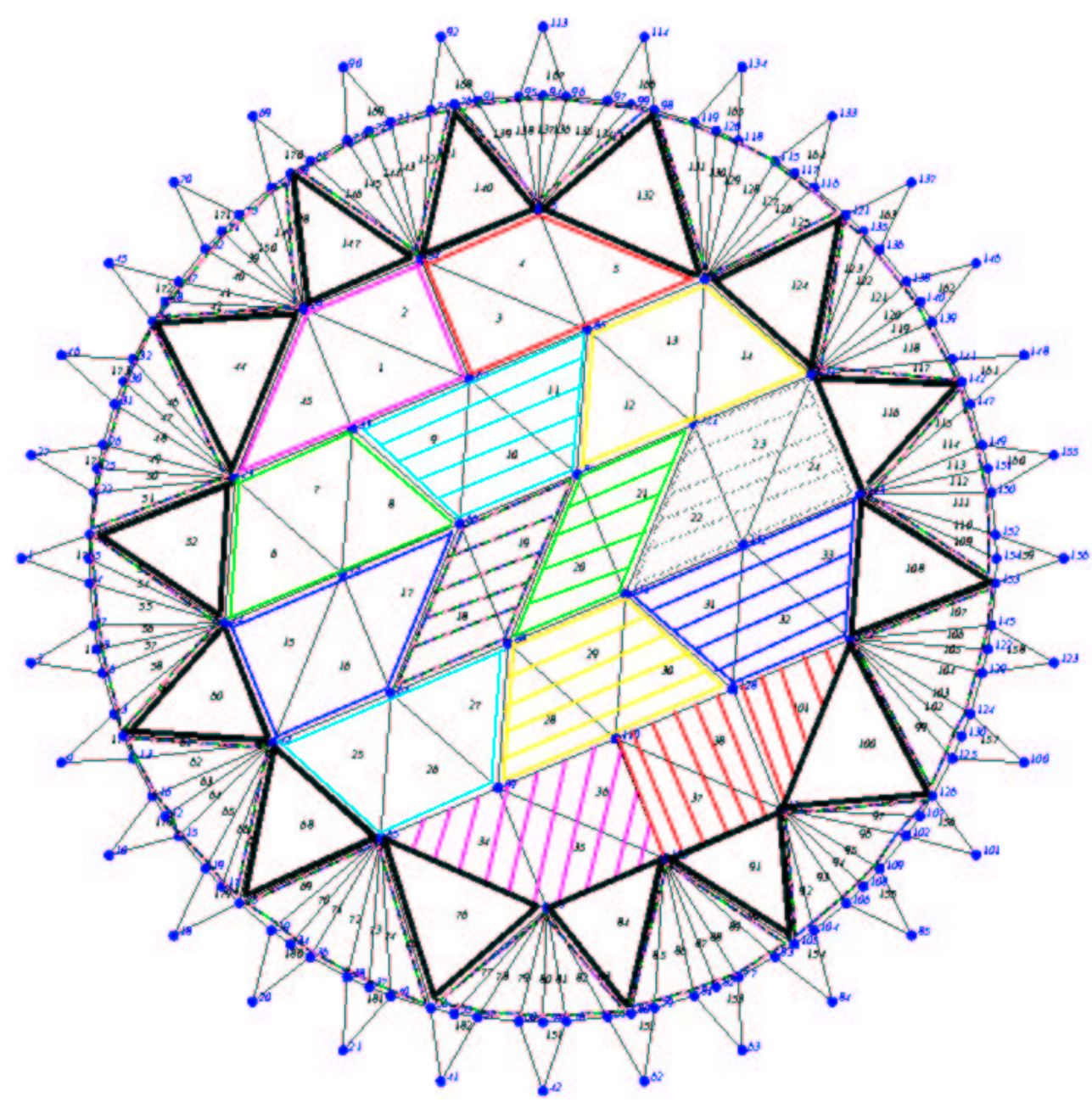

Figura 7.8: Agrupamento 3. 16 regiões de interesse para a malha de 182 elementos, 156 nós e 32 eletrodos. Entre as regiões número 5, 9 e 12 encontra-se o objeto de vidro. 


\subsubsection{Estimativa de Impeditividade em 16 ROIs, Alg_Esparsa}

Foram calculadas as estimativas de impeditividade de ROIs usando Alg Esparsa.

Geraram-se as seguintes figuras : Figura 7.9(a) de impeditividade em cada ROI e a Figura 7.9(b) do gráfico tridimensional da impedância das 16 ROIs na iteração 1920.

\subsubsection{Estimativa de Impeditividade em 16 ROIs, Alg_RRI}

Foram calculadas as estimativas de impeditividade de ROIs usando Alg_RRI, usando dados experimentais obtidos aplicando o padrões de corrente diametrais.

Geraram-se as seguintes figuras : Figura 7.9(c) de impeditividade em cada uma ROI e a Figura 7.9(d) do gráfico tridimensional da impeditividade das 16 ROIs na iteração 1920. 


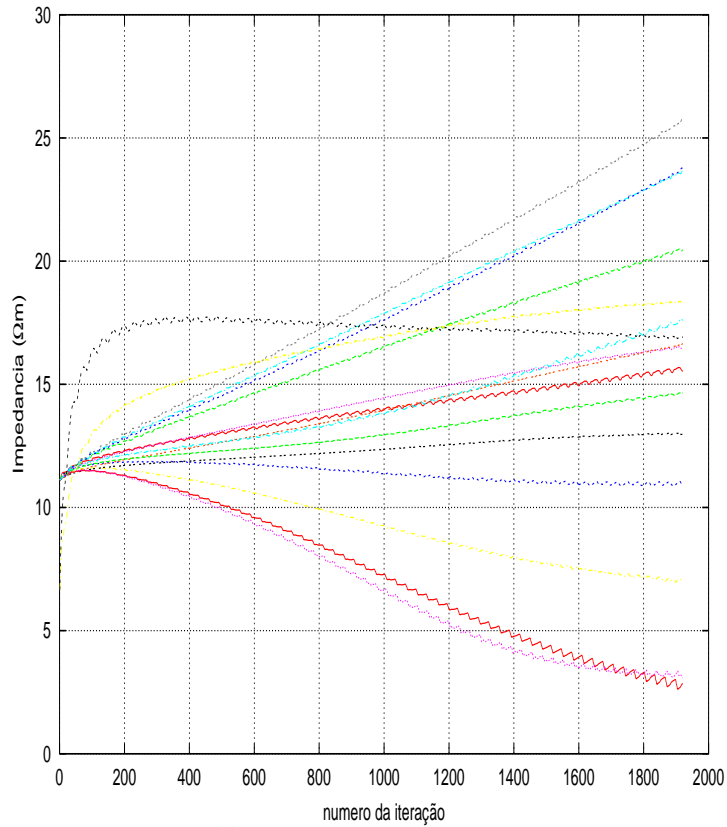

(a)

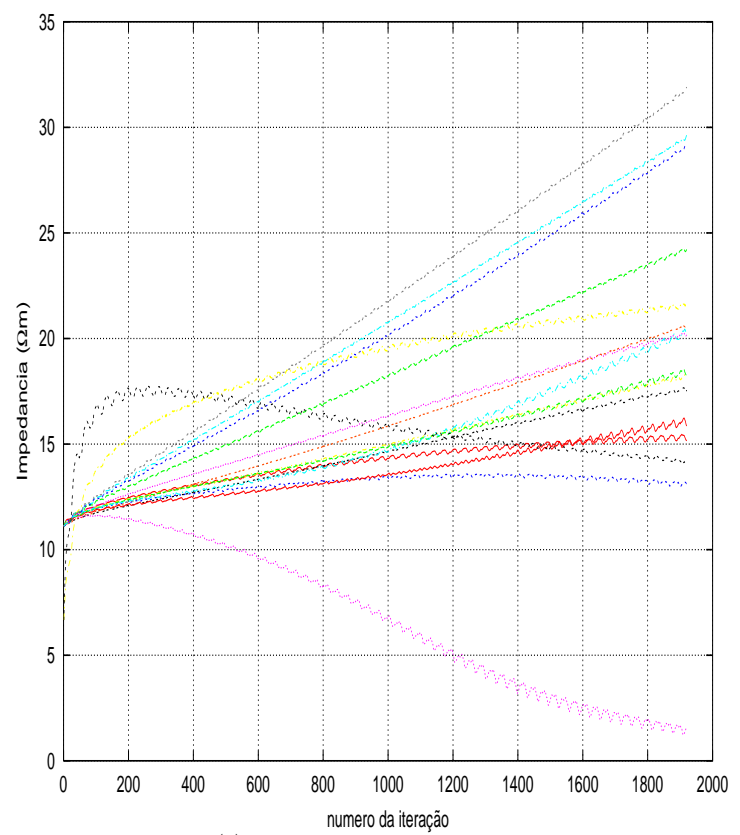

(c)

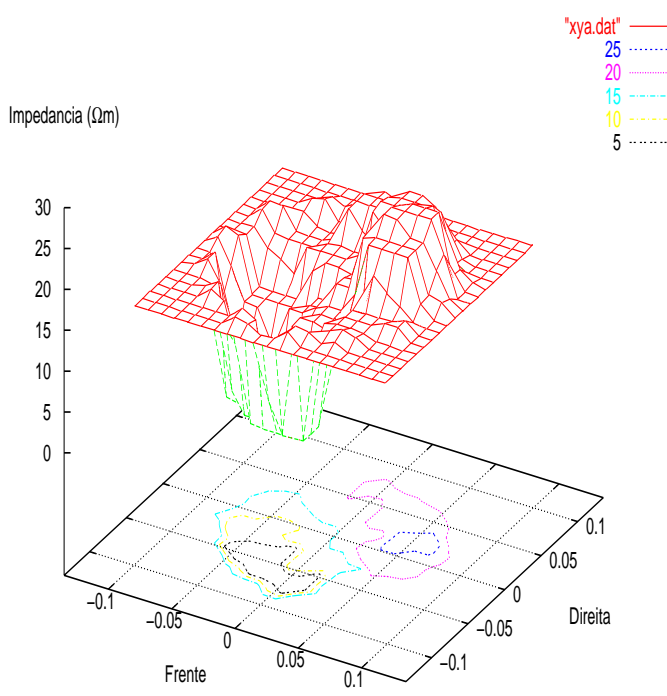

(b)

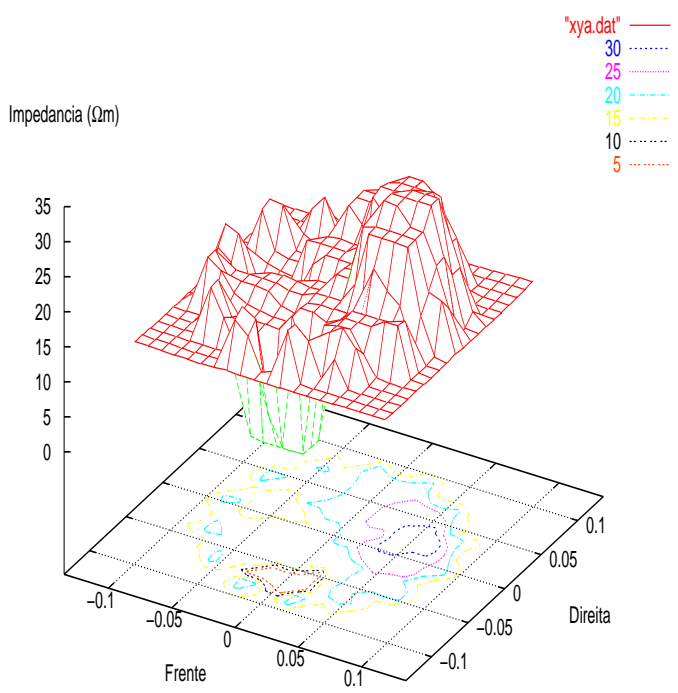

(d)

Figura 7.9: Comparação dos resultados do Agrupamento 3, 16 ROIs. Estimativas de impeditividade no tempo para as 16 ROIs : (a) Alg_Esparsa, (c) Alg_RRI. Representação tridimensional do vetor de impeditividade das 16 ROIs na última iteração para : (b) Alg_Esparsa. (d) Alg_RRI. Valores obtidos depois de 1920 iterações. Em todos os gráficos as regiões com maior impeditividade correspondem às regiões 5, 9 e 12 onde se encontra o objeto. Malha de 182 elementos, 156 nós e 32 eletrodos. 


\subsection{Comparação dos Resultados para 5, 8 e 16 ROIs}

Com o objetivo de facilitar a comparação dos diferentes resultados obtidos com as duas versões Alg_Esparsa e Alg_RRI, nos três tipos de agrupamentos 5, 8 ou 16 ROIs, elaborou-se a Tabela 7.7. Nela mostram-se os valores das impeditividade estimadas na iteração 1920. Lembre-se que os parâmetros do Filtro Estendido de Kalman utilizados na estimação da impeditividade para cada tipo de agrupamento (5, 8 ou 16 ROIs) são os mesmos. O algoritmo desenvolvido para TIE foi avaliado com dados experimentais para identificar a(s) região(ões) que contém o objeto de vidro.

Em todos os agrupamentos as estimativas da impeditividade são melhores na versão 2 , da Alg_RRI. Os valores de impeditividade apresentados na Tabela 7.7 têm unidades $(\Omega m)$.

onde

- $\hat{\rho}_{(o)}^{(+)}$: condição inicial do vetor de impeditividade para as regiões;

- $\hat{\rho}^{(+)}$: estimativa do vetor de impeditividade para as regiões; 
Tabela 7.7: Resultados de impeditividade nas ROIs obtidos pelas duas versões. Alg_Esparsa e Alg_RRI

\begin{tabular}{|c|c|c|c|c|c|c|}
\hline Agrupamento & No.ROIs & Região No. & $\begin{array}{l}\hat{\rho}_{(o)}^{(+)} \\
(\Omega m)\end{array}$ & $\begin{array}{l}\text { Versão } 1 \\
\text { Alg_Esparsa } \\
\hat{\rho}^{(+)}(\Omega m)\end{array}$ & $\begin{array}{l}\text { Versão } 2 \\
\text { Alg_RRI } \\
\hat{\rho}^{(+)}(\Omega m)\end{array}$ & Contém \\
\hline \multirow[t]{5}{*}{1} & \multirow[t]{5}{*}{5} & 1 & 11.11 & 19.51 & 29.25 & S. salina \\
\hline & & 2 & 11.11 & 44.95 & 72.47 & Objeto \\
\hline & & 3 & 11.11 & 2.93 & 15.68 & S. salina \\
\hline & & 4 & 11.11 & 25.16 & 29.06 & S. salina \\
\hline & & 5 & 6.66 & 15.94 & 13.00 & S. salina \\
\hline \multirow[t]{8}{*}{2} & \multirow[t]{8}{*}{8} & 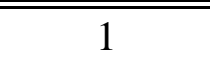 & 11.11 & 18.86 & 13.81 & S. salina \\
\hline & & 2 & 11.11 & 33.57 & 51.40 & Objeto \\
\hline & & 3 & 11.11 & 35.10 & 53.22 & Objeto \\
\hline & & 4 & 11.11 & 33.47 & 48.11 & Objeto \\
\hline & & 5 & 11.11 & 1.38 & 2.34 & S. salina \\
\hline & & 6 & 11.11 & 7.09 & 37.85 & S. salina \\
\hline & & 7 & 6.66 & 21.00 & 35.97 & S. salina \\
\hline & & 8 & 6.66 & 15.01 & 10.74 & S. salina \\
\hline \multirow[t]{16}{*}{3} & \multirow[t]{16}{*}{$\overline{16}$} & 1 & 11.11 & 15.54 & 15.17 & S. salina \\
\hline & & 2 & 11.11 & 20.45 & 24.20 & S. salina \\
\hline & & 3 & 11.11 & 10.90 & 13.12 & S. salina \\
\hline & & 4 & 11.11 & 16.49 & 20.16 & S. salina \\
\hline & & 5 & 11.11 & 23.67 & 29.61 & Objeto \\
\hline & & 6 & 11.11 & 6.98 & 18.15 & S. salina \\
\hline & & 7 & 11.11 & 12.98 & 17.53 & S. salina \\
\hline & & 8 & 11.11 & 16.64 & 20.60 & S. salina \\
\hline & & 9 & 11.11 & 25.75 & 31.87 & Objeto \\
\hline & & 10 & 11.11 & 2.85 & 15.87 & S. salina \\
\hline & & 11 & 11.11 & 14.64 & 18.31 & S. salina \\
\hline & & 12 & 11.11 & 23.76 & 29.13 & Objeto \\
\hline & & 13 & 11.11 & 3.09 & 1.59 & S. salina \\
\hline & & 14 & 11.11 & 17.60 & 20.21 & S. salina \\
\hline & & 15 & 6.66 & 18.35 & 21.51 & S. salina \\
\hline & & 16 & 6.66 & 16.91 & 14.24 & S. salina \\
\hline
\end{tabular}


- Alg_Esparsa : Algoritmo inicial com matriz de condutividade esparsa;

- Alg_RRI : Algoritmo com malha renumerada e refinamento iterativo;

- S. salina : solução salina, estimativa de impedância $17 \Omega m$;

- Objeto : objeto cilíndrico de vidro que se encontra dentro do phantom

Em 5 ROIs, as estimativas de impedância na cor vermelha identificam a região 2 onde se encontra o objeto de vidro;

Em 8 ROIs, as estimativas de impedância na cor azul identificam as ROIs 2, 3 e 4 onde se encontra o objeto de vidro;

Em 16 ROIs,as estimativas de impedância na cor magenta identificam as ROIs 5, 9 e 12 onde se encontra o objeto de vidro;

Na Figura 7.10 são reproduzidos os gráficos tridimensionais do vetor de impeditividade para 5, 8 ou 16 ROIs para Alg_Esparsa e Alg_RRI, com o objetivo de facilitar a comparação dos resultados obtidos.

Verifica-se a convergência do Filtro de Kalman através do resíduo de medida $\left(r_{v}\right)$, apresentado na equação $7.1[13]$ :

$$
r_{v}=\left\{1 / n f \sum_{j=1}^{n f}\left[v_{k}^{(n f)}(j)-h_{k}^{(n f)}(j)\right]\right\} / \rho_{v}, j=1,2, . . n f
$$

onde

$r_{v}:$ resíduo normalizado;

$n f:$ número de eletrodos;

$v_{k}^{(n f)}$ : medida real, dados experimentais das voltagens nos eletrodos;

$h_{k}^{(n f)}$ : valor calculado das voltagens nos eletrodos;

$\rho_{v}$ : desvio padrão dos ruídos na medida $\rho_{v}=0.3$, para 5,8 ou 16 ROIs.

A convergência é verificada visto que $E\left[r_{v}\right]=0$ durante o processo de estimação e $-3 \rho_{v} \leq$ $r_{v} \leq 3 \rho_{v}$. Com o objetivo de facilitar a comparação dos resultados obtidos, na Figura 7.11 apresenta-se o resíduo normalizado para 5, 8 ou 16 ROIs usando as duas versões do algoritmo.

\subsection{Avaliação dos Resultados}

Analisa-se nesta seção a influência das perturbações na inversa da matriz de condutividade $\delta Y^{-1}$ e da matriz de sensibilidade $\delta H$, devido a sua importância nas equações que regem a solução do problema de TIE. Avaliadas estas perturbações compara-se o seu comportamento quando se obtêm resultados com Alg_Esparsa e Alg_RRI. 


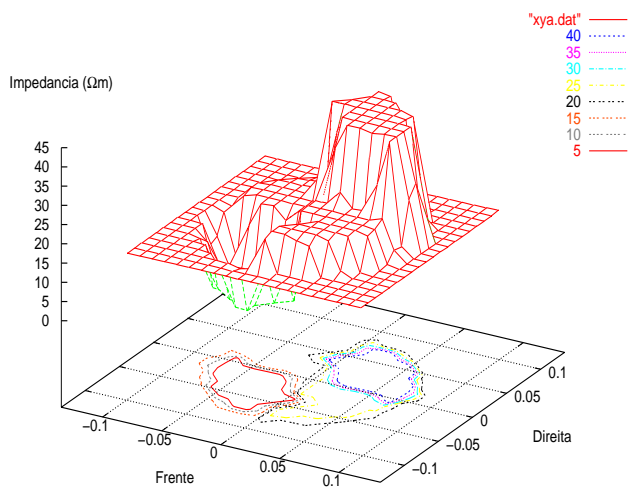

(a)

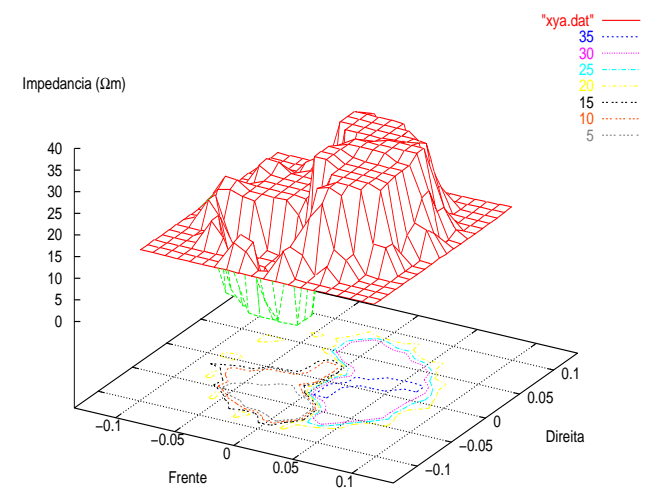

(c)

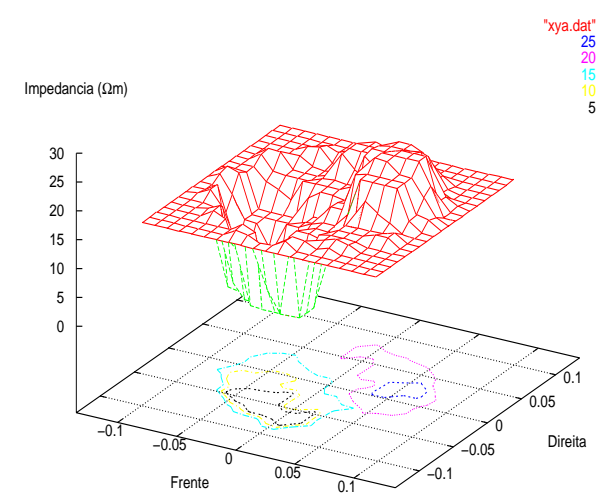

(e)

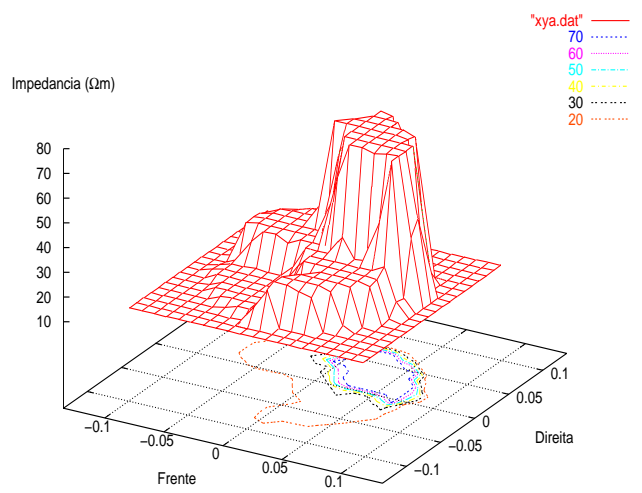

(b)

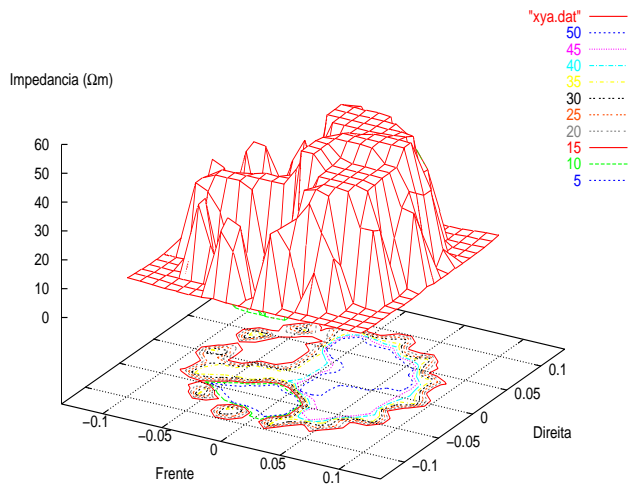

(d)

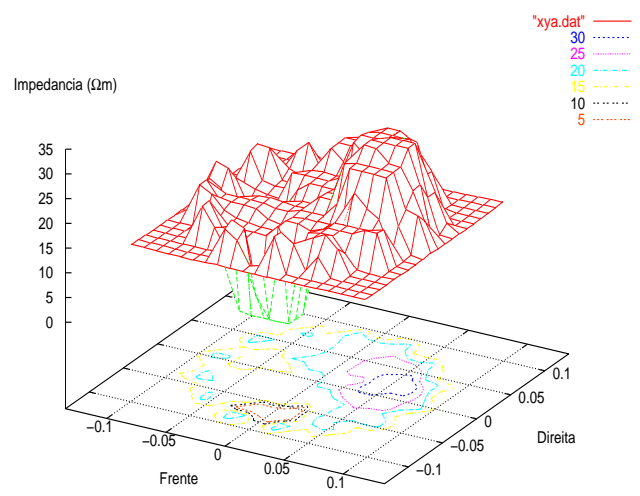

(f)

Figura 7.10: Comparação dos resultados da representação tridimensional do vetor de impeditividade para Alg_Esparsa de : (a) 5 ROIs, (c) 8 ROIs, (e) 16 ROIs; e Alg_RRI de : (b) 5 ROIs, (d) 8 ROIs, (f) 16 ROIs. Malha com 182 elementos, 156 nós e 32 eletrodos. 


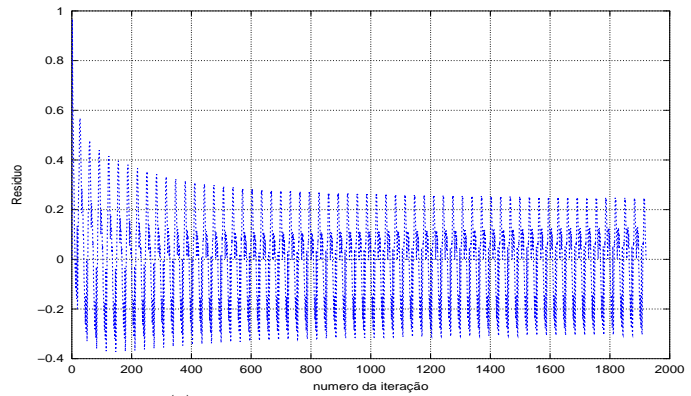

(a)

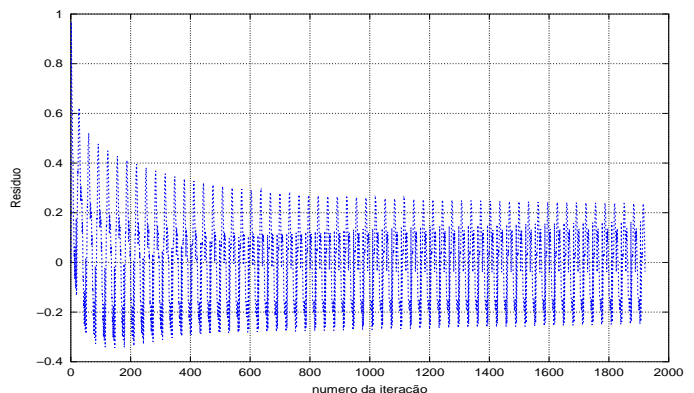

(c)

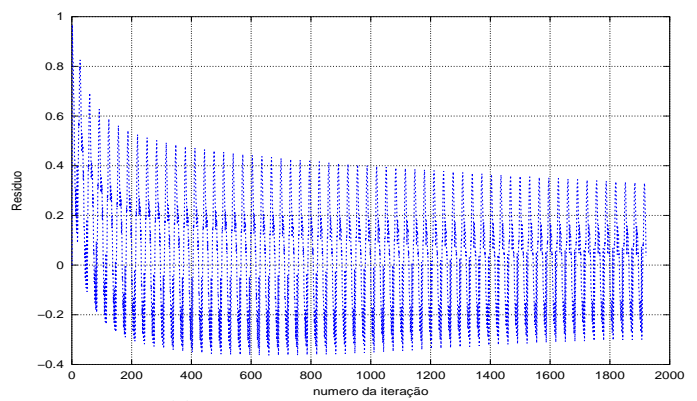

(e)

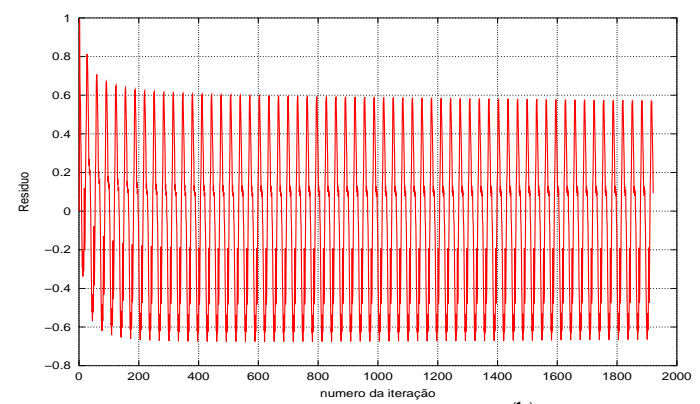

(b)

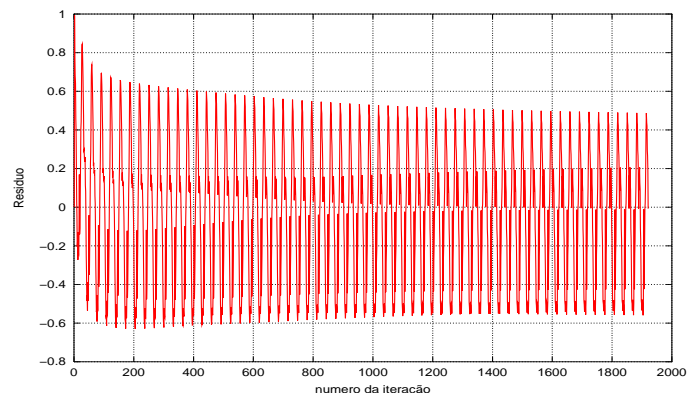

(d)

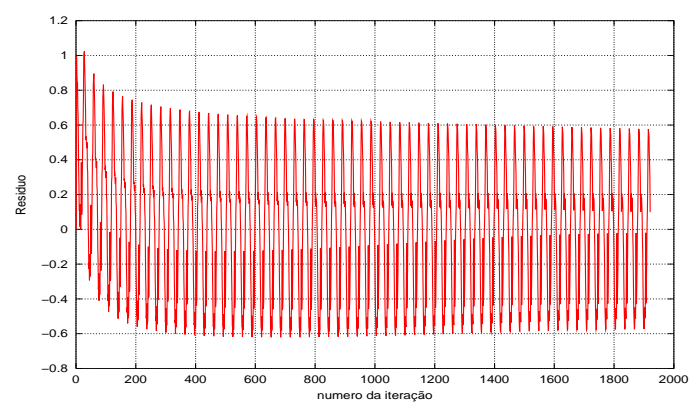

(f)

Figura 7.11: Comparação dos resultados do resíduo normalizado para Alg_Esparsa de (a) 5 ROIs, (c) 8 ROIs, (e) 16 ROIs e Alg_RRI de (b) 5 ROIs, (d) 8 ROIs, (f) 16 ROIs. Malha com 182 elementos, 156 nós e 32 eletrodos. Consideração de convergência verificada para as duas versões. 
Segundo a teoria da perturbação, Watkins [37], pode-se explicar o comportamento das perturbações na solução de um sistema linear a partir do teorema (2.3.17), descrito a seguir.

Se $A$ é não singular, e

$$
\frac{\|\delta A\|}{\|A\|}<\frac{1}{k(A)}
$$

então $A+\delta A$ não é singular

onde

$\delta A$ : perturbações da matriz $A$,

$\|A\|:$ norma de $A$,

$\|\delta A\|$ : norma de $\delta A$,

$k(A)$ : número de condição da matriz $A$, definido em [29] como o quociente entre o maior e o menor valor singular obtidos na decomposição de valores singulares.

Substituindo $A$ pelo inversa da matriz de condutividade $Y^{-1}$ na desigualdade 7.2 , tem-se :

$$
\frac{\left\|\delta Y^{-1}\right\|}{\left\|Y^{-1}\right\|} \leq \frac{1}{k\left(Y^{-1}\right)}
$$

onde

$\delta Y^{-1}$ : perturbações na inversa da matriz de condutividade $Y$,

$\left\|Y^{-1}\right\|:$ norma de $Y^{-1}$,

$\left\|\delta Y^{-1}\right\|:$ norma de $\delta Y^{-1}$,

$k\left(Y^{-1}\right)$ : número de condição da matriz $Y^{-1}$.

A partir da desigualdade 7.3 pode-se determinar o limite superior da perturbação na inversa da matriz de condutividade para os dois algoritmos,Alg_Esparsa e Alg_RRI. Considera-se um phantom com uma distribuição de impedâncias conhecida, aqui a matriz de condutividade $Y$ é conhecida.

- as impedâncias das regiões de interesse onde se encontra o objeto são iguais a $60 \Omega m$;

- impedâncias para o resto das regiões de interesse são iguais a $17 \Omega m$.

Foi desenvolvido um algoritmo em C para efetuar os cálculos da equação 7.3 e a Tabela 7.8 apresentam-se os resultados obtidos das perturbações em cada versão.

Na Tabela 7.8 observa-se que a relação 7.3 é verificada em ambos os casos. Da tabela pode-se observar ainda que o quociente $\left\|\delta Y^{-1}\right\| /\left\|Y^{-1}\right\|$ é dez vezes menor para Alg_RRI. Isto explica a melhor resolução espacial e a melhor estabilidade do algoritmo do Filtro de Kalman.

O termo $\left\|Y . Y^{-1}-I\right\|$ na Tabela 7.8 é o maior valor singular de $\left(Y . Y^{-1}-I\right)$, isto mostra a ordem de grandeza das perturbações na matriz identidade, sendo 3 vezes menor para Alg_RRI. 
Tabela 7.8: Limite superior na perturbação na inversa da matriz de condutividade

\begin{tabular}{|l||l|l|}
\hline \hline Relação & $\begin{array}{l}\text { Versão 1. } \\
\text { Alg_Esparsa }\end{array}$ & $\begin{array}{l}\text { Versão } 2 . \\
\text { Alg_RRI }\end{array}$ \\
\hline \hline$\left\|Y . Y^{-1}-I\right\|$ & $1.4228635630830766 \mathrm{e}-14$ & $5.0604683238134138 \mathrm{e}-15$ \\
\hline$k\left(Y^{-1}\right)$ & $9.2643869830663665 \mathrm{e}+04$ & $9.2643869830654483 \mathrm{e}+04$ \\
\hline $1 / k\left(Y^{-1}\right)$ & $1.0794022333348340 \mathrm{e}-05$ & $1.0794022333349409 \mathrm{e}-05$ \\
\hline$\left\|\delta Y^{-1}\right\|$ & $4.3939040064399976 \mathrm{e}-09$ & $4.2803961782601861 \mathrm{e}-10$ \\
\hline$\left\|\delta Y^{-1}\right\| /\left\|Y^{-1}\right\|$ & $4.7427897976103793 \mathrm{e}-14$ & $4.6202691943723166 \mathrm{e}-15$ \\
\hline \hline
\end{tabular}

A inversa da matriz de condutividade e a sua perturbação têm efeitos sobre a matriz de sensibilidade e sua perturbação. A matriz de sensibilidade $H$ tem grande influência no comportamento do Filtro de Kalman e nas imagens.

Para obter a perturbação $\delta H$, parte-se da equação da matriz de sensibilidade $H$ :

$$
H=-Y^{-1} \frac{\partial Y}{\partial \sigma} Y^{-1} c_{j} .
$$

Somando a perturbação à matriz de sensibilidade resulta :

$$
H+\delta H=-\left(Y^{-1}+\delta Y^{-1}\right)\left[\frac{\partial Y}{\partial \sigma}+\delta\left(\frac{\partial Y}{\partial \sigma}\right)\right]\left(Y^{-1}+\delta Y^{-1}\right) c_{j} .
$$

Desprezando termos de ordem 2 a equação 7.5 torna-se

$$
H+\delta H=\left[-Y^{-1} \frac{\partial Y}{\partial \sigma} Y^{-1}\right] c_{j}+\left[-Y^{-1} \frac{\partial Y}{\partial \sigma} \delta Y^{-1}\right] c_{j}+\left[-\delta Y^{-1} \frac{\partial Y}{\partial \sigma} Y^{-1}\right] c_{j}
$$

Da equação 7.6 e da equação 7.4 obtém-se a perturbação da matriz de sensibilidade $\delta H$ :

$$
\delta H=-\left[Y^{-1} \frac{\partial Y}{\partial \sigma} \delta Y^{-1}+\delta Y^{-1} \frac{\partial Y}{\partial \sigma} Y^{-1}\right] c_{j} .
$$

A equação 7.7 indica que a perturbação na matriz de sensibilidade é proporcional à perturbação na inversa da matriz de condutividade. 


\section{Capítulo 8}

\section{CONCLUSÕES E SUGESTÕES PARA TRABALHOS FUTUROS}

Uma melhora de precisão na impeditividade e uma melhora na velocidade de convergência foram obtidas através da implementação de operações algebricas matriciais em representação compacta e rotinas de melhoramento iterativo da solução de sistemas lineares.

Segundo os resultados obtidos em todos os três casos de regiões de interesse 5, 8 ou 16, as estimativas de impeditividade são melhores quando a malha é renumerada e representada em forma compacta, conforme a Tabela 7.7. Isto decorre da diminução da perturbação na inversa da matriz de condutividade e na matriz de sensibilidade, as quais tem influência na estabilidade do algoritmo de Filtro Estendido de Kalman, conforme a Tabela 7.8 e a equação 7.7.

A renumeração a malha de elementos finitos, leva a uma matriz de condutividade de banda limitada e posteriormente a uma matriz de condutividade em forma compacta. O tamanho da matriz de condutividade passa de 156x156 para 156x77 na malha de 182 elementos. Operações matriciais com matrizes em representação compacta diminui erros numéricos e o tempo computacional para o cálculo da inversa da matriz de condutividade em $25 \%$.

Os parâmetros $P_{o}^{(+)}, Q$ e $R$ do Filtro de Kalman têm influência sobre a estabilidade do algoritmo. Utilizar $P_{o}^{(+)}$para definir $Q$ [6] partindo de $P_{o}$ diagonal, permite obter de forma mais simples e mais eficiente as matrizes de covariância. Este procedimento leva a melhores resultados de estimativas de impedância em todos os casos.

Os estudos aqui realizados restringem-se a aplicação do padrão de corrente diametral com modelo completo de eletrodos. O desempenho do Filtro de Kalman depende destes fatores.

Algumas sugestões para futuros trabalhos incluem :

- aplicação de padrões de corrente ótimos para identificar melhor regiões específicas; 
- melhorar as bancadas existentes com diferentes materiais que tenham impedâncias similares ás do corpo humano;

- diminuir o tamanho da matriz de condutividade através de condensação estática;

- utilizar formas equivalentes do Filtro de Kalman, visando evitar inversões de matrizes;

- aperfeiçoar as matrizes de covariâncias de erro estimação e do ruído no estado para cada aplicação particular, eventualmente de forma adaptativa;

- o observador de estado depende da qualidade do conjunto de medidas de voltagem. Assim, propõe-se desenvolver um método que permita detectar erros grosseiros nas medidas;

- implementar uma representação compacta para a matriz de sensibilidade. 


\section{Referências Bibliográficas}

[1] AMATO, A. 2002. Diagnósticos medicos, ultrassonografia. Disponível em : <http://

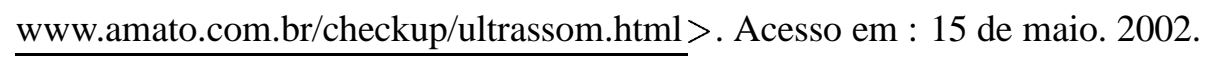

[2] AMATO, M.B.P. et al. Effect of a Protective-Ventilation Strategy on Mortality in the Acute Respiratory Distress Syndrome. Journal of Medicine, New England, 338(6), p.347-354, 1998.

[3] AMATO, M.B.P. Novas Estratégias em Ventilação Artificial: Diagnóstico e Prevenção do Barotrauma/Biotrauma Através da Tomografia de Impedância Elétrica. São Paulo. Março 2001. Projeto Temático - FAPESP.

[4] BARBER, D.C.; BROWN, B.H. Applied Potencial Tomography. The Institute of Physics J. Phys. E: Sci. Instrum., v.17, p.723-733, 1984.

[5] BARBER, D.C. Quantification in Impedance Imaging. Institute of Physical Sciences in Medicine Clin. Phys. Physiol. Meas., v.11, Suppl. A, p.45-56, 1990.

[6] BROGAN, W.L. Modern Control Theory. Second Edition, Prentice-Hall, Inc. Englewood Cliffs, New Jersey, 1985. 509p.

[7] BROWN, B.H.; BARBER, D.C. Applied Potential Tomography - A New in Vivo Medical Imaging Technique. Clin. Phys. Physiol. Meas., v.4, 1982.

[8] BROWN, R.G. Random Signal Analysis and Kalman Filtering. New York, John Wiley and Sons, 1983. 347p.

[9] CAVAlCANTI, L.P. Imagens Medicas. Disponível em : <http:// www.raioxis.com>. Acesso em : 3 de Abril. 2002. 
[10] CENTRO DE TRATAMENTO E PESQUISAS, HOSPITAL DO CÂNCER - A.C. CAMARGO. Departamento de Imagen. Disponível em : <http:// www.hcanc.org.br>. Acesso em : 19 de maio. 2002.

[11] CIBER HABITAT, CIUDAD DE LA INFORMATICA - HOSPITAL. Resonancia Magnética. Disponível em : <http:// www.ciberhabitat.gob.mx/hospital/rm/00.html >. Acesso em : 5 de Abril. 2002.

[12] DEPARTAMENTO DE FÍSICA DA UNIVERSIDADE FEDERAL DO CEARÁ. Fortaleza. Aplicações Modernas dos Raios X na Medicina. Disponível em : <http://

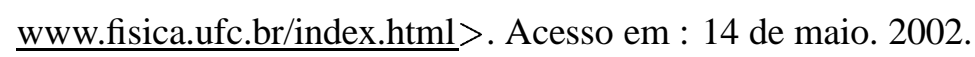

[13] FLEURY, A.T. Estimadores de Estado de Sistemas Dinâmicos Baseados no Conceito de Dualidade. 1985. 161p. Tese (Doutorado) - Escola Politénica, Universidade de São Paulo. São Paulo.

[14] FLEURY, A.T. Filtro de Kalman Aplicado à Navegação Aérea. São Paulo: Escola Politécnica da Universidade de São Paulo, 1999. Notas de Aula da Disciplina de PósGraduação PMC-5759.

[15] FUKS, L.P. et al. Detection and Imaging of Electrical Conductivity and Permittivity at Low Frequency. IEEE Transactions on Biomedical Engineering, v.38, n.11, p.1106-1110, November 1991.

[16] GIBBS, N.E.; POOLE, W.G.; STOCKMEYER, P.K. An Algorithm for Reducing the Bandwidth and Profile of a Sparse Matrix. SIAM J. Numer. Anal, v.13, n.2, p.236-250, April 1976.

[17] GUARDO, R. et al. An Experimental Study in Electrical Impedance Tomography Using Backprojection Reconstruction. IEEE Transactions on Biomedical Engineering, v.38, n.7, p.617-627, July 1991.

[18] HOROWITZ, P.; HILL, W. The Art of Electronics. Second edition. Cambridge: Cambridge University Press, 1989. 1125p.

[19] HUA, P. et al. Using Compound Electrodes in Electrical Impedance Tomography. IEEE Transaction on Biomedical Engineering, v.40, n.1, p.29-34, January 1993. 
[20] HUA, P. et al. Finite Element Modeling of Electrode-Skin Contact Impedance in Electrical Impedance Tomography. IEEE Transaction on Biomedical Engineering, v.40, n.4, p.335343, April 1993.

[21] ISAACSON, D.; CHENEY, M.; NEWELL, J.C. Comments on Reconstruction Algorithms. Institute of Physical Sciences in Medicine Clin. Phys. Physiol. Meas., v.13, Suppl. A, p.83-89, 1992.

[22] KALMAN, R.E. A New Approach to Linear Filtering and Prediction Theory. Trans. ASME, J. of Basic. Engineering, Ser. D, 82, p.35-45, March 1960.

[23] KALMAN, R.E. A Retrospective After Twenty Years : From the Pure to the Applied. In : Chiu, C.L. Proc. AGU, Chapman Conference on Application of Kalman Filter to Hidrology, Hydraulics and Water Resources. Dep. of Civil Eng., University of Pittsburgh , Pittsburgh, p.31-34, 1978.

[24] KEITHLEY METRABYTE. DAS-1600/1400 Series, User's Guide. Keithley Metrabyte Division. Keithley Instruments, Inc. Revision B - Part Number: 80940, August 1996.

[25] LOGAN, D.L. A First Course in the Finite Element Method. Boston, USA. PWS-Kent Publishing Company, 1986. 616p.

[26] NASCIMENTO, C.L. Estudo Comparativo de Métodos de Combate à Divergência de Filtros de Kalman. 1988. 248p. Tese (Doutorado) - ITA. S.J. dos Campos.

[27] PAULSON, K.; BRECKON, W.; PIDCOCK, M. A Hybrid Phantom for Impedance tomography. Institute of Physical Sciences in Medicine Clin. Phys. Physiol. Meas., v.13, Suppl. A, p.155-159, 1992.

[28] PHILLIPS, C.J. Glass its Industrial Applications. New York: Reinhold Publishing Corporation, 1960. 252p.

[29] PRESS, W.H. et al. Numerical Recipes in C. The Art of Scientific Computing. Second Edition. New York: Cambridge University Press, 1992. 994p.

[30] SMITH, R.W.M.; FREESTON, I.L.; BROWN, B.H. A Real-Time Electrical Impedance Tomography System for Clinical Use-Desing and Preliminary Results. IEEE Transactions on Biomedical Engineering, v.42, n.2, p.133-140, February 1995. 
[31] SORENSON, H.W. Introduction in Kalman Filtering : Theory and Application. IEEE, N. York, p.118, 1985.

[32] TRIGO, C.F. Filtro Estendido de Kalman Aplicado à Tomografia por Impedância Elétrica. 2001. 98p. Dissertação (Mestrado) - Escola Politécnica, Universidade de São Paulo. São Paulo.

[33] UNIVERSIDADE FEDERAL DO RIO GRANDE DO SUL. Abril 2002. A Descoberta dos Raios X. Disponível em : <http:// www.if.ufrgs.br/tex/fis142/raiosx/rxhist.html > . Acesso em : 14 de maio. 2002.

[34] VANEGAS, N.A. Diseño y Construcción de un Equipo Móvil de Rayos X. 1992, 144p, Tesis de grado - Departamento de Ingeniería Mecánica, Universidad Nacional de Colombia. Colombia

[35] VAUHKONEN, M. Electrical Impedance Tomography and Prior Information. 1997. 110p. Tese (Duotorado) - University of Kuopio, Kuopio. Finland.

[36] VAUHKONEN, M.; KARJALAINEN, P.A.; KAIPIO, J.P. A Kalman Filter Approach to Track Fast Impedance Changes in Electrical Impedance Tomography. IEEE Transactions on Biomedical Engineering, v.45, n.4, p.486-493, April 1998.

[37] WATKINS, D.S. Fundamentals of Matrix Computations. New York, John Wiley \& Sons, 1991. 449p.

[38] WEBSTER, J.C.; HENDERSON, R.P. An Impedance Camera for Spatially Specific Measurements of the Torax. IEEE Transactions on Biomedical Enginnering, v.25, p.250-254, 1978.

[39] YORKEY, T.J.; WEBSTER, J.G.; TOMPKINS, W.J. Comparing Reconstruction Algorithms for Electrical Impedance Tomography. IEEE Transactions on Biomedical Engineering, v.BME-34, n.11, p.843-852, November 1987. 


\section{Apêndice A}

\section{FORMULAÇÃO DO FILTRO DE KALMAN}

Apresentam-se, nestas seções, resultados referentes ao método do Filtro de Kalman discreto. O desenvolvimento apresentado a seguir baseia-se nos trabalhos de Kalman [22, 23] e Brown [8], dos quais são derivadas as equações recursivas do filtro.

Seja o processo estocástico a ser estimado, modelado na seguinte forma :

$$
x_{k+1}=\Phi_{k} x_{k}+w_{k} .
$$

Assume-se que a observação (ou medida) ocorre em pontos discretos do tempo, de acordo com a relação linear :

$$
z_{k}=H_{k} x_{k}+v_{k}
$$

onde

$x_{k\left(n_{x} 1\right)}:$ vetor de estado no tempo $t_{k}$

$\Phi_{k\left(n_{x} n\right)}$ : matriz relacionando $x_{k}$ a $x_{k+1}$ na ausência de função forçante. Se $x_{k}$ for uma amostra do processo contínuo, $\Phi_{k}$ será a matriz de transição usual do estado;

$w_{k\left(n_{x} 1\right)}$ : seqüência de ruído branco (não correlacionada), com estrutura de covariância conhecida;

$z_{k\left(m_{x} 1\right)}:$ vetor de medidas no tempo $t_{k}$;

$H_{k\left(m_{x} n\right)}$ : matriz que fornece a conexão ideal (sem ruído) entre as medidas e o vetor de estado no tempo $t_{k}$;

$v_{k\left(m_{x} 1\right)}$ : vetor do erro de medida, assume-se que é uma seqüência de ruído branco com estrutura de covariância conhecida e não correlacionada com a sequiência $w_{k}$; 
$m, n>=1:$ inteiros.

As matrizes de covariância para os vetores $w_{k}$ e $v_{k}$ são dadas por :

$$
\begin{aligned}
& E\left(w_{k} w_{j}^{t}\right)=\left\{\begin{array}{l}
Q_{k}, j=k \\
0, j \neq k
\end{array}\right. \\
& E\left(v_{k} v_{j}^{t}\right)=\left\{\begin{array}{l}
R_{k}, j=k \\
0, j \neq k
\end{array}\right.
\end{aligned}
$$

$E\left(w_{k} v_{j}^{t}\right)=0$ para todo $j$ e $k$, onde o expoente $t$ denota o transposto do vetor.

Supõe-se que há uma estimativa inicial do processo em algum ponto no tempo $t_{k}$, a qual esta baseada no conhecimento do processo anterior a $t_{k}$. Esta estimativa é denotada por $\hat{x}_{k}^{(-)}$. Supõese, também, conhecida a matriz de covariância do erro associado a $\hat{x}_{k}^{(-)}$. Isto é, definindo-se o erro da estimação como $e_{k}^{(-)}=x_{k}-\hat{x}_{k}^{(-)}$, e assumindo que sua média é zero, a matriz associada de covariância do erro é dada por :

$$
P_{k}^{(-)}=E\left(e_{k}^{(-)} e_{k}^{(-) t}\right)=E\left(\left(x_{k}-\hat{x}_{k}^{(-)}\right)\left(x_{k}-\hat{x}_{k}^{(-)}\right)^{t}\right) .
$$

Em muitos casos, inicia-se o problema de estimação sem nenhuma medida a priori. Neste caso, se a média do processo for zero, a estimativa inicial é zero e a matriz de covariância do erro associado é justamente a matriz de covariância de $x$ por si mesma.

Com a suposição de uma estimativa inicial $\hat{x}_{k}^{(-)}$, pode-se usar a medida $z_{k}$ para melhorar a estimativa anterior. Assim, escolhe-se uma combinação linear do ruído de medida e da estimativa anterior de acordo com a equação :

$$
\hat{x}_{k}=\hat{x}_{k}^{(-)}+K_{k}\left(z_{k}-H_{k} \hat{x}_{k}^{(-)}\right)
$$

onde

$\hat{x}_{k}$ : é a estimativa atualizada;

$K_{k}$ : é o fator de combinação (ainda não determinado).

O problema consiste em encontrar um fator particular de combinação que produz uma estimativa atualizada que seja ótima em algum sentido. Utiliza-se o método de mínimos quadrados como critério de desempenho.

Forma-se inicialmente a expressão para a matriz de covariância do erro associada com a estimativa atualizada (a posteriori) :

$$
P_{k}=E\left(e_{k} e_{k}^{t}\right)=E\left(\left(x_{k}-\hat{x}_{k}\right)\left(x_{k}-\hat{x}_{k}^{t}\right) .\right.
$$

Substituindo a equação A.4 na equação A.5, tem-se :

$$
P_{k}=E\left\{\left[x_{k}-\left[\hat{x}_{k}^{(-)}+K_{k}\left(z_{k}-H_{k} \hat{x}_{k}^{(-)}\right)\right]\right]\left[x_{k}-\left[\hat{x}_{k}^{(-)}+K_{k}\left(z_{k}-H_{k} \hat{x}_{k}^{(-)}\right)\right]\right]^{t}\right\},
$$


e rearranjando os termos, resulta

$P_{k}=E\left\{\left[\left(x_{k}-\hat{x}_{k}^{(-)}\right)-K_{k}\left(z_{k}-H_{k} \hat{x}_{k}^{(-)}\right)\right]\left[\left(x_{k}-\hat{x}_{k}^{(-)}\right)-K_{k}\left(z_{k}-H_{k} \hat{x}_{k}^{(-)}\right)\right]^{t}\right\}$.

Substituindo nesta última expressão a equação A.2 :

$P_{k}=E\left\{\left[\left(x_{k}-\hat{x}_{k}^{(-)}\right)-K_{k}\left(H_{k} x_{k}+v_{k}-H_{k} \hat{x}_{k}^{(-)}\right)\right]\left[\left(x_{k}-\hat{x}_{k}^{(-)}\right)-K_{k}\left(H_{k} x_{k}+v_{k}-H_{k} \hat{x}_{k}^{(-)}\right)\right]^{t}\right\}$

Aplicando o operador esperança e notando que $\left(x_{k}-\hat{x}_{k}^{(-)}\right)$é o erro de estimação a-priori que é não correlacionado com o erro de medida $v_{k}$, tem-se :

$$
P_{k}=\left(I-K_{k} H_{k}\right) P_{k}^{(-)}\left(I-K_{k} H_{k}\right)^{t}+K_{k} R_{k} K_{k}^{t}
$$

que é a expressão geral para a matriz de covariância do erro, atualizada e aplicada para qualquer ganho $K_{k}$, sendo $I$ a matriz identidade.

Deseja-se encontrar o $K_{k}$ particular que minimiza os termos individuais ao longo da diagonal principal da matriz de covariância, porque estes termos representam as variâncias dos erros de estimação para os elementos do vector de estado que estão sendo estimados. Reagrupando A.7, tem-se :

$$
P_{k}=P_{k}^{(-)}-K_{k} H_{k} P_{K}^{(-)}-P_{k}^{(-)} H_{k}^{t} K_{k}^{t}+K_{k}\left(H_{k} P_{k}^{(-)} H_{k}^{t}+R_{k}\right) K_{k}^{t} .
$$

Havendo termos lineares e quadráticos em $K$, a matriz $P$ pode ser vista como quadrática em $K$, e deseja-se construir uma matriz equivalente "elevando ao quadrado". Asume-se, inicialmente, que a matriz $\left(H_{k} P_{k}^{(-)} H^{t}+R_{k}\right)$ seja simétrica e positiva definida. Escrevendo este termo na forma fatorada $S_{k} S_{k}^{t}$ como :

$$
S_{k} S_{k}^{t}=H_{k} P_{k}^{(-)} H_{k}^{t}+R_{k}
$$

Substituindo a equação A.9 na equação A.8, obtém-se :

$$
P_{k}=P_{k}^{(-)}-K_{k} H_{k} P_{k}^{(-)}-P_{k}^{(-)} H_{k}^{t} K_{k}^{t}+K_{k} S_{k} S_{k}^{t} K_{k}^{t}
$$

Elevando ao quadrado, $P$ pode ser escrita na forma :

$$
P_{k}=P_{k}^{(-)}+\left(K_{k} S_{k}-A_{k}\right)\left(K_{k} S_{k}-A_{k}\right)^{t}-A_{k} A_{k}^{t}
$$

onde $A$ não envolve $K$.

Expandindo a equação A.11 e comparando-a termo a termo com a equação A.10 : 


$$
K_{k} S_{k} A_{k}^{t}+A_{k} S_{k}^{t} K_{k}^{t}=K_{k} H_{k} P_{k}^{(-)}+P_{k}^{(-)} H_{k}^{t} K_{k}^{t}
$$

Facilmente verifica-se que, se

$$
A_{k}=P_{k}^{(-)} H_{k}^{t}\left(S_{k}^{t}\right)^{-1}
$$

a equação A.12 é satisfeita e a equação A.11 é equivalente à equação A.10.

Observa-se que o $1^{o}$ e o $3^{o}$ termo da equação A.11 não envolvem $K_{k}$. Porém o $2^{o}$ termo o envolve, o qual é o produto de uma matriz e sua transposta o que assegura que todos os termos ao longo do eixo principal são não negativos. Deseja-se minimizar os termos diagonais de $P_{k}$. Assim, deve-se ajustar $K_{k}$ de forma que o $2^{o}$ termo da equação A.11 seja zero. Escolhe-se, então, $K_{k}$ tal que $K_{k} S_{k}=A_{k}$, ou, usando as equações A.9 e A.13 :

$$
K_{k}=A_{k} S_{k}^{-1}=P_{k}^{(-)} H_{k}^{t}\left(S_{k} S_{k}^{t}\right)^{-1}=P_{k}^{(-)} H_{k}^{t}\left(H_{k} P_{k}^{(-)} H_{k}^{t}+R_{k}\right)^{-1} .
$$

Este $K_{k}$ particular que minimiza a estimação do erro quadrático médio, é chamado Ganho de Kalman.

A matriz de covariância associada com a estimativa ótima pode agora ser computada. Da equação A.8, temos :

$$
P_{k}=P_{k}^{(-)}-K_{k} H_{k} P_{k}^{(-)}-P_{k}^{(-)} H_{k}^{t} K_{k}^{t}+K_{k}\left(H_{k} P_{k}^{(-)} H_{k}^{t}+R_{k}\right) K_{k}^{t}
$$

Substituindo a equação A.14 na equação A.15, resulta :

$$
P_{k}=P_{k}^{(-)}-K_{k} H_{k} P_{k}^{(-)}-P_{k}^{(-)} H_{k}^{t} K_{k}^{t}+P_{k}^{(-)} H_{k}^{t} K_{k}^{t} \text {. }
$$

Cancelando os dois últimos termos, a equação fica

$$
P_{k}=P_{k}^{(-)}-K_{k} H_{k} P_{k}^{(-)} \text {então }
$$

$$
P_{k}=\left(I-K_{k} H_{k}\right) P_{k}^{(-)} .
$$

Nota-se que a equação A.15 é válida para qualquer ganho, ótimo ou não, enquanto que a equação A.16 é válida somente para o ganho ótimo.

Pode-se calcular a medida em $t_{k}$ através do uso da equação A.4 com $K_{k}$ igual ao ganho de Kalman como dado pela equação A.14. Necessita-se de $\hat{x}_{k}^{(-)}$e $P_{k}^{(-)}$e pode-se antecipar uma necessidade semelhante ao passo seguinte para tornar ótima a medida $z_{k+1}$. A estimativa atualizada $\hat{x}_{k}$ é facilmente projetada via matriz de transição. Ignora-se, neste ponto, a contribuição de $w_{k}$ 
na equação A.1 porque sua média é zero e ele é não correlacionado com os $w^{\prime} s$ anteriores. Desta forma,

$$
\hat{x}_{k+1}^{(-)}=\Phi_{k} \hat{x}_{k}
$$

A matriz de covariância do erro associado com $\hat{x}_{k+1}^{(-)}$é obtida inicialmente construindo a expressão para o erro a priori :

$$
P_{k+1}^{(-)}=E\left[e_{k+1}^{(-)}\left(e_{k+1}^{(-)}\right)^{t}\right]=E\left[\left(\Phi_{k} e_{k}+w_{k}\right)\left(\Phi_{k} e_{k}+w_{k}\right)^{t}\right]=\Phi_{k} P_{k} \Phi_{k}^{t}+Q_{k}
$$

Obtêm-se, assim, as quantidades necessárias no tempo $t_{k+1}$ e a medida $z_{k+1}$ pode ser obtida. Resumindo, tem-se o seguinte conjunto de equações recursivas :

- equações de propagação :

propagação de estado :

$$
\hat{x}_{k+1}^{(-)}=\Phi_{k} \hat{x}_{k}
$$

propagação da covariância de estado :

$$
P_{k+1}^{(-)}=\Phi_{k} P_{k} \Phi_{k}^{t}+Q_{k}
$$

- equações de atualização :

ganho do filtro :

$$
K_{k}=P_{k}^{(-)} H_{k}^{t}\left(H_{k} P_{k}^{(-)} H_{k}^{t}+R_{k}\right)^{-1}
$$

atualização da matriz de covariância :

$$
P_{k}=\left(I-K_{k} H_{k}\right) P_{k}^{(-)}
$$

atualização do estado :

$$
\hat{x}_{k}=\hat{x}_{k}^{(-)}+K_{k}\left(z_{k}-H_{k} \hat{x}_{k}^{(-)}\right)
$$

As equações A.19, A.20, A.21, A.22 e A.23 sumarizam o que é conhecido como Filtro de Kalman discreto.

A Figura A.1, apresenta as equações pertinentes e a seqüência de passos computacionais para a aplicação do Filtro de Kalman. 


\section{Filtro de Kalman}

Entra com a estimativa inicial $\hat{x}_{k}^{(-)}$e a covariância do erro $P_{k}^{(-)}$

$\Downarrow$

Cálculo do ganho de Kalman : $K_{k}=P_{k}^{(-)} H_{k}^{t}\left(H_{k} P_{k}^{(-)} H_{k}^{t}+R_{k}\right)^{-1}$

$\Downarrow$

Atualiza estimativa com medida $z_{k}: \hat{x}_{k}=\hat{x}_{k}^{(-)}+K_{k}\left(z_{k}-H_{k} \hat{x}_{k}^{(-)}\right)$

$\Downarrow$

Cálculo da covariância de erro para estimativa atualizada :

$$
\begin{gathered}
P_{k}=\left(I-K_{k} H_{k}\right) P_{k}^{(-)} \\
\Downarrow \\
\text { Projeto adiante }: \hat{x}_{k+1}^{(-)}=\Phi_{k} \hat{x}_{k} \\
\Downarrow \\
\text { Projeto adiante }: P_{k+1}^{(-)}=\Phi_{k} P_{k} \Phi_{k}^{t}+Q_{k}
\end{gathered}
$$

Figura A.1: Esquema do Filtro de Kalman [8]. 


\section{Apêndice B}

\section{ALGORITMO PARA A APLICAÇÃO DO REFINAMENTO ITERATIVO}

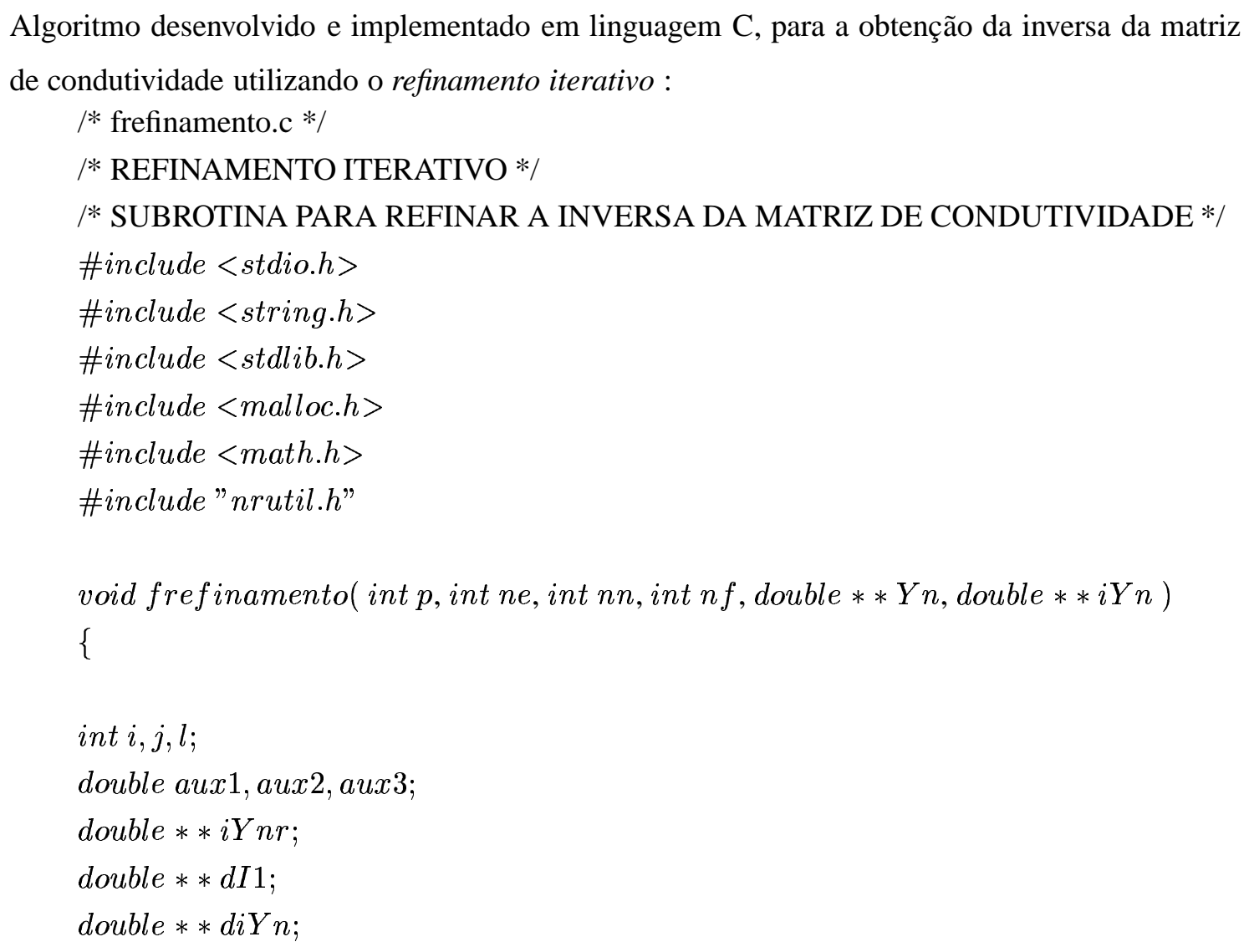




\section{$F I L E * f p$}

$i Y n r=d m a t r i x(1, n n, 1, n n)$
$d I 1=d m a t r i x(1, n n, 1, n n) ;$
$d i Y n=d m a t r i x(1, n n, 1, n n)$

/* Aplicação da equação */

$/ * \delta I_{c i}=Y .\left(i Y_{c i}+\delta i Y_{c i}\right)-I_{c i} * /$

for $(l=1 ; l<=n n ; l++)\{$

for $(i=1 ; i<=n n ; i++)\{$

aux $1=0.0$;

for $(j=1 ; j<=n n ; j++)\{$

aux $1=\operatorname{aux} 1+Y n[i][j] * i Y n[j][l] ;$

\}

$d I 1[i][l]=$ aux 1

\}

$d I 1[l][l]=d I 1[l][l]-1.0 ;$

/* Aplicação da equação */

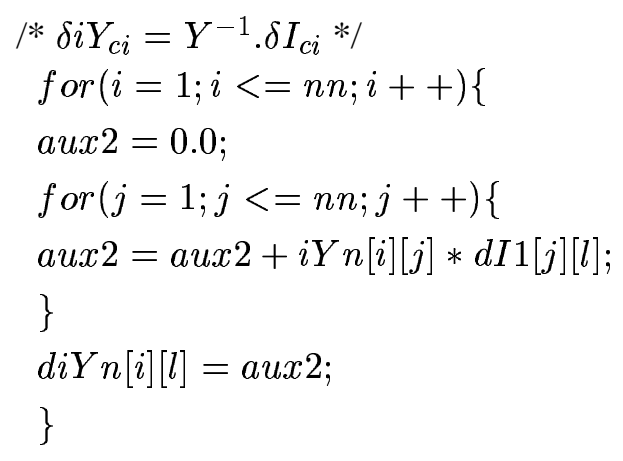

/* Nova solução estimada melhorada */ $/ * i Y r_{c i}=i Y_{c i}-\delta i Y_{c i} * /$ 


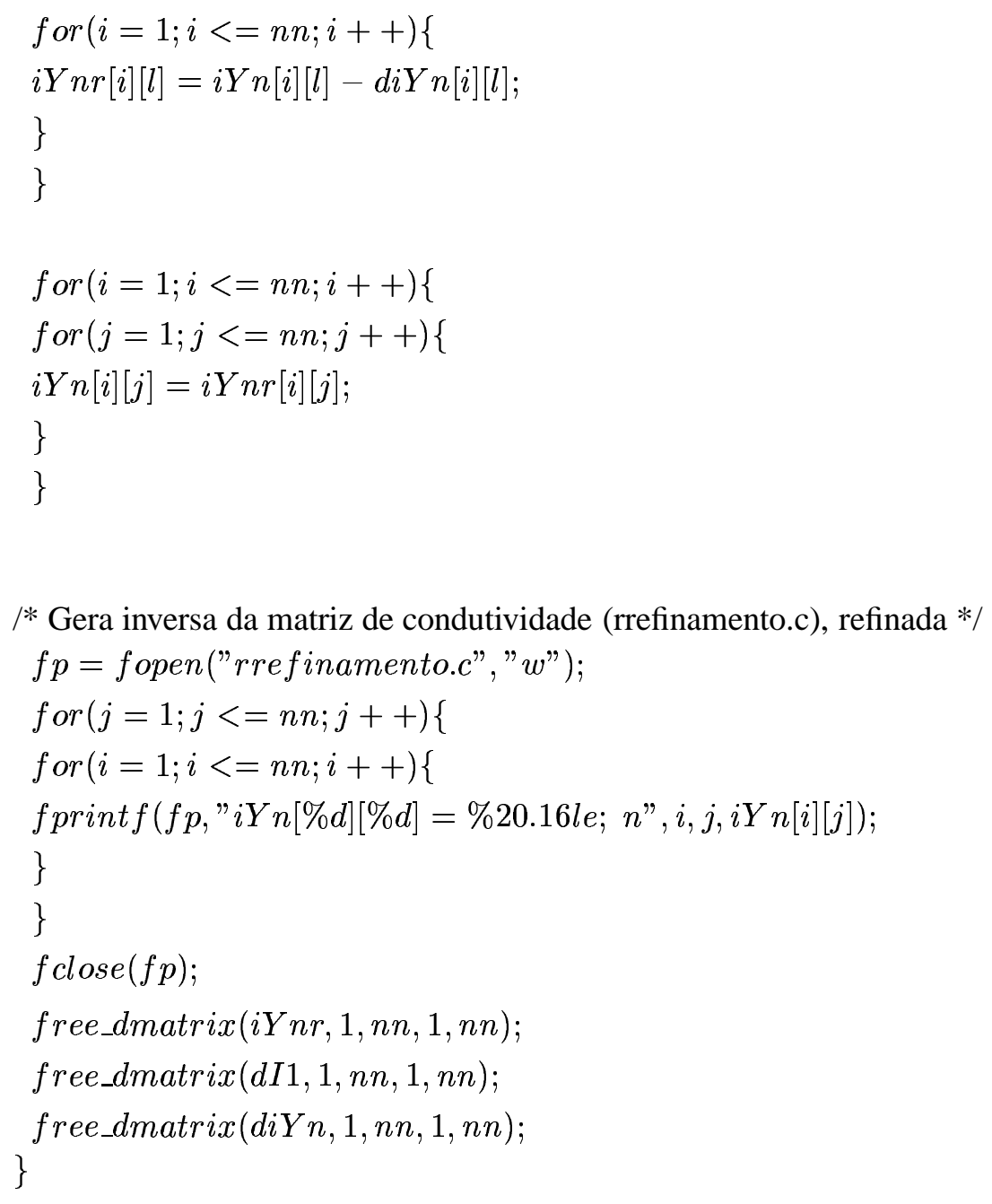




\section{Apêndice C}

\section{ALGORITMO IMPLEMENTADO PARA TOMOGRAFIA POR IMPEDÂNCIA ELÉTRICA}

Apresenta-se a continuação os algoritmos de estimação desenvolvidos para serem aplicados na TIE, os quais foram escritos inicialmente com o software Octave e posteriormente em linguagem C.

\section{C.1 Algoritmo Inicial}

Pela facilidade e rapidez que apresenta o Octave na programação e implementação de algoritmos, foi primeiro escrito e desenvolvido um algoritmo de estimação nesta linguagem, o qual auxiliou na implementação posterior do algoritmo em linguagem $\mathrm{C}$.

O primeiro programa desenvolvido no Octave foi para a solução do problema direto, o qual permite obter a matriz de condutividade e as voltagens dos nós de qualquer malha de elementos finitos, conhecida a matriz de coordenadas $(x, y)$ de cada um dos nós e a matriz de topologia contendo os nós de cada elemento da malha de elementos finitos.

Numa segunda etapa implementou-se neste algoritmo as malhas renumeradas, o qual estimou o tipo de matriz de condutividade obtido e identificou a matriz de condutividade diagonal de banda para posteriormente conformá-la em forma compacta.

Finalmente implementou-se um algoritmo da metodologia para a solução do problema inverso, 
aplicando o Filtro de Kalman, obtendo-se as estimativas da distribuição de condutividades para os elementos da malha de elementos finitos.

Segundo o tamanho das malhas de elementos finitos implementadas, o algoritmo apresentava um determinado tempo de estimação, sendo que para as malhas de 182 elementos, 156 nós e 32 eletrodos o tempo para a estimação da distribuição de condutividades foi da ordem de 8 horas.

Este algoritmo de estimação inicial implementado para a solução do problema existente na TIE, permitiu compreender o comportamento das variáveis e implementar de forma prática as diferentes mudanças que se apresentam no desenvolvimento de um algoritmo deste tipo. Além de ser o algoritmo de comparação ou referência para o algoritmo em linguagem C. Todos os resultados das estimativas de condutividades obtidos em linguagem $\mathrm{C}$ foram avaliados e comparados com este algoritmo inicial apresentando-se resultados com mínimas diferenças numéricas.

\section{C.2 Algoritmo em Linguagem C}

Apresenta-se a continuação na forma detalhada, de cada uma das etapas envolvidas no desenvolvimento do algoritmo em linguagem $\mathrm{C}$, definindo as diferentes subrotinas implementadas para assim facilitar a metodologia utilizada neste algoritmo de estimação:

1. desenho da malha : malha discretizada que representa uma seção transversal do tórax, contendo ne elementos (triangulares e retangulares), o $n n$ nós de cada elemento da malha onde os $n f$ últimos elementos são os eletrodos. Para gerar esta malha de elementos finitos utilizou-se o software FELt, módulo Corduroy;

2. cd.dat : matriz com as coordenadas $x_{i}$ e $y_{i}, \operatorname{com} i=1, . .$, ne de cada um dos nós da malha de elementos finitos, obtida com o software FELt;

3. nodes.dat : matriz com as coordenadas dos três nós de cada um dos elementos planos triangulares e dos quatro nós de cada um dos elementos retangulares que representam os eletrodos. E também conhecida como matriz de topologia;

4. tomo.c : subrotina para o cálculo da matriz de condutividade simbólica, para o cálculo desta precisa-se conhecer $n e$, número de elementos da malha, $n n$ número de nós da malha, $n f$ número de eletrodos, a espessura da malha, a metade da largura dos eletrodos e as matrizes cd.dat e nodes.dat, gerando os seguintes arquivos de dados :

- condutividade.c : matriz de condutividade $Y$ simbólica, matriz em função das condutividades dos elementos, com dimensão de $n n_{x} n n$;

- dydrok.c : matriz de derivada com respeito à condutividade de cada matriz de condutividade local para os elementos triangulares da malha; 
- dydrokelet.c : matriz de derivada com respeito à condutividade, de cada matriz de condutividade local para os elementos retangulares da malha;

- linha.c : matriz com o número de cada um dos nós das linhas que correspondem a cada elemento da matriz de condutividade local para os elementos triangulares;

- linhaelet.c : matriz com o número de cada um dos nós das linhas que correspondem a cada elemento da matriz de condutividade local para os elementos retangulares (eletrodos);

- coluna.c : matriz com o número de cada um dos nós das colunas que correspondem a cada elemento da matriz de condutividade local para os elementos triangulares;

- colunaelet.c : matriz com o número de cada um dos nós das colunas que correspondem a cada elemento da matriz de condutividade local para os elementos retangulares (eletrodos);

5. condinicias.c : matriz com dados das estimativas de condutividades iniciais dos elementos da malha;

6. rkp.c : vetor de condutividades iniciais dos elementos por ROIS. Neste trabalho agrupou-se os elementos da malha em 5, 8 e 16 ROIS;

7. dadosexperimentais.c : dados adquiridos experimentalmente, voltagens medidas segundo o tipo de padrão de corrente aplicado;

8. correntes.c : matriz de correntes aplicadas nos eletrodos;

9. Pkp.c : condição inicial da matriz de covariância do erro de estimação;

10. MTkn1.c : matriz de transição de estado, identidade;

11. Qkn1.c : matriz de covariância do ruído no estado, é diagonal com elementos iguais;

12. Rk.c : matriz de covariância do ruído na medida;

13. I.c : matriz de identidade;

14. fcontornoY.c : subrotina para as condições de contorno da matriz de condutividade $Y$, segundo o tipo de padrão de corrente $t p c$, calcula a inversa da matriz de condutividade e as voltagens dos nós;

15. fsensibilidade.c : subrotina para calcular a matriz de sensibilidade $H$ para todos os elementos;

16. fHreducida.c : subrotina para obter a matriz de sensibilidade reduzida $H$, segundo as ROIS. Consiste em somar as colunas que correspondem aos elementos de cada região de interesse;

17. fFiltroKalman.c : subrotina para a aplicação do Filtro de Kalman e estimar as novas condutividades por ROIS;

18. fcondutividadesElem.c : subrotina para fornecer as condutividades dos elementos a partir das condutividades obtidas por ROIS; 
19. fnrkp.c : subrotina para calcular o novo vetor de condutividades por ROIS a partir das últimas estimativas de condutividades obtidas pelo Filtro de Kalman para os elementos da malha;

20. fcentrogravedade.c : subrotina para o cálculo do centro de gravidade de cada um dos elementos da malha;

21. fdadosgrafico2dROIS.c : subrotina para gerar os dados para o gráfico em 2 dimensões das condutividades de cada região de interesse ROIS no tempo, evolução temporal das condutividades;

22. fdadosgraficos3dCI.c : subrotina para gerar a matriz com os dados das condutividades xya.dat e impedâncias xyai.dat, para os gráficos em 3 dimensões;

23. xya.dat : arquivo com dados do centro de gravidade de cada elemento $(x c, y c)$ e a condutividade referente ao elemento;

24. xyai.dat : arquivo com dados do centro de gravidade de cada elemento $(x c, y c)$ e a impedância referente ao elemento;

25. gcondutividadesROIS.gnu : gráfico em duas dimensões da evolução temporal das condutividades por ROIS utilizando o software gnuplot;

26. grafico3d.gnu : gráfico em três dimensões das condutividades dos elementos utilizando o software gnuplot a partir dos dados xya.dat;

27. grafico3di.gnu : gráfico em três dimensões das impedâncias dos elementos utilizando o software gnuplot a partir dos dados xyai.dat.

Foram usadas rotinas auxiliares nos diferentes cálculos e que estão disponíveis no Numerical Recipes in $C$ [29].

\section{C.2.1 Fluxograma}

Apresenta-se a continuação do fluxograma que determina a ordem lógica do algoritmo para a estimação de condutividades na TIE, aplicando o Filtro de Kalman.

onde

$n n$ : número de nós da malha de elementos finitos;

ne : número de elementos da malha de elementos finitos;

$n f$ : número de eletrodos, os últimos estudos têm-se desenvolvidos com um arranjo de 32 eletrodos;

$n m$ : número de escalares na matriz de condutividade local, dos elementos triangulares;

nme : número de escalares na matriz de condutividade local dos elementos retangulares (eletrodos);

$R O I S$ : número de regiões de interesse; 


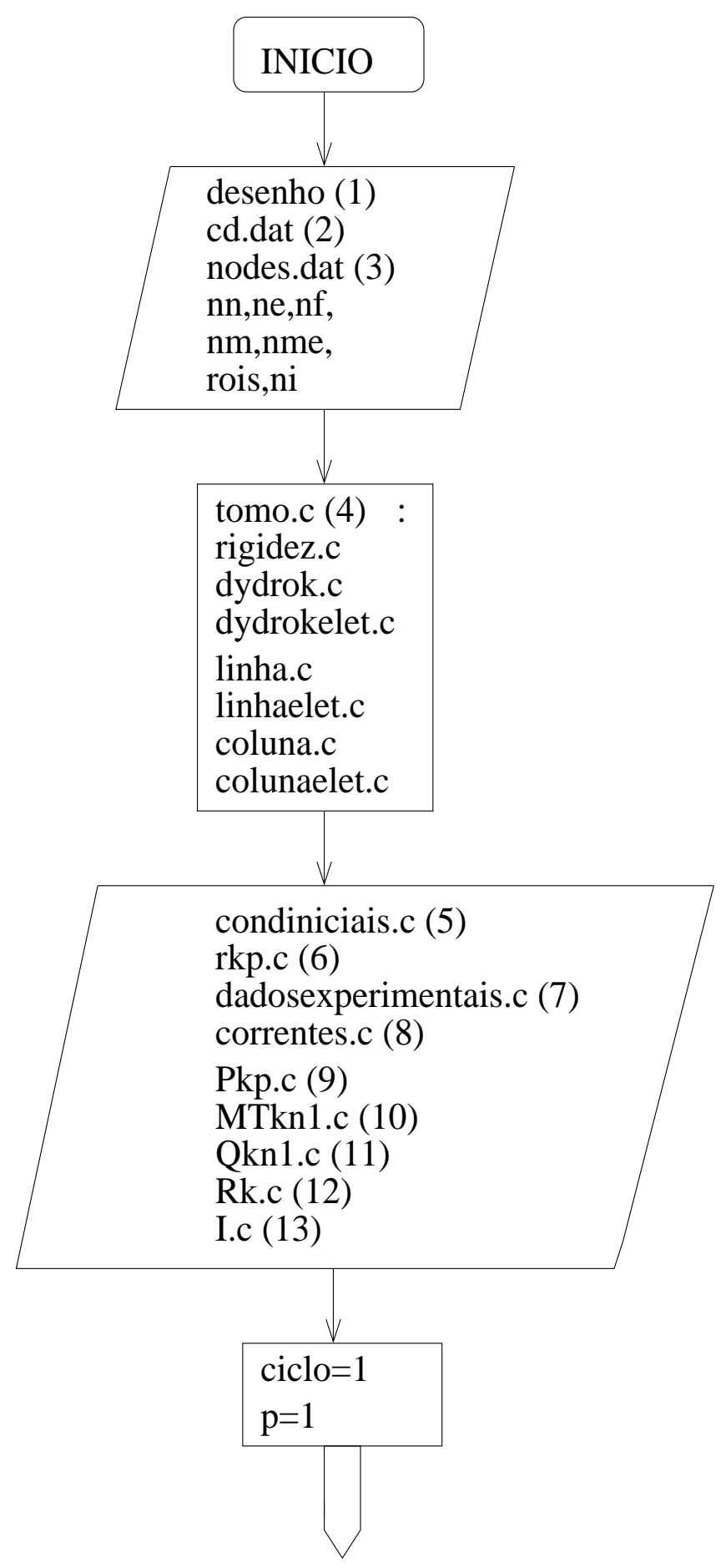

Figura C.1: Fluxograma, parte 1. 


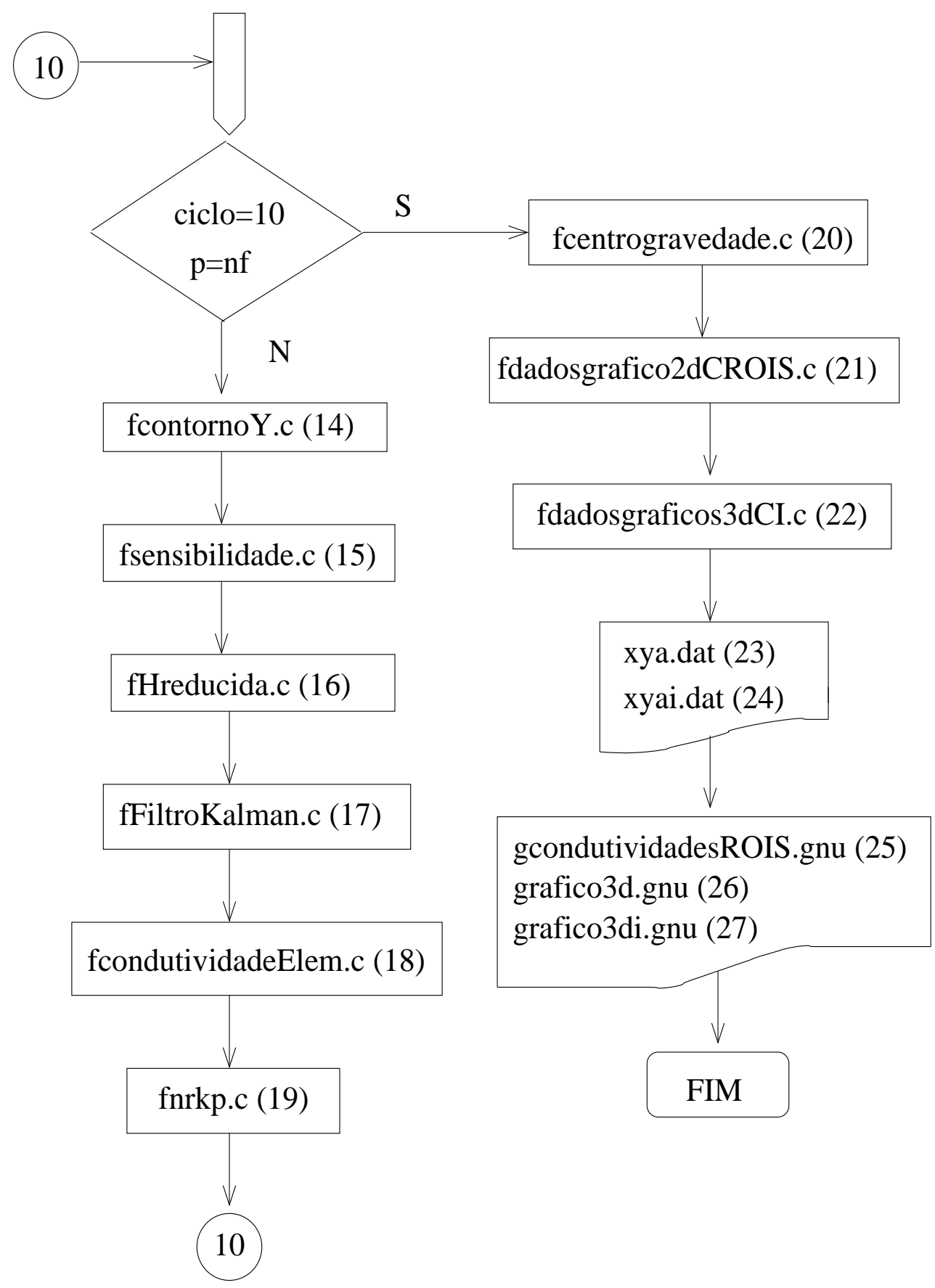

Figura C.2: Continuação do Fluxograma. 
tpc : tipo de padrão de corrente aplicado;

$n i$ : número de iterações para a convergência do algoritmo.

O fluxograma anterior complementa e facilita a descrição dos algoritmos desenvolvidos. Observase pelo fluxograma os números que correspondem aos detalhes de cada etapa ou subrotina específica.

\section{C.2.2 Algoritmo Principal Desenvolvido}

Apresenta-se a continuação o algoritmo principal desenvolvido em linguagem C, para a obtenção das estimativas da distribuição de impedâncias de um objeto ou um corpo, neste caso o tórax humano, para malhas renumeradas e com refinamento iterativo.

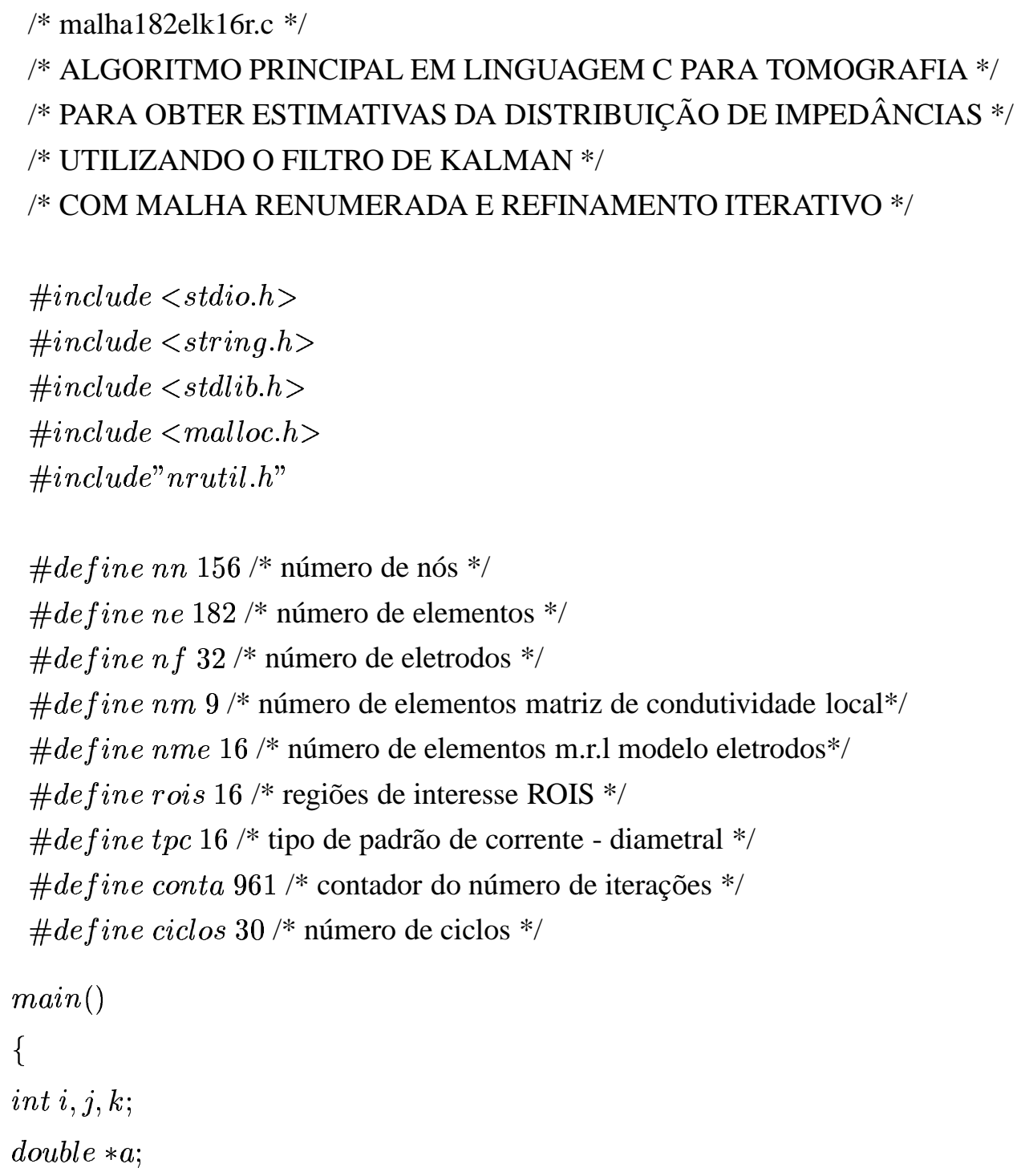




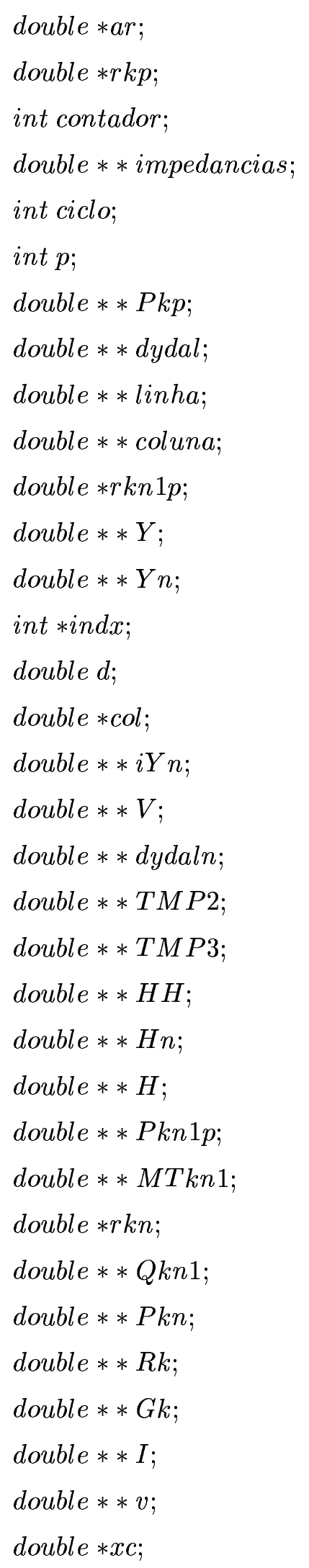


double $* y c$;

double *ai;

double *normaGk;

double *residuo;

double $* * C$;

double aux 3 ;

$F I L E * f p$

$a=$ dvector $(1, n e)$;

$a r=$ dvector $(1$, rois $)$;

$r k p=$ dvector $(1$, rois $)$;

impedancias $=$ dmatrix $(1$, rois, 1, conta $)$;

$P k p=$ dmatrix $(1$, rois, 1, rois $)$;

$d y d a l=d m a t r i x(1, n e, 1, n m e) ;$

linha $=$ dmatrix $(1$, ne $, 1, n m e)$;

coluna $=$ dmatrix $(1$, ne, 1, nme $)$;

$r k n 1 p=$ dvector $(1$, rois $)$;

$Y=$ dmatrix $(1, n n, 1, n n)$;

$Y n=\operatorname{dmatrix}(1, n n, 1, n n)$;

ind $x=$ ivector $(1, n n)$;

col $=$ dvector $(1, n n)$;

$i Y n=\operatorname{dmatrix}(1, n n, 1, n n)$;

$V=$ dmatrix $(1, n n, 1, n f)$;

dydaln $=$ dmatrix $(1, n n, 1, n n)$;

$T M P 2=$ dmatrix $(1, n n, 1, n e) ;$

$T M P 3=$ dmatrix $(1, n n, 1, n e)$;

$H H=$ dmatrix $(1, n f, 1, n e)$;

$H n=$ dmatrix $(1, n f, 1$, rois $)$;

$H=$ dmatrix $(1, n f, 1$, rois $)$;

Pkn1p = dmatrix $(1$, rois, 1, rois $)$;

MTkn1 = dmatrix $(1$, rois, 1, rois $)$;

$r k n=\operatorname{dvector}(1$, rois $)$;

Qkn1 $=$ dmatrix $(1$, rois, 1, rois $)$; 


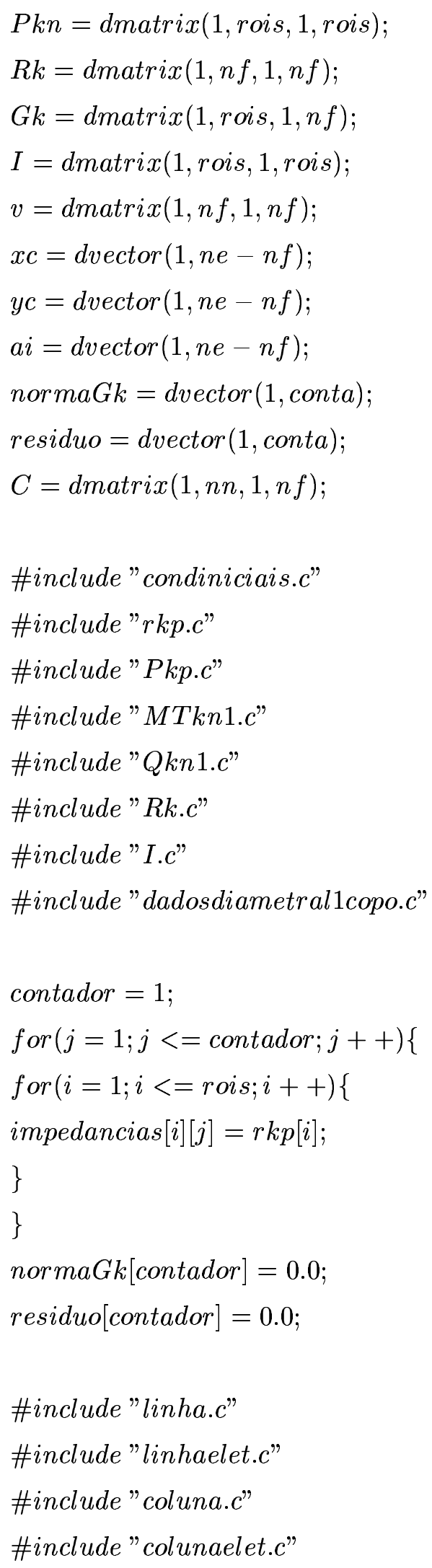




\section{\#include "correntes.c"}

/* Ciclo de iterações */

for $($ ciclo $=1 ;$ ciclo $<=$ ciclos $;$ ciclo ++$)\{$

for $(p=1 ; p<=n f ; p++)\{$

${ }^{*} p=1 ; * /$

/* fnrkp.c */

/* função para calcular */

/* vetor de impedâncias por regiões de interesse $r k p . c * /$

fnrkp (a, ar, rkp, rois $)$;

\#include " condutividade.c"

\#include "dydrok.c"

\#include"dydrokelet.c"

$/ *$ fcontornoY.c */

/* função para as condições de contorno da matriz de condutividade $Y * /$

/* segundo o tipo de padrão de corrente (tpc) */

fcontornoY $(Y, p, t p c, Y n, n e, n n, n f$, rois, ind $x, d$, col, $i Y n)$;

fp = fopen (" condutividaden.c", "w");

for $(j=1 ; j<=n n ; j++)\{$

for $(i=1 ; i<=n n ; i++)\{$

fprintf $(f p, " Y[\% d][\% d]=\% 20.16 l e ; n ", i, j, Y n[i][j])$;

\}

\}

fclose $(f p)$;

/* frefinamento.c */

/* função de refinamento iterativo */

/* para refinar a inversa da matriz de condutividade */

frefinamento $(p, n e, n n, n f, Y n, i Y n)$; 


$$
\begin{aligned}
& \operatorname{for}(i=1 ; i<=n n ; i++)\{ \\
& \operatorname{aux} 3=0.0 \\
& \operatorname{for}(j=1 ; j<=n n ; j++)\{ \\
& \operatorname{aux} 3=a u x 3+i Y n[i][j] * C[j][p] \\
& \} \\
& V[i][p]=\operatorname{aux} 3 \\
& \}
\end{aligned}
$$

/* fsensibilidade.c */

/* função para obter a matriz de sensibilidade $H H *$ /

/* geração da matriz zeros de derivadas dydaln */

/* conformação da matriz dydaln com os dados das */

/* matrizes linha e coluna, para cada elemento */

/* condições de contorno para a matriz dydaln */

$/ *$ segundo o tipo de padrão de corrente $(t p c) * /$

f sensibilidade(ne, nn, nf, dydaln, nm, nme, linha, coluna, dydal, $p$, tpc,

V, TMP2, iYn, TMP3, HH);

/* fHreducida.c */

/* função para calcular */

/* a matriz de sensibilidade reduzida */

/* por regiões de interesse H.c */

fHreducida $(H H, n f, H n, H$, rois, ne $)$;

contador $=$ contador +1 ;

/* fFiltroKalman.c */

/* aplicação do Filtro de Kalman */

/* função para estimar */

/* as impedâncias dos elementos $r k p . c *$ /

fFiltroKalman (rois, rkp, rkn1p, Pkp, Pkn1p, MTkn1, rkn, Qkn1, Pkn, nf, $H, R k, G k, I, v, p, V$, contador, residuo);

$\operatorname{for}(i=1 ; i<=$ rois $; i++)\{$ 
impedancias $[i][$ contador $]=\operatorname{rkp}[i]$

\}

/* fcondutividadesElem.c */

/* função para fornecer */

/* as condutividades e impedâncias dos elementos *

/* a partir das condutividades ou impedâncias por regiões de interesse */

f condutividadesElem(ne, $n f$, rois, $r k p, a)$;

for $(i=1 ; i<=$ rois $; i++)\{$

printf("Impedancias - regioes $[\% d][\% d]=\% 20.16 l e ; n ", i$, contador, impedancias $[i][$ contador $])$;

\}

/* fnormaganho.c */

/* função para graficar o comportamento da norma espectral */

/* da matriz de ganho de Kalman */

fnormaganho(ne, nn, nf, rois, Gk, contador, normaGk);

/* fdadosgrafico2dCROIS.c *//* função que gera os dados *//* para o gráfico em 2D das impedâncias de cada $R O I S *$ /

/* em função do tempo ou de cada iteração */

fdadosgrafico2dCROIS(rois, contador, impedancias);

\}

\}

/* fcentrogravedade.c */

/* função que gera os dados para */

/* o gráfico em 3D das impedâncias */

/* cálculo do centro de gravidade dos elementos */

fcentrogravedade $(n e, n n, n f, x c, y c)$;

/* fdadosgraficos3dCI.c */

/* função que gera as matrizes de dados para */ 
/* os gráficos em 3D das impedâncias xya.dat */

fdadosgraficos $3 d C I(n e, n f, x c, y c, a, a i)$;

free_dvector $(a, 1, n e)$;

free_dvector (ar, 1, rois $)$;

free_dvector $(r k p, 1$, rois $)$;

free_dmatrix(impedancias, 1, rois, 1, conta $)$;

free_dmatrix $(P k p, 1$, rois, 1, rois $)$;

free_dmatrix (dydal, 1, ne, 1, nme);

free_dmatrix(linha, 1, ne, 1, nme);

free_dmatrix (coluna, 1, ne, 1, nme);

free_dvector $(r k n 1 p, 1$, rois $)$;

free_dmatrix $(Y, 1, n n, 1, n n)$;

free_dmatrix $(Y n, 1, n n, 1, n n)$;

free_ivector $($ ind $x, 1, n n)$;

free_dvector $(\mathrm{col}, 1, n n)$;

free_dmatrix $(i Y n, 1, n n, 1, n n)$;

free_dmatrix $(V, 1, n n, 1, n f)$;

free_dmatrix (dydaln, $1, n n, 1, n n)$;

free_dmatrix $(T M P 2,1, n n, 1, n e)$;

free_dmatrix $(T M P 3,1, n n, 1, n e)$;

free_dmatrix $(H H, 1, n f, 1, n e)$;

free_dmatrix $(H n, 1, n f, 1$, rois $)$;

free_dmatrix $(H, 1, n f, 1$, rois $)$;

free_dmatrix (Pkn1p, 1, rois, 1, rois $)$;

free_dmatrix (MTkn1, 1, rois, 1, rois $)$;

free_dvector $(r k n, 1$, rois $)$;

free_dmatrix $(Q k n 1,1$, rois, 1, rois $)$;

free_dmatrix $(P k n, 1$, rois, 1 , rois $)$;

free_dmatrix $(R k, 1, n f, 1, n f)$;

free_dmatrix $(G k, 1$, rois, $1, n f)$;

free_dmatrix $(I, 1$, rois, 1, rois $)$;

free_dmatrix $(v, 1, n f, 1, n f)$;

free_dvector $(x c, 1, n e-n f)$; 
free_dvector $(y c, 1, n e-n f)$;

free_dvector $(a i, 1, n e-n f)$;

free_dvector (normaGk, 1, conta);

free_dvector (residuo, 1, conta);

free_dmatrix $(C, 1, n n, 1, n f)$;

\} 